\title{
Activation pattern and modulation of pain related structures in animal models of migraine
}

\author{
Zsuzsanna Bohár M.Sc. \\ Ph.D. Thesis \\ Doctoral School of Clinical Medicine \\ Department of Neurology \\ Faculty of Medicine \\ Albert Szent-Györgyi Clinical Center \\ University of Szeged
}

Supervisor: Árpád Párdutz M.D., Ph.D.

Szeged

2016 
Original publications directly related to the Ph.D. thesis:

I. Bohár Z, Nagy-Grócz G, Fejes-Szabó A, Tar L, László AM, Büki A, Szabadi N, Vraukó V, Vécsei L, Párdutz Á.

Diverse effects of Brilliant Blue $G$ administration in models of trigeminal activation in the rat. J Neural Transm. 2015 Dec;122(12):1621-31.

IF: 2.402 (2014)

II. Fejes-Szabó A, Bohár Z, Nagy-Grócz G, Vámos E, Tar L, Pődör B, Tajti J, Toldi J, Vécsei L, Párdutz A.

Effect of Probenecid on the Pain-Related Behaviour and Morphological Markers in Orofacial Formalin Test of the Rat.

CNS Neurol Disord Drug Targets 2015;14(3):350-9.

IF: 2.628 (2014)

III. Bohár Z, Fejes-Szabó A, Tar L, Varga H, Tajti J, Párdutz Á, Vécsei L.

Evaluation of c-Fos immunoreactivity in the rat brainstem nuclei relevant in migraine pathogenesis after electrical stimulation of the trigeminal ganglion.

Neurol Sci. 2013 Sep;34(9):1597-604. doi: 10.1007/s10072-013-1292-1.

IF: 1.495

Cumulative impact factor of the publications directly related to the thesis: $\mathbf{6 . 5 2 5}$ 
Publications not directly related to the Ph.D. thesis:

Bohár Z, Párdutz Á, Vécsei L.

Tryptophan Catabolites and Migraine.

Curr Pharm Des. 2016;22(8):1013-21.

IF: 3.452 (2014)

Nagy-Grócz G, Tar L, Bohár Z, Fejes-Szabó A, Laborc KF, Spekker E, Vécsei L, Párdutz Á. The modulatory effect of anandamide on nitroglycerin-induced sensitization in the trigeminal system of the rat.

Cephalalgia 2015 Oct 28. pii: 0333102415613766. [Epub ahead of print]

IF: 4.891 (2014)

Bohár Z, Toldi J, Fülöp F, Vécsei L.

Changing the face of kynurenines and neurotoxicity: therapeutic considerations. Int J Mol Sci. 2015 Apr 29;16(5):9772-93. doi: 10.3390/ijms16059772.

IF: 2.862 (2014)

Tuboly G, Tar L, Bohár Z, Sáfrány-Fárk Á, Petrovszki Z, Kékesi G, Vécsei L, Párdutz Á, Horváth G.

The inimitable kynurenic acid: The roles of different ionotropic receptors in the action of kynurenic acid at a spinal level.

Brain Res Bull. 2015 Mar;112:52-60. doi: 10.1016/j.brainresbull.2015.02.001.

IF: 2.718 (2014)

Fejes-Szabó A, Bohár Z, Vámos E, Nagy-Grócz G, Tar L, Veres G, Zádori D, Szentirmai M, Tajti J, Szatmári I, Fülöp F, Toldi J, Párdutz Á, Vécsei L.

Pre-treatment with new kynurenic acid amide dose-dependently prevents the nitroglycerineinduced neuronal activation and sensitization in cervical part of trigemino-cervical complex. J Neural Transm. 2014 Jul;121(7):725-38. doi: 10.1007/s00702-013-1146-2.

IF: $\mathbf{2 . 4 0 2}$ 
Párdutz A, Fejes A, Bohár Z, Tar L, Toldi J, Vécsei L.

Kynurenines and headache.

J Neural Transm. 2012 Feb;119(2):285-96. doi: 10.1007/s00702-011-0665-y.

IF: 3.052

Tajti J, Párdutz A, Vámos E, Tuka B, Kuris A, Bohár Z, Fejes A, Toldi J, Vécsei L. Migraine is a neuronal disease.

J Neural Transm. 2011 Apr;118(4):511-24. doi: 10.1007/s00702-010-0515-3.

\section{IF: 2.73}

Vámos E, Párdutz A, Varga H, Bohár Z, Tajti J, Fülöp F, Toldi J, Vécsei L.

l-kynurenine combined with probenecid and the novel synthetic kynurenic acid derivative attenuate nitroglycerin-induced nNOS in the rat caudal trigeminal nucleus.

Neuropharmacology 2009 Sep;57(4):425-9. doi: 10.1016/j.neuropharm.2009.06.033.

IF: 3.909

Varga H, Párdutz Á, Vámos E, Bohár Z, Bago F, Tajti J, Bari F, Vécsei L.

Selective inhibition of cyclooxygenase-2 attenuates nitroglycerin-induced calmodulindependent protein kinase II alpha in rat trigeminal nucleus caudalis.

Neurosci Lett. 2009 Feb 20;451(2):170-3. doi: 10.1016/j.neulet.2008.12.038.

IF: 1.925

Cumulative impact factor of publications not directly related to the thesis: $\mathbf{2 7 . 9 4 1}$

Total impact factor: 34.466 


\section{Table of contents}

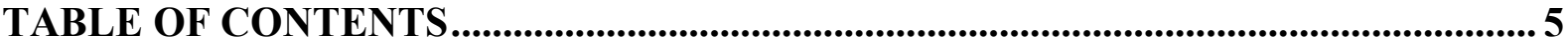

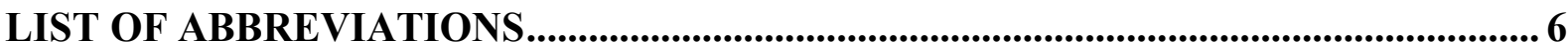

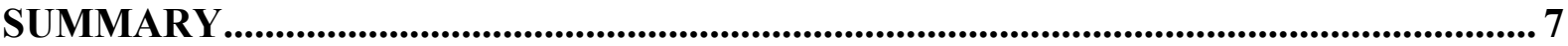

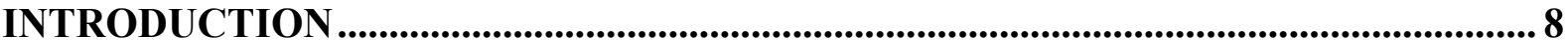

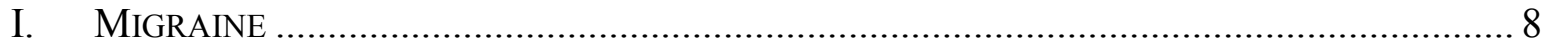

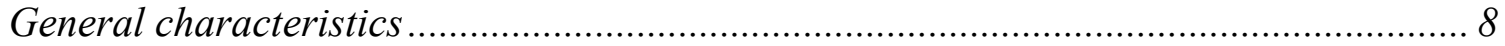

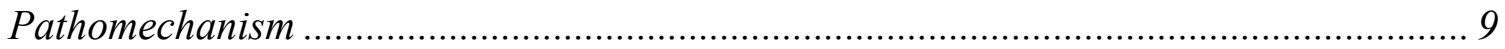

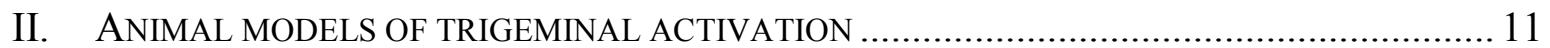

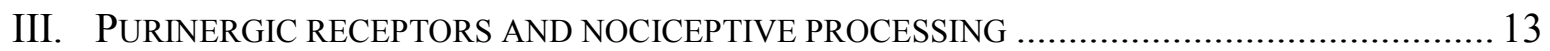

IV. PROBENECID AND NOCICEPTIVE PROCESSING ........................................................... 14

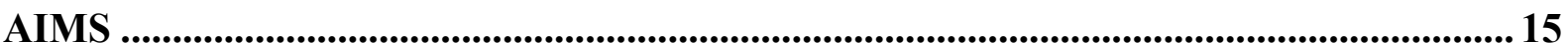

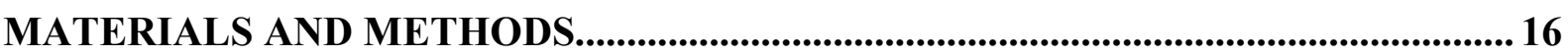

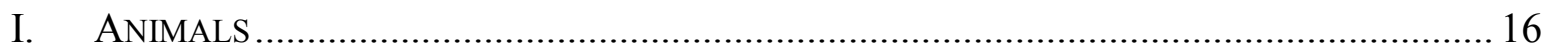

II. ACTIVATION PATTERN AFTER TRIGEMINAL STIMULATION …....................................... 16

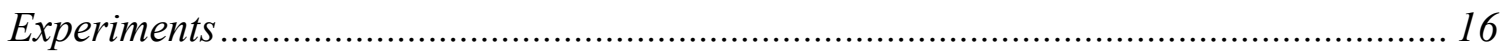

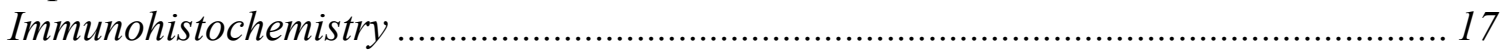

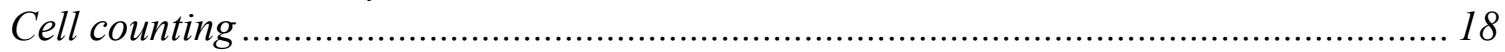

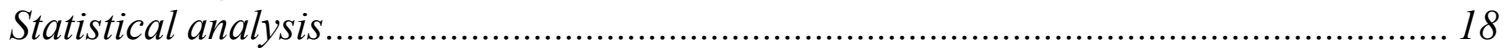

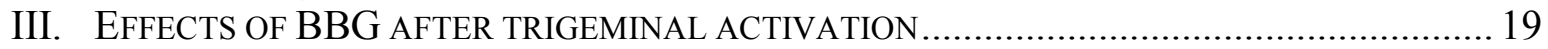

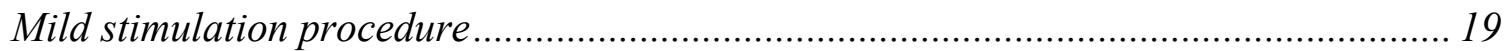

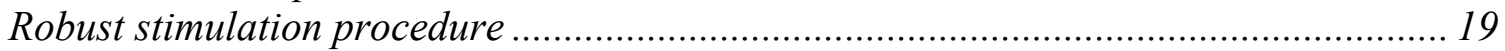

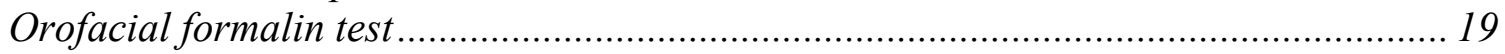

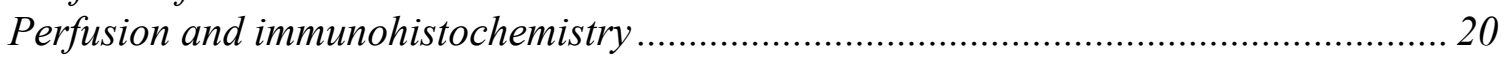

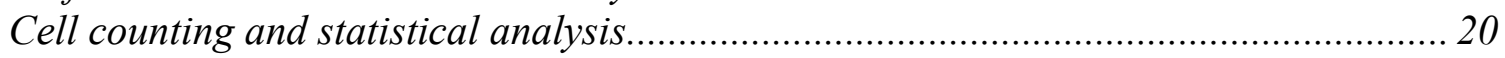

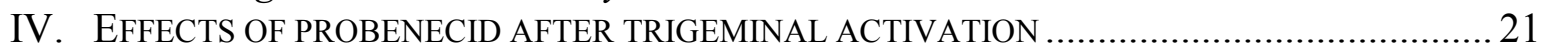

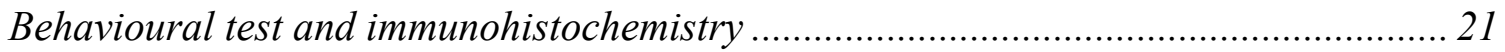

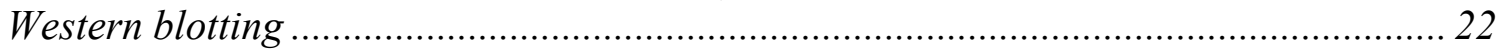

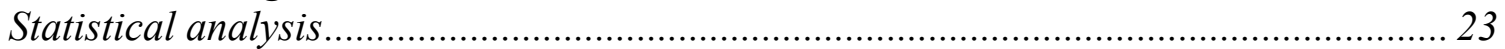

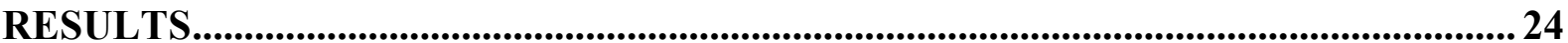

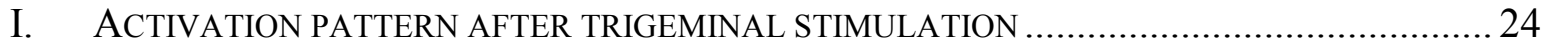

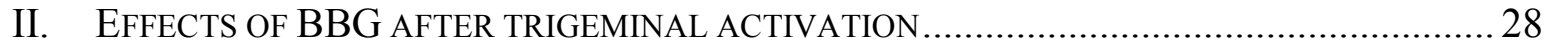

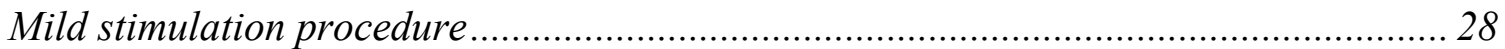

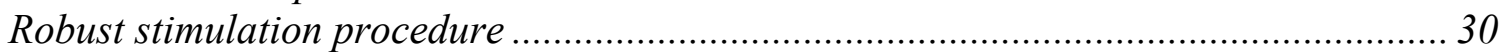

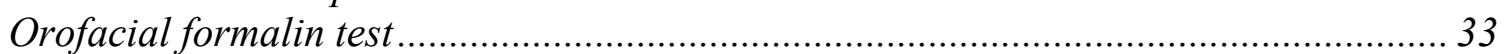

III. EFFECTS OF PROBENECID AFTER TRIGEMINAL ACTIVATION .............................................. 38

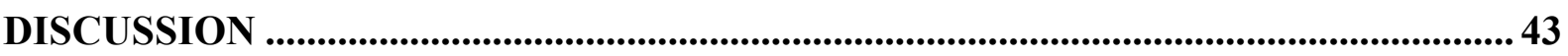

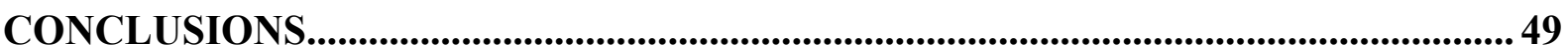

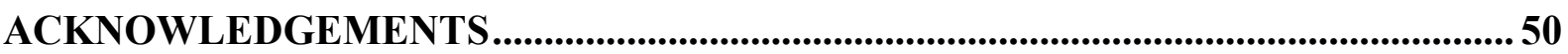

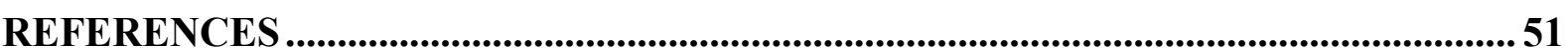




\section{List of abbreviations}

$\begin{array}{ll}\text { BBG } & \text { Brilliant Blue G-250 } \\ \text { CGRP } & \text { calcitonin gene-related } \\ & \text { peptide } \\ \text { CSD } & \text { cortical spreading depresion } \\ \text { DLPAG } & \text { dorsolateral periaqueductal } \\ & \text { grey }\end{array}$
PAG periaqueductal grey
PANX1 pannexin-1

DMPAG dorsomedial periaqueductal grey

PBS phosphate buffered saline

PBST phosphate-buffered saline containing 1\% Triton X-100

PGE $_{1}$ prostaglandin $\mathrm{E}_{1}$
PGE $_{2} \quad$ prodtaglandin $\mathrm{E}_{2}$
PROB probenecid
DR nucleus raphe dorsalis
ESTG electrical stimulation of the trigeminal ganglion

FDA U.S. Food and Drug

Administration

RVM rostral ventromedial medulla

s.c. subcutaneous

SDS sodium dodecyl sulphate

TBST Tris-buffered saline

$\begin{array}{ll}\text { GABA } & \gamma \text {-aminobutyric acid } \\ \text { i.p. } & \text { intraperitoneal } \\ \text { i.v. } & \text { intravenous }\end{array}$
IL-1及 interleukin-1 $\beta$
IR immunoreactive

LC locus coeruleus

LPAG lateral periaqueductal grey

MA migraine with aura

MO migraine without aura

MRP4 multidrug resistanceassociated protein

NGS normal goat serum

NMDA N-methyl-D-aspartate

nNOS neuronal nitric oxide synthase

NRM nucleus raphe magnus

P2X7-R P2X7 receptor containing $0.2 \%$ Tween 20

TNC nucleus tractus spinalis nervi trigemini pars caudalis/ caudal trigeminal nucleus

TRPA1 transient receptor potential channel subtype A member 1

TRPV1 transient receptor potential channel subtype V member 1

TRPV2 transient receptor potential channel subtype $\mathrm{V}$ member 2

VLPAG ventrolateral periaqueductal grey 


\section{Summary}

Migraine is a common neurological disorder with a high social burden and unknown pathomechanism. The trigeminal systems plays a crucial part in the pathological processes of the disease, therefore the knowledge about the functioning and modulation options of this system is essential for developing new therapeutic options against this condition.

One of the animal models used in migraine research is the electrical stimulation of the trigeminal ganglion (ESTG). In this model we examined the activation pattern of the brain stem nuclei, previously regarded as migraine generators in human studies.

To find new targets modulating the trigeminal nociceptive pathway we examined the effects of Brilliant Blue G-250 (BBG) and probenecid (PROB) on the number of c-Fos immunoreactive cells and on the changes in calcitonin gene-related peptide and neuronal nitric oxide synthase immunoreactivity in the caudal trigeminal nucleus. BBG is a P2X7 receptor antagonist, which receptors were previously shown to be involved in nociceptive processing. PROB is an inhibitor of numerous organic anion transporters and multidrug resistance-associated proteins, and was proposed to be antinociceptive. BBG was tested in the ESTG model in two stimulation paradigms, a mild and a robust one, and in the orofacial formalin test, while PROB was tested in the formalin test only.

After ESTG the activation pattern of the brainstem nuclei was not similar to the pattern seen during migraine attacks in human functional studies, suggesting that the activation of the trigeminal system in the rat does not lead to the activation of the migraine generator nuclei.

BBG was effective in decreasing the activation of the second order trigeminal neurones only in the robust stimulation paradigm of ESTG, which suggest that P2X7 receptors more likely play a fine-tuning role in of the trigeminal system, and are not general modulators.

PROB had a clear antinociceptive behavioural effect and was able to decrease trigeminal activation after formalin, suggesting that one or even more of its molecular targets play a crucial part in trigeminal nociceptive processing.

Our results provide important information about the functioning of the trigeminal system, thus contribute to the understanding of pathological processes underlying headache and migraine. 


\title{
Introduction
}

\author{
I. Migraine \\ General characteristics
}

Migraine is defined as a neurological disease characterised by spontaneous recurrent attacks of headache, lasting for 4-72 hours without treatment with accompanying symptoms of nausea, vomiting, photo-and phonophobia (1). In most of the cases the headache is unilateral and throbbing in nature, with moderate or severe intensity, and often worsened by physical activity (1). Migraine belongs to the family of primary headache disorders, implying that no other cause can be associated with the development of the disease. Despite the enormous research effort the exact pathomechanism of the disorder is still unknown.

As a common neurological condition, migraine affects $14.7 \%$ of the population in Europe (2), mainly young adult people between 18 and 55 years of age (3). Therefore, the economical burden caused by the disease is significant (4), and underlines the need for further research seeking the answers for open questions in its pathomechanism.

The migraine attack can be divided into four, often overlapping phases: the prodrome or premonitory phase, the aura phase, the headache phase and the postdrome. Of the four phases the aura and the headache phases are the most well studied, less is known about the pro-and postdrome phase. The aura is present in one-third of patients (5), giving the base of the main classification of the two most prevalent migraine types: migraine with and without aura (MA and MO respectively) (1).

Migraine is more prevalent in women compared to men; after puberty the migraine occurrence ratio is approximately 3:1 (5). This fact suggests the involvement of female gonadal hormones in its pathomechanism (6).

Among others, family aggregation studies suggest, that migraine has a predominantly genetic background $(7,8)$, but the exact genes were identified only for the very rare monogenic forms (familial hemiplegic migraine type I, II, and III). In case of MA and MO studies suggest that multiple loci are affected with some overlapping genes in both forms, but might also support the possibility, that the two common forms of migraine have different genetic background (9-11). 


\section{Pathomechanism}

One of the challenges of migraine research is to consider all four phases as one entity, and finding a complex explanation to all of the occurring symptoms. Several theories emerged during the years to explain the pathomechanism of migraine, and the subsequent research induced by these theories resulted in important discoveries about the occurring processes. However no theory proved to be completely applicable to explain the cause and all the detailed aspects of the disease.

The first concept was proposed in the 1950's based the work of Ray and Wolff regarding the observations of the pain sensitive structures of the head (12). The main hypotheses of this theory were, that intracranial vasospasm is causing the aura symptoms, while extracranial vasodilatation is responsible for the migraine pain (13). The theory promoted intensive research in the field of vascular changes in migraine, and indirectly facilitated the development of the triptans, the migraine specific drugs approved by the FDA. However the vascular changes do not explain all of the symptoms of the attack.

The neurogenic theory stated that the primary origin of migraine is neuronal (14). The vascular and neuronal theory was combined based on the work of Moskowitz and colleagues (15-17). In this view the primary cause of the headache derived from the activation of the primary afferent neuronal fibres, which released inflammatory substances, affecting vascular tone and leading to plasma protein extravasation, resulting in neurogenic inflammation. The initiating step, however is not completely known in these models.

With the widespread availability and development of functional imaging methods the precise mapping of the migrainous brain became possible. Weiller and colleagues detected, that during spontaneous migraine attacks the dorsolateral pons and the dorsal midbrain involving the nuclei nucleus raphe magnus (NRM), nucleus raphe dorsalis (DR) locus coeruleus (LC) and the periaqueductal grey matter (PAG) are activated and their activation persist even after the successful pain control with sumatriptan (18). These observations support the neuronal origin of the disease and were confirmed by other research groups in case of spontaneous and also during nitroglycerin induced migraine attacks (19-21). This activation was shown to be migraine specific, as was not present after injection of capsaicin to the head (22). These observations provided the foundation of the theory that the above mentioned nuclei initiate the migraine attack, they are the "migraine generators". Although, it 
is not clear whether this activation is the cause of the attack, or just a secondary phenomenon related to pain sensation.

These activated nuclei are part of the endogenous pain modulating system. The NRM is found in the ventral midline of the brainstem and gives rise to the main modulating pathway affecting directly the spinal cord dorsal horn, and the caudal trigeminal nucleus (TNC) (23). It contains serotonin and GABA positive cells, and the two neurotransmitters often co-localize there (24). Together with the paragigantocellular reticular nucleus and the gigantocellular reticular nucleus pars alpha it forms the rostral ventromedial medulla (RVM). In the RVM ON, OFF and NEUTRAL cells can be found, identified by their firing pattern related to nociceptive stimuli (25). ON cells begin to fire just before the happening of a nociceptive withdrawal reflex; while OFF cells cease firing at the start of the same reflex. The firing pattern of NEUTRAL cells remains unchanged. Based on these observations ON cells are thought to be pro- while OFF cells are thought to be antinociceptive, but the mechanisms by which these cells are able to modulate nociceptive processing are under intensive research (26).

The PAG sends a scarce input to the trigeminal complex directly (27), but it has dense projections to the NRM, and similarly the ON, OFF and NEUTRAL cells can be identified also in the PAG (28). Functionally the PAG is organized into columns, which have different neurotransmitter content and projection sites (29). A number of studies suggest that the sensation of pain and the reactions evoked by a painful stimulus are modified according to the environmental factors and to the behavioural state of the individual. The PAG is considered as one of the integrators of different sensory and behavioural information (30). Stimulation of the PAG was able to reduce the activity of trigeminal primary afferents in cats, confirming its involvement in trigeminal pain processing (31).

Locus coeruleus is one of the main noradrenergic nuclei in the brain, sends dense projections to both higher order brain areas and to the spinal cord dorsal horn (32). Stimulation of the LC can produce analgesia (33), and may exert similar effector function in pain modulation as the RVM (34). It has dense projections to the TNC (35) and to the intraand extracranial vasculature, suggesting a possible involvement in the vascular events of migraine (36). 
The DR is embedded between the ventrolateral columns of the PAG, and is the largest serotonergic nucleus in the mammalian brain (37). Similarly to the previously mentioned nuclei, stimulation of the DR can also produce analgesia in experimental animals (38). The DR can exert its modulatory effect on the nociceptive processing directly in the trigeminal system $(39,40)$, or in a more feasible way throughout its dense projections to the NRM (41). All of the "migraine generator" nuclei are important elements of the pain modulating system, however their initiating role in the migraine attack remains elusive.

An intensively investigated process in migraine pathomechanism is the cortical spreading depression (CSD). During this phenomenon a spreading depolarization wave moves across the cortex from the occipital to the frontal direction, followed by hyperpolarisation and blood flow changes. Based on the findings of Leao about the velocity of CSD waves (42) and the results of functional imaging studies it was suggested that CSD is the pathophysiological substrate of migrainous aura $(43,44)$. Whether the CSD is capable of initiating a migraine attack or not, is in the focus of current migraine research, but data gathered so far are controversial.

From the data detailed above we can conclude that the pathophysiology of migraine is still an interesting and highly controversial research area. To understand the mechanism of headache generation during the attack, the knowledge about the functioning of the trigeminal system, which conveys sensory information from the head, is essential.

\section{Animal models of trigeminal activation}

Based on experimental data the most debilitating aspect of the migraine attack, the headache is due to the activation of the primary afferents of the trigeminal system (45). The primary afferents of the fifth/trigeminal nerve innervate the oral, facial and cranial territories of the head and convey nociceptive information to the central nervous system. The trigeminal nerve is divided into three branches, the ophthalmic (V1), the maxillary (V2) and to the mandibular (V3) branch. Nociceptive information from the branches is transmitted throughout the sensory ganglion of the trigeminal system, the trigeminal or Gasserian ganglion, which is situated at the anterior surface of the pars petrosa of the temporal bone. The central 
projections of the primary nociceptive neurons terminate in the superficial layers of the upper cervical segments and in the TNC, forming the trigemino-cervical complex.

The brain parenchyma itself is not reactive to painful stimuli, the vasculature and the meninges are the origin of nociceptive signals intracranially (12). The supratentorial dural and pial tissues are mainly innervated by the ophthalmic branch of the trigeminal nerve while the infratentorial parts are innervated by the upper cervical nerves $(C 2, C 3)(46)$.

To study the functioning of the trigeminal system and to reveal the mechanism involved in the generation of migrainous headache animal models are used. Unfortunately, no animal model has been developed so far which would be able to completely reflect all the genuine characteristic of a human migraine attack, however they are very useful in the testing of distinct features of headache mechanisms and the activation of the trigeminal system.

One of the animal models of trigeminal activation is the electrical stimulation of the trigeminal ganglion (ESTG). In 1987 Markowitz and colleagues showed, that neurogenic plasma protein extravasation can be evoked by ESTG (15), and suggested that the release of neuropeptides is a key factor in this phenomenon. This was confirmed by detecting increased calcitonine gene-related peptide (CGRP) levels from the superior sagittal sinus in rats (47) and from the external jugular vein from cats and human patients after ESTG (48). Sumatriptan and ergot alkaloids, which are also used in migraine therapy, were able to inhibit plasma protein extravasation $(49,50)$ suggesting that neurogenic inflammatory mechanisms are participant is the process of migrainous headache generation. The activation of the second order neurones at the level of the TNC was also shown after ESTG with the aid of the detection of the messenger RNA of the immediate early gene $c$-fos (51) and also with the detection of the protein product c-Fos (52). C-Fos is activated shortly after different stimuli and is widely used to detect nociception related activity changes in the trigeminal system (53). In the ESTG model the amount of c-Fos immunoreactive cells in the TNC is highly dependent on the applied stimulation intensity and frequency (54) indicating that the utilization of various stimulation settings may be advisable.

Subcutaneous (s.c.) injection of formalin is commonly used to examine inflammatory pain reactions in animals both at the behavioural and at the molecular level (55). A specific version of the formalin test is when the stimulus is applied to the whisker pad of the animals leading to the activation of the trigeminal system, the orofacial formalin test (56). The 
behavioural reaction to the injected formalin is the immediate shaking of the head and scratching the injected area with the ipsilateral fore or hind paw. The behavioural response presents in two phases. The first phase is short-lasting and begins immediately after the application of formalin, and thought to be caused by the direct effect of formalin on nociceptors, mainly on $\mathrm{C}$ fibers (57). The second phase begins after a quiescent period, is more prolonged and presumably caused by inflammatory mechanisms (58). Besides the behavioural effects, a clear activation of the trigeminal system is also detectable in the orofacial formalin test. The c-Fos increase demonstrated activation presents according to the somatotopy at the level of the TNC (59). The test is used to identify possible new migraine therapeutic targets $(60)$.

\section{Purinergic receptors and nociceptive processing}

The purine molecule ATP was proposed to have an important role in the regulation of nociceptive transmission (61). Purinergic receptors sensitive for ATP are divided into two main classes, the P2X and P2Y receptors and their agonist and antagonists have been proven to be effective in modulating different pain conditions, e.g. neuropathic and inflammatory pain (62). Among the ligand-gated $\mathrm{P} 2 \mathrm{X}$ receptors the $\mathrm{P} 2 \mathrm{X} 7$ receptor (P2X7-R) has been intensively studied in different pain states. The P2X7-R is a non-selective cation channel, unique among the P2X-Rs by virtue of its long $\mathrm{C}$ terminal domain, and its ability to open a pore permeable to molecules up to $900 \mathrm{Da}$ (63). Experiments with knock-out mice have revealed that absence of the P2X7-R leads to the disappearance of mechanical and thermal hypersensitivity in models of neuropathic and inflammatory pain, whereas normal nociceptive processing is retained (64). P2X7-R antagonists have been examined in similar models, and the results underline the importance of the P2X7-R in chronic pain conditions $(65,66)$. Results from an acute inflammatory pain model suggest that the P2X7-R may participates in the development of central sensitization (67), a common feature in the trigeminal system during migraine attacks manifested by the presence of allodynia (68).

However, little information is available as concerns the role of P2X7-Rs in the trigeminal system. In a model of orofacial pain, the chronic constriction injury model, inhibition of the P2X7-R led to a decrease in tactile allodynia through a p38 mitogen-activated protein kinase-dependent mechanism (69). Gölöncsér and Sperlágh 
recently reported that blockade of P2X7-Rs in mice by Brilliant Blue G-250 (BBG) reduced thermal hyperalgesia after systemic nitroglycerin administration (70). BBG is a selective, non-competitive P2X7-R antagonist, with good blood-brain barrier permeability (71) and was earlier proposed to be effective in neuropathic pain models (72). These results lend support to the theory that P2X7-Rs may play a crucial part in the development of headache disorders.

\section{Probenecid and nociceptive processing}

Probenecid (PROB, $p$-(di-n-propylsulfamyl)benzoic acid) has primarily been used to enhance penicillin concentration in the serum by inhibiting the renal clearance throughout the inhibition of organic anion transporters (73) and to increase the renal excretion of uric acid to treat gout (74). Data also suggest that PROB may has anti-inflammatory and antinociceptive properties, related to several mediators participating in nociceptive and inflammatory conditions. One of this mediators is the multidrug resistance-associated protein 4 (MRP4), an organic anion transporter of which PROB is an inhibitor (75). MRP4 plays a crucial role in the release of prostaglandin $\mathrm{E}_{1}\left(\mathrm{PGE}_{1}\right)$ and prostaglandin $\mathrm{E}_{2}\left(\mathrm{PGE}_{2}\right)$ key inflammatory mediators (76), thus PROB could modulate prostaglandin related inflammatory processes. Furthermore, PROB is an agonist of the transient receptor potential channel subtype A member 1 (TRPA1) (77) and the transient receptor potential channel subtype V member 2 (TRPV2) (78), both being involved in modulation of pain sensation $(79,80)$. The agonistmediated desensitization of these TRPA1 and TRPV2 channels can occur $(77,81)$, resulting in analgesia. In contrast, it was also proposed that probenecid can elicit the pain induced by inflammation through its agonism on TRPV2 (78). PROB also blocks the pannexin-1 channel (PANX1) (82), which is involved in neuronal inflammatory processes (83). Several studies suggest that PANX1 and the P2X7-R are in interaction during inflammatory processes $(84$, 85), and their modulation could provide new therapeutic options in the treatment of chronic pain states (86). These results strongly suggest that PROB might has a mitigating effect on inflammatory and nociceptive mechanisms. 


\section{Aims}

Our aims in the present study were

I. To evaluate the activation pattern of the migraine generator nuclei after electrical stimulation of the trigeminal ganglion at different survival times in the rat.

II. To examine the effects of a purinergic P2X7 receptor antagonist, BBG on the activation in the TNC caused by two different electrical stimulation settings.

III. To test the effects of BBG in the orofacial formalin test of the rat both at the behavioural and on the molecular level.

IV. To detect the effects of probenecid on behaviour and on nociceptive activation in the orofacial formalin test of the rat. 


\section{Materials and methods}

\section{Animals}

The procedures used in all experiments followed the guidelines of the eighth edition of the Guide for the Care and Use of Laboratory Animals and the Use of Animals in Research of the International Association for the Study of Pain and the directive of the European Economic Community (86/609/ECC). Experiments were approved by the Committee of Animal Research at the University of Szeged (I-74-14-16/2008; I-74-12/2012) and the Scientific Ethics Committee for Animal Research of the Protection of Animals Advisory Board (XI./15.1/02384/001/2007; XXIV/352/2012). Adult male Sprague-Dawley rats were used. They were raised and maintained under standard laboratory conditions on a $12 \mathrm{~h}$ lightdark cycle, with tap water and rat chow available ad libitum. Suffering of the animals and the number of animals used were kept at minimum.

\section{Activation pattern after trigeminal stimulation}

\section{Experiments}

Twenty-six rats were divided into two groups. The animals in the first group $(n=13)$ were deeply anaesthetized with chloral hydrate $(400 \mathrm{mg} / \mathrm{kg})$ and placed in a stereotaxic frame (Stoelting Co.). A hole was drilled with a dental drill 3.2-3.4 mm posterior to and 2.8-3.2 mm laterally from the bregma. A concentric bipolar electrode (FHC Inc., CBBRE75) was lowered to the right trigeminal ganglion, and maintained in it for $30 \mathrm{~min}$. After surgery, the animals were kept under deep anaesthesia, additional doses of chloral hydrate if needed being given. They were returned back to their cages and were kept warm.

The animals in the second group $(n=13)$ underwent a similar surgical procedure, but were additionally stimulated for $30 \mathrm{~min}$ with square twin pulses at $10 \mathrm{~Hz}, 0.5 \mathrm{~mA}$, with a pulse duration of $5 \mathrm{~ms}$ (Medicor, Electrostimulator ST3). The applied stimulation frequency induces depolarization, thus rapid firing of the pseudounipolar neurones of the trigeminal ganglion, and the intensity applied ensures that the area of the stimulation covers the entire trigeminal ganglion (87). 
The correct placement of the electrode was checked by the twitching of the jaw when the electrode reached the ganglion, and was confirmed during the autopsy. The same was done in the subsequent stimulation paradigms too.

From both groups 7 animals were perfused transcardially two hours after the placement of the electrode, while 6 animals were perfused four hours after the electrode placement, with $100 \mathrm{~mL}$ cold $0.1 \mathrm{M}$ phosphate buffered saline (PBS), followed by $500 \mathrm{~mL}$ $4 \%$ paraformaldehyde in $0.1 \mathrm{M}$ phosphate buffer. The brains and cervical spinal cord were removed and postfixed overnight in the same fixative.

\section{Immunohistochemistry}

After cryoprotection $30 \mu \mathrm{m}$ thick serial sections were cut $1 \mathrm{~mm}$ rostrally and $5 \mathrm{~mm}$ caudally from the obex, representing the TNC. The series were collected into 18 wells, the distance between consecutive sections was $540 \mu \mathrm{m}$.

Similarly $30 \mu \mathrm{m}$ thick serial sections were cut from the brainstem, the series started $1.5 \mathrm{~mm}$ rostrally from the obex and 30 series were cut from each animal. The series were collected into 10 wells, the distance between consecutive sections therefore being $300 \mu \mathrm{m}$.

The free-floating sections were rinsed in PBS and immersed in methanol containing $0.3 \% \mathrm{H}_{2} \mathrm{O}_{2}$ for $30 \mathrm{~min}$. After several washes in PBS containing 1\% Triton X-100 (PBS-T), the sections were blocked for 1 hour in PBS-T containing 10\% normal goat serum (NGS). Sections from the TNC were incubated for one night at room temperature in PBS-T containing 2\% NGS and 1:2000 anti c-Fos primary antibody (rabbit anti-human c-Fos polyclonal antibody, Santa Cruz Biotechnology, sc-52), while sections from the brainstem were incubated for 2 nights at $4^{\circ} \mathrm{C}$ in PBS-T containing 2\% NGS and 1:1000 anti c-Fos primary antibody (same as previously). The immunohistochemical reaction was visualized by the avidin-biotin kit of Vectastain (Vector Laboratories Inc., PK-6101) and stained with nickel ammonium sulphate-intensified 3,3'-diaminobenzidine for sections of the TNC and 3,3'-diaminobenzidine without intensification for sections of the brainstem. The specificity of the immune reaction was checked by omitting the primary antiserum. On brainstem sections toluidine blue counterstaining was applied to facilitate cell counting. 


\section{Cell counting}

The c-Fos-immunopositive cells were counted according to Paxinos and Watson (88) in the TNC, NRM, DR, ventrolateral periaqueductal grey (VLPAG), lateral periaqueductal gray (LPAG), dorsolateral periaqueductal gray (DLPAG), dorsomedial periaqueductal gray (DMPAG) and LC separately. Sections were examined under a Zeiss Axio Imager M2 Upright Microscope (Carl Zeiss MicroImaging,) supplied with an AxioCam MRc camera (Carl Zeiss MicroImaging). Photographs were taken on 20X magnification, using the MosaiX program feature of the AxioVision software. Cells were counted and area of the different nuclei were delineated and measured on one series of sections from the TNC and from the brainstem in each animal. Cell counting was made by an investigator blinded to the stimulation procedure, furthermore stimulated and unstimulated sides of the sections were counted separately.

\section{Statistical analysis}

For the different nuclei, cell counts per side and per series were summed and divided by the corresponding area. In this way the cell count per $\mu \mathrm{m}^{2}$ was calculated for each side and for each series in each animal.

In case of the TNC, counts were averaged by animal and statistical comparison was made using ANOVA (SPSS Statistics 17.0 for Windows, Games-Howell post hoc test).

In case of other paired nuclei of the brainstem the first step was to determine if there is any difference between the sham and stimulated sides using Student's t-test. There was no significant difference in any of the nuclei, therefore, data from separate sides were pooled. These data were than compared using ANOVA (SPSS Statistics 17.0 for Windows, Scheffe post hoc test).

Statistical comparison in the NRM and DR was also conducted by ANOVA (SPSS Statistics 17.0 for Windows, Scheffe post hoc test).

Linear regression analysis was conducted to examine if there is any correlation between the cell number changes in the TNC and NRM (SPSS Statistics 17.0 for Windows). 


\section{Effects of BBG after trigeminal activation}

\section{Mild stimulation procedure}

Twenty-four animals (250-300 g) were used. Half of the animals received an intravenous (i.v.) injection of $50 \mathrm{mg} / \mathrm{kg} \mathrm{BBG}$, while the other half was injected with the vehicle of BBG, physiological saline. Two hours after the BBG or saline injection, the animals were deeply anesthetized with chloral hydrate $(400 \mathrm{mg} / \mathrm{kg})$ and the surgical procedure as stated in the previous section was performed. Half of the animals from the saline-treated group (5SStim) and half of the animals from the BBG group (5BStim) were electrically stimulated for $5 \mathrm{~min}$ with $5 \mathrm{~Hz}, 0.5 \mathrm{~mA}, 0.5 \mathrm{~ms}$ delay twin pulses. The other animals from both groups were used as sham animals: the electrode was lowered to the right trigeminal ganglion for $5 \mathrm{~min}$, but no stimulation was performed (5SSham and 5BSham groups). After both procedures, the animals were returned to their home cages and maintained under deep anaesthesia covered by a warming blanket for 2 hours.

\section{Robust stimulation procedure}

Twenty-one animals (250-300 g) were used; the treatment and surgical procedures were identical to the previous ones, except that the stimulation parameters of $10 \mathrm{~Hz}, 0.5 \mathrm{~mA}$, $0.5 \mathrm{~ms}$ delay twin pulses were applied for $30 \mathrm{~min}$, and the animals were maintained under deep anaesthesia, covered by a warming blanket, in their home cages for 4 hours from the beginning of the stimulation. These parameters are equivalent to the ones applied in the activation pattern experiments.

Overview of the robust stimulation groups:

Saline + 30-min sham: 30SSham $(\mathrm{n}=6)$

Saline +30 -min stimulation: 30SStim $(n=5)$

BBG + 30-min sham: 30BSham $(n=4)$

BBG + 30-min stimulation: 30BStim $(n=6)$

\section{Orofacial formalin test}

Rats ( $\mathrm{n}=52,200-240$ g) were injected i.v. either with $50 \mathrm{mg} / \mathrm{kg}$ BBG or with physiological saline. One hour and fifty minutes later, the animals were placed in a $30 \times 30 \times 30$ 
cm box, with mirrored walls for the monitoring of behavioural activity. After $10 \mathrm{~min}$ of habituation, the animals were taken out of the box and under minimal restraint were injected s.c. with $50 \mu \mathrm{L}$ of either physiological saline (SSal and BSal groups) or with $1.5 \%$ formalin (SForm and BForm groups) to the right whisker pad. After the injection, they were returned immediately to the box and their behaviour was monitored for 45 min under video surveillance. The injection of formalin causes a behavioural response, which consists of rubbing and scratching the injected whisker pad with the ipsilateral fore- or hindpaw. The rate of this behaviour correlates with the pain sensation caused by formalin (56). The 45-min period was divided into $15 \times 3-$ min blocks, and the total time spent rubbing the injected whisker pad, measured in seconds was taken as the nociceptive score in the given block. The normal grooming activity of the saline-treated animals was measured as control. After the monitoring period, the animals were returned into their home cages and maintained under standard laboratory conditions until perfusion, which was performed under deep chloral hydrate anaesthesia 4 hours after the whisker pad injections.

\section{Perfusion and immunohistochemistry}

The animals were perfused and sections from the TNC were prepared as previously described. The same staining method was applied as detailed earlier for c-Fos. For the CGRP staining the procedure was identical except for the primary antibody, which was used in a dilution of 1:20000 (rabbit anti-rat CGRP polyclonal antibody, Sigma-Aldrich C8198) overnight at room temperature.

\section{Cell counting and statistical analysis}

Cells immunopositive for c-Fos in the laminae I-II of the entire TNC were counted on a Nikon Optiphot-2 light microscope under a 20x objective.

CGRP-stained sections were photographed with a Zeiss AxioCam MRc Rev.3 digital camera attached to a Zeiss AxioImager M2 microscope. Digital images were taken with a 20X objective in TNC laminae I-II, and the area covered by CGRP-immunoreactive fibres was measured through the use of ImageProPlus 6.2 software (Media Cybernetics Inc.) by an observer blinded to the treatment procedures. 
The cell count results were aligned according to the rostro-caudal location of the section based on the anatomical observations compared to the atlas of Paxinos and Watson (88). Data from different levels of the TNC were handled separately, and analysed by twoway repeated measures ANOVA. The group were used as the between-subject factor and the levels (-13.89, -14.43, -14.97, -15.51, -16.05, -16.59, -17.13, -17.67, -18.21 mm from bregma) as the within-subject factor for the analysis.

When Mauchly's test of sphericity proved to be significant, the Greenhouse-Geisser correction was performed. Pairwise comparisons of group means were performed on the basis of estimated marginal means with Sidak adjustment for multiple comparisons.

The sums of the areas covered by CGRP-immunoreactive fibres were compared between groups according to the different levels, two-way repeated measures ANOVA being used as detailed above.

Nociceptive scores from the behavioural study were compared block by block through two way repeated measures ANOVA. Groups were used as between-subject factor and blocks (1-15) as within-subject factor for the analysis. Other statistical parameters were identical to those mentioned above.

For the comparison of the nociceptive scores in the two phases of the formalin test one-way ANOVA was used followed by the Tamhane post hoc test. The first block of three minutes was considered as the first phase, while the sum of the blocks 5-11 was the second phase.

Statistical analyses were carried out with IBM SPSS Statistics, version 20 (IBM Corporation) software. All tests were two-sided, and $\mathrm{p}<0.05$ was considered to be statistically significant.

\section{Effects of probenecid after trigeminal activation}

Behavioural test and immunohistochemistry

Sixty rats weighing 200-250 g were used, they were divided into two groups $(n=30$ per group). The animals in the Placebo group received only intraperitoneal (i.p.) vehicle solution (physiological saline, $1.5 \mathrm{~mL}$ ) as pre-treatment. In the PROB group, the rats were pre-treated with an i.p. injection of PROB (Sigma-Aldrich; $1 \mathrm{mmol} / \mathrm{kg}$ body weight, diluted to $1.5 \mathrm{~mL}$, $\mathrm{pH}$ 7.4). One hour after the PROB or vehicle pre-treatment, half of the animals in both groups received a s.c. injection of $50 \mu 11.5 \%$ formalin solution into the right whisker pad (Placebo- 
Form and PROB-Form), while the other half of the rats were injected with s.c. $50 \mu \mathrm{L}$ physiological saline without formalin (Placebo-Phys and PROB-Phys).

The behavioural experiments were conducted as described previously at the BBG experiments, the habituation period starting fifty minutes after the pre-treatments.

Four hours after the formalin or saline injections animals $(n=10)$ processed for immunohistochemistry were transcardially perfused as described above. After cryoprotection the TNC was sectioned into ten wells containing cold PBS, and fifteen series of sections were collected into each well. The free floating sections were processed for c-Fos immunostaining as previously described. For nNOS the applied staining procedure was identical except for the primary antibody, which was used for two nights at $4{ }^{\circ} \mathrm{C}$ in a dilution of 1:5000 (rabbit anti-rat nNOS polyclonal antibody EuroProxima, B220-1).

\section{Western blotting}

Four hours after the formalin or physiological saline injection, the rats ( $\mathrm{n}=5$ per group) were deeply anaesthetized with chloral hydrate, perfused transcardially with $100 \mathrm{ml}$ ice cold PBS and the ipsi- and contralateral side of the TNC between 0 and $-4 \mathrm{~mm}$ from the obex was removed. The samples were stored at $-80^{\circ} \mathrm{C}$. The TNC segments were sonicated in ice cold lysis buffer containing $50 \mathrm{mM}$ Tris- $\mathrm{HCl}, 150 \mathrm{mM} \mathrm{NaCl}, 0.1 \%$ igepal, $0.1 \%$ cholic acid, 2 $\mu \mathrm{g} / \mathrm{mL}$ leupeptin, $2 \mathrm{mM}$ phenylmethylsulphonyl fluoride, $1 \mu \mathrm{g} / \mathrm{ml}$ pepstatin, $2 \mathrm{mM}$ EDTA and $0.1 \%$ sodium dodecyl sulphate (SDS) (all chemicals were from Sigma-Aldrich). The lysates were centrifuged at $12,000 \mathrm{RPM}$ for $10 \mathrm{~min}$ at $4^{\circ} \mathrm{C}$ and supernatants were aliquoted and stored at $-20^{\circ} \mathrm{C}$. Protein concentration was measured with the BCA Protein Assay Kit (Novagen) using bovine serum albumin as a standard. Samples were cooled on ice during the whole procedure. Prior to loading, each sample was mixed with sample buffer, and denatured by boiling for $3 \mathrm{~min}$. Equal amounts of protein samples (20 $\mu \mathrm{g} / \mathrm{lane})$ were separated by standard SDS polyacrylamide gel electrophoresis on $12 \%$ Tris-Glycine gel and electrotransferred onto Amersham Hybond-ECL nitrocellulose membrane $(0.45 \mu \mathrm{m}$ pore size, GE Healthcare). The Page Ruler Prestained Protein Ladder (Fermentas, 10-170 kDa) was used to determine approximate molecular weights. Following the transfer, membranes were blocked for one hour at room temperature in Tris-buffered saline containing Tween 20 (TBST, MP Biomedicals) and 5\% non fat dry milk powder and then incubated in TBST 
containing $1 \%$ non fat dry milk and rabbit anti-rat interleukin-1beta (IL-1 $\beta ; \mathrm{H}-153$ ) polyclonal antibody overnight at $4^{\circ} \mathrm{C}$ or in mouse anti-rat $\beta$-actin monoclonal antibody (Calbiochem, CP01, dilution: 1:100,000, incubation: overnight at $4^{\circ} \mathrm{C}$ ). Next day after several rinses in TBST, membranes were incubated in TBST containing $1 \%$ non fat dry milk and horseradish peroxidase-conjugated anti-rabbit or anti-mouse secondary antibody (Santa Cruz Biotechnology; sc-2030 and sc-2031) for 2 hours at room temperature. Protein bands were visualized after incubation of membranes with the SuperSignal West Pico Chemiluminescent Substrate (Pierce) using Carestream Kodak BioMax Light film (Kodak).

For densitometric analyses, films were scanned and quantified using Java ImageJ $1.47 \mathrm{v}$ analysis software (National Institutes of Health). The $\beta$-actin was used as sample loading control and normalization protein as well.

\section{Statistical analysis}

Statistical analysis of the behavioural data was conducted as described at the BBG orofacial test.

The numbers of IR neurones in the various groups were compared at the 15 levels along the rostrocaudal axis by using two-way repeated measures ANOVA described in details at the BBG experiments. Since there was no significant difference in the number of c-Fosand nNOS-IR neurones between the contralateral sides in the groups injected with formalin and the contra- and ipsilateral sides in the groups injected with physiological saline (data not shown), in the course of the statistical analysis the data obtained from the contralateral sides of the groups injected with formalin were used as controls. The contra- and ipsilateral sides of the formalin-injected groups were used as the between-subject factor, and the 15 levels along the rostrocaudal axis as the within-subject factor for the analysis.

Statistical analysis of the Western blot data was carried out using one-way ANOVA followed by the Sidak post hoc test. Since there was no significant difference in band densities between the contralateral sides in the groups injected with formalin and the contraand ipsilateral sides in the groups injected with physiological saline (data not shown), in the course of the statistical analysis the data obtained from the contralateral sides of the subgroups injected with formalin were used as controls. 


\section{Results}

\section{Activation pattern after trigeminal stimulation}

Activation of the TNC after trigeminal ganglion stimulation was previously demonstrated by several studies $(54,89)$. Our results also show the clear activation of the ipsilateral side of the TNC, while on the contralateral side, and in the sham animals no significant increase in c-Fos cell number could be detected (Fig 1). The different survival times did not show significant alterations in c-Fos positive cells, however at 4 hours a decreasing tendency in the cell numbers can be observed (Fig 2).

Among the brainstem migraine generator nuclei we found significant increase in the number of c-Fos positive cells only in the NRM (Fig 3), the other nuclei (LC, DR, VL PAG, L PAG, DL PAG, DM PAG did not show alterations in c-Fos expression after stimulation (Fig 4). Furthermore, no side difference could be noticed in these nuclei, the cell numbers on the stimulated and on the control sides were similar. The different survival times did not influence the cell numbers in the different nuclei, with one exception, in the LC the c-Fos positivity decreased after 4 hours survival compared to the 2 hours survival group (Fig 4 ). The linear regression analysis did not reveal a significant connection between the increased cell number is the TNC and NRM $\left(\mathrm{R}^{2}=0.252 ; \mathrm{F}=3.708 ; \mathrm{p}=0.80\right)$. 

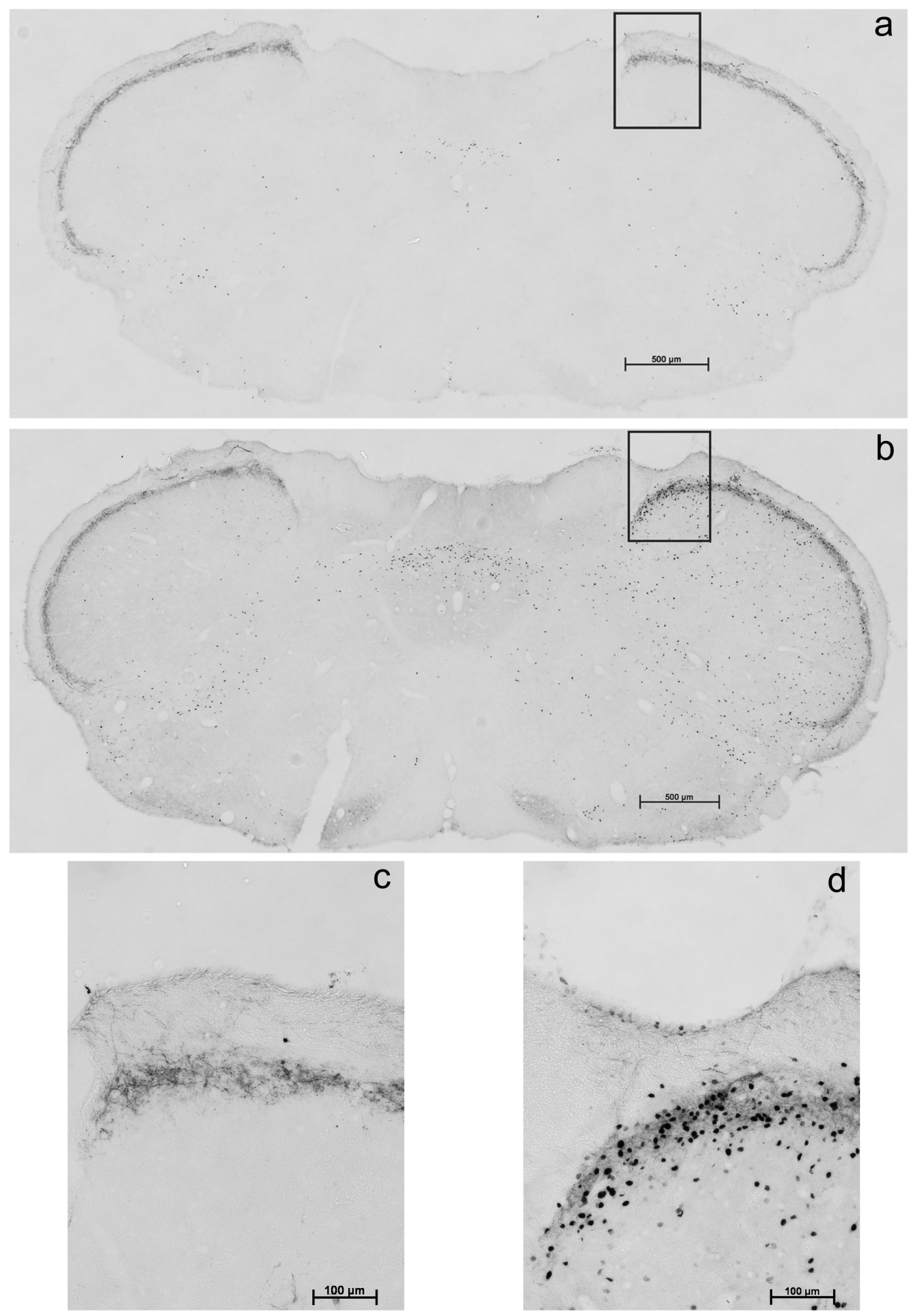

Figure 1.

Representative photos of the TNC from a sham (a, c) and from a stimulated animal (b, d) taken approximately $0.5 \mathrm{~mm}$ caudal from the obex after 2 hours survival. More cells can be detected on the electrode side of the stimulated animal (d) compared to the electrode side of the sham animal (c). Scale bars $500 \mu \mathrm{m}(\mathrm{a}, \mathrm{b}) 100 \mu \mathrm{m}(\mathrm{c}, \mathrm{d})$. 


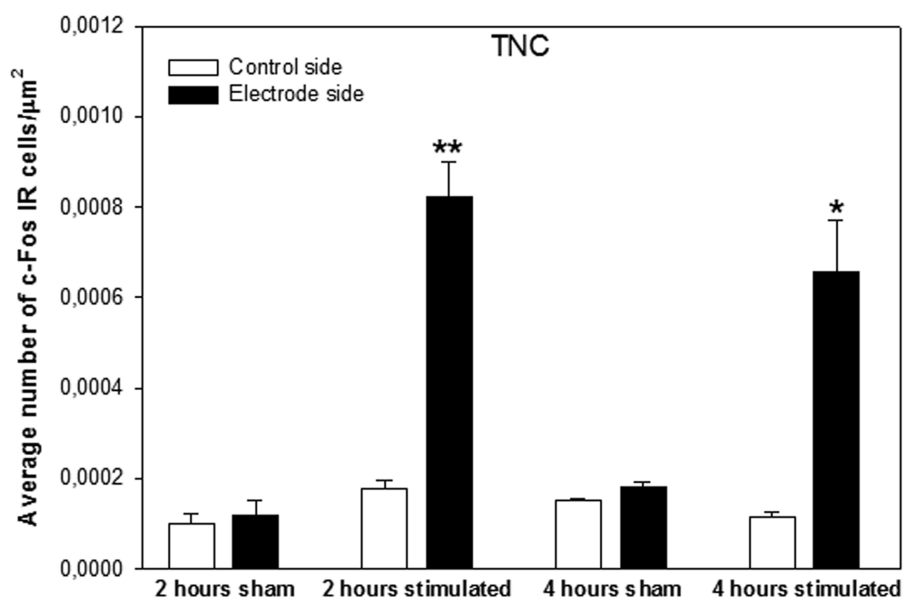

Figure 2.

Diagram showing the average number of c-Fos IR cells per $\mu \mathrm{m}^{2}$ in the TNC after 2 and 4 hours of survival after stimulation or sham surgery (group means per side + SEM). In the sham animals no increase in c-Fos cell numbers can be detected either in the electrode or in the control sides. However in both the 2 and 4 hours survival group a marked increase is present compared to the corresponding electrode sides of the sham animals. No significant difference is present between the cell numbers of the two different survival times $(* \mathrm{p}<0.05 ; * * \mathrm{p}<$ $0.01)$.

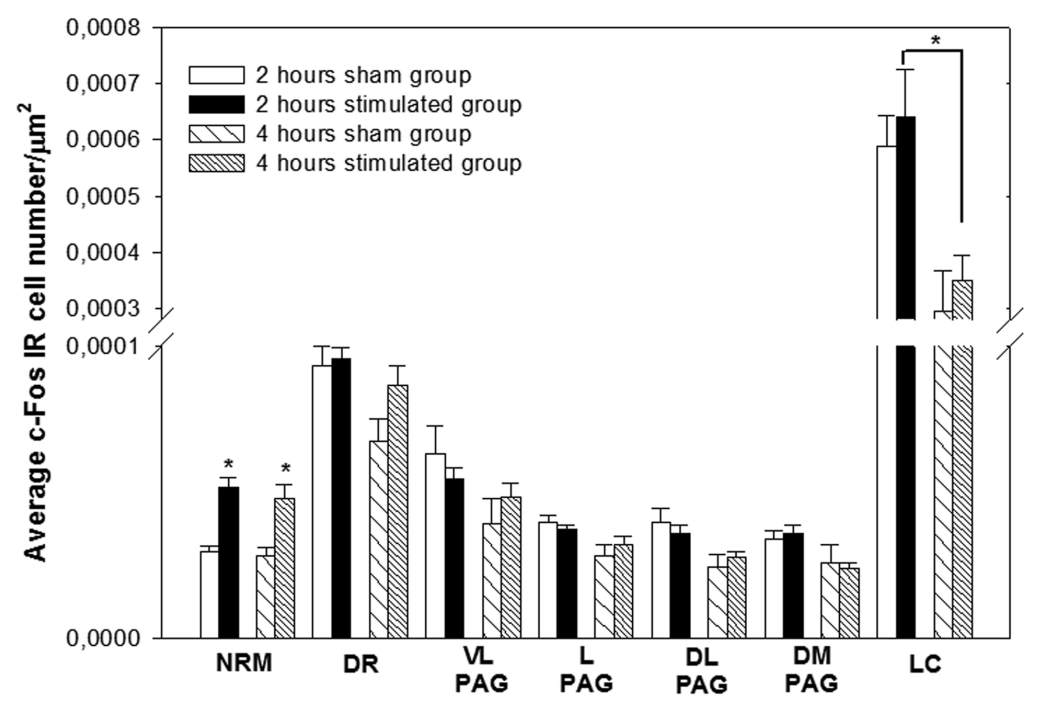

Figure 3.

Diagrams showing the average number of c-Fos IR cells per $\mu \mathrm{m}^{2}$ in brainstem nuclei (group means + SEM). The highest cell number per area ratio was present in the LC, which is not surprising, considering the small extent of this nucleus. Statistically significant alteration between the sham and stimulated group could only be detected in the NRM $\left({ }^{*} p<0.05\right)$, where the stimulation increased the number of positive cells. There is a significant difference 
$\left({ }^{*} \mathrm{p}<0.05\right)$ between the two stimulated groups in the LC, but no such difference could be observed in the other nuclei.
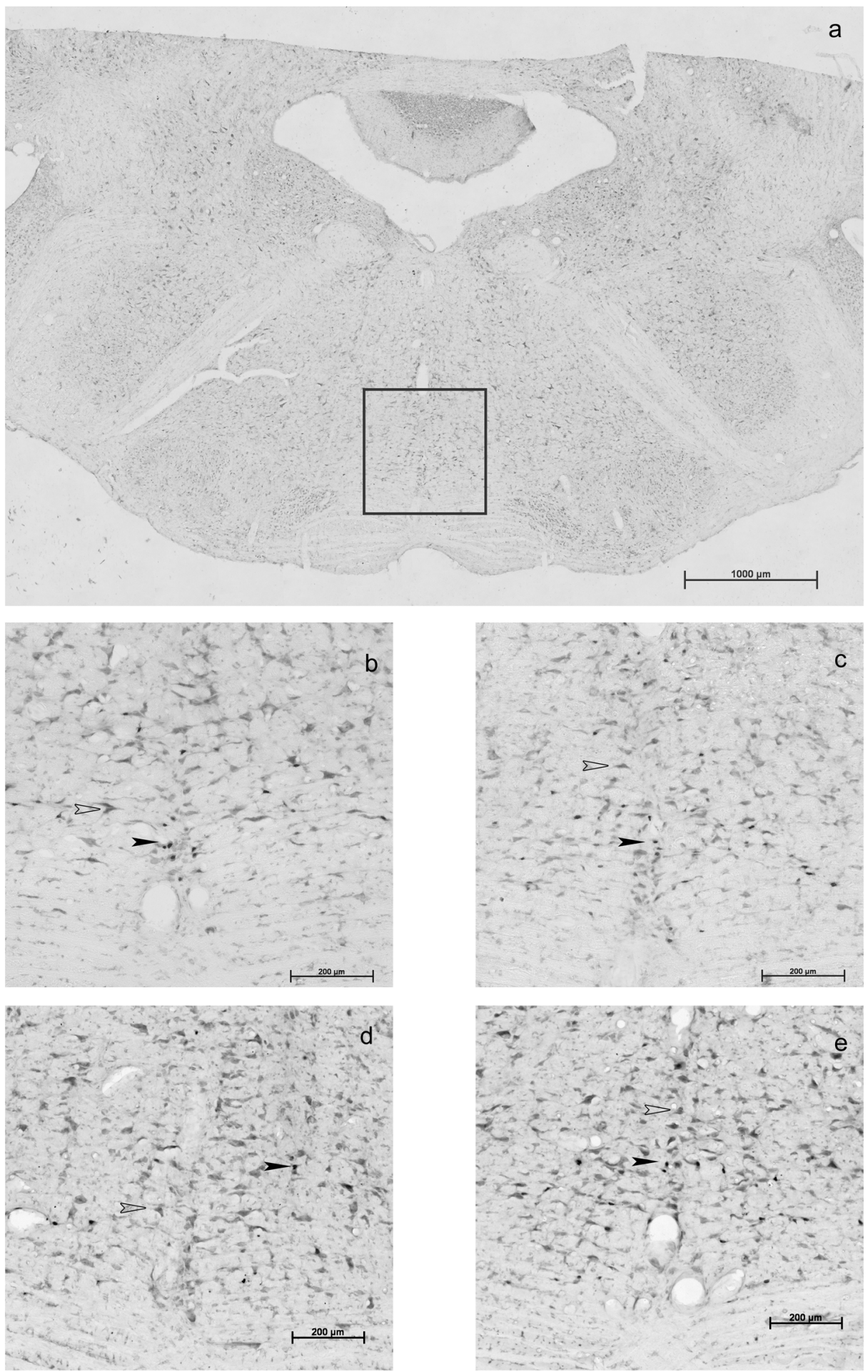

Figure 4. 
Representative photos from the NRM taken from sham (b, d) and stimulated (a, c, e) animals after 2 hours (a, b, c) or 4 hours (d, e) survival approximately $4 \mathrm{~mm}$ rostral from the obex. Filled arrowheads indicate c-Fos positive cells, while unfilled arrowheads indicate c-Fos negative cells, counterstained with toluidine blue. After 2 hours of survival, c-Fos immunoreactive cells are more numerous in the stimulated group (b, c). Four hours after the stimulation the activation of the nucleus raphe magnus is still present (e). Scale bars $1000 \mu \mathrm{m}$ (a) and $200 \mu \mathrm{m}(\mathrm{b}, \mathrm{c}, \mathrm{d}, \mathrm{e})$.

\section{Effects of BBG after trigeminal activation}

\section{Mild stimulation procedure}

As a significant interaction was found between the two investigated factors (levels and groups, $\mathrm{p}<0.01$ ) for the number of $\mathrm{c}$-Fos-immunoreactive cells in the mild stimulation paradigm, both effects could not be reported independently, whereas the group differences could be examined separately across different levels on the basis of the estimated marginal means for multiple comparisons.

The comparisons of the cell numbers from the control (left) sides for each of the four treatment groups did not reveal any significant changes (data not shown), therefore only the data for the stimulated (right) sides of the groups are presented in Fig 5 a-d. ESTG caused a significant increase in the number of c-Fos-immunoreactive cells along the whole extent of the examined region of the TNC (Fig 5 a, b, e). BBG exhibited a significant effect compared with the saline treated stimulated animals only at the level of $-13.89 \mathrm{~mm}$ from bregma (Fig 5 b, c, e).

A significant interaction was found between the groups and levels, when the area values from the CGRP measurements were examined. However, the comparisons of the groups and stimulated-control sides at different levels did not reveal any significant alteration (Fig 6). 
a

C

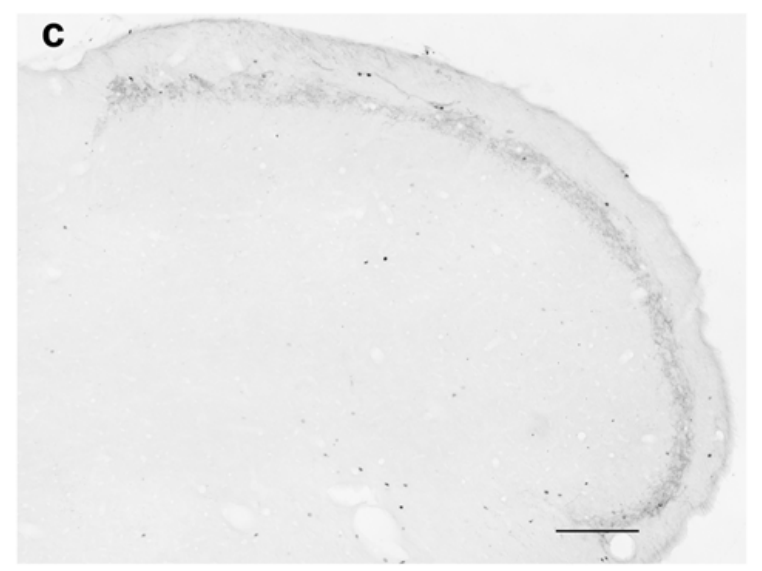

b

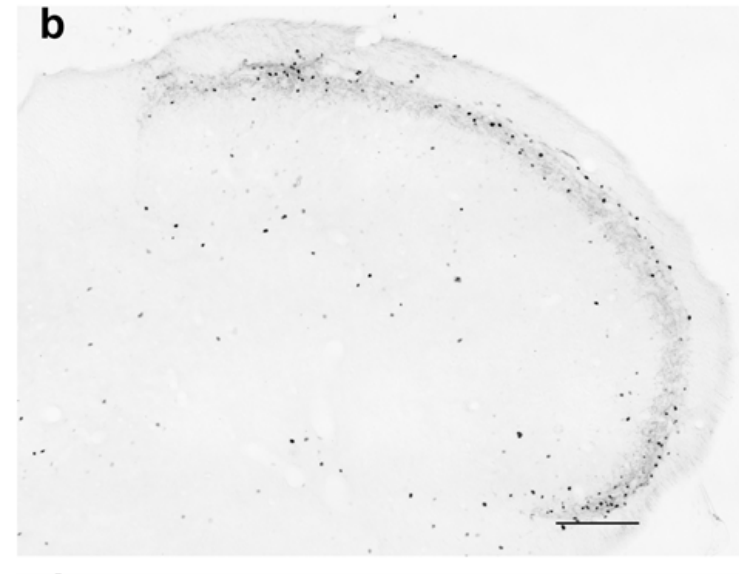

d

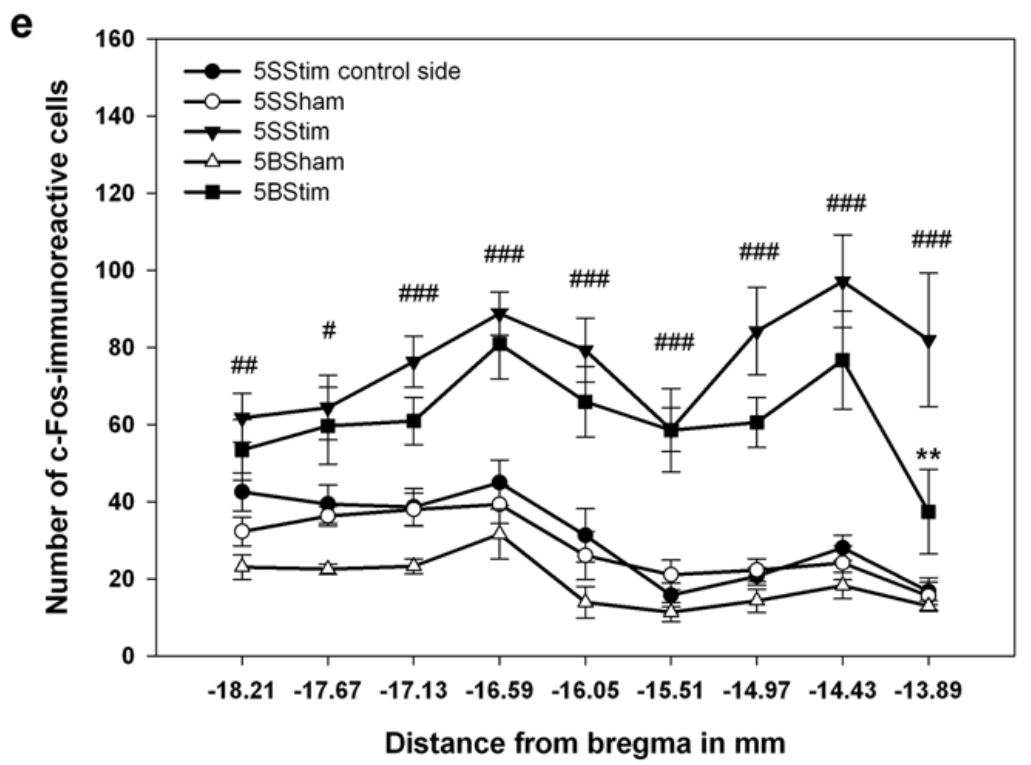

Figure 5.

Summary of the results from the mild stimulation paradigm regarding c-Fos immunostaining. Representative photos from the right sides of the four treatment groups after c-Fos immunohistochemistry, taken at $16.05 \mathrm{~mm}$ caudally from bregma: (a) 5SSham, (b) 5SStim, 
(c) 5BSham, (d) 5BStim. Scale bar $200 \mu \mathrm{m}$. Diagram showing the number of c-Fosimmunoreactive cells across the different levels of the TNC after mild electrical stimulation of the trigeminal ganglion (group means \pm SEM) (e). There was no significant difference between the control (left) sides (data not shown). For clarity, only the control side of the saline-treated stimulated group is presented. The hashmarks indicate significance in the comparison of the right sides of the 5SSham and 5SStim groups at different levels of the TNC $(\# \mathrm{p}<0.05 ; \# \# \mathrm{p}<0.01 ; \# \# \mathrm{p}<0.001)$. BBG treatment showed an attenuating tendency, though it proved to be significant merely at the level of $13.89 \mathrm{~mm}(* * \mathrm{p}<0.01,5 \mathrm{SStim}-5$ BStim).

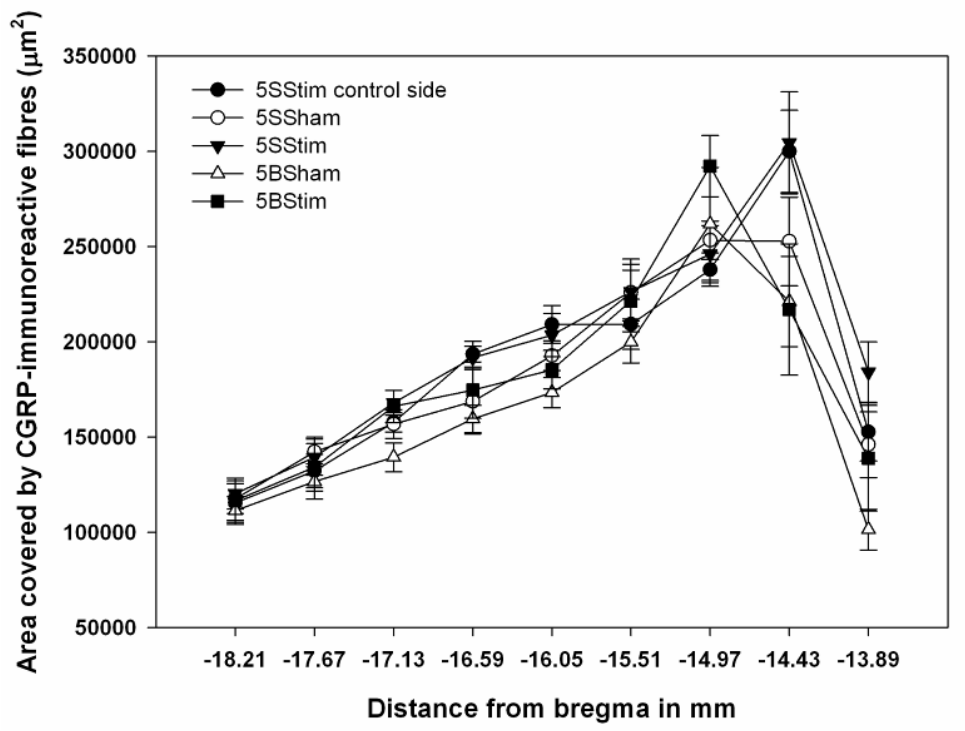

Figure 6.

Diagram showing the area covered by CGRP-immunoreactive fibres at the different levels of the TNC in the different treatment groups after mild electrical stimulation of the trigeminal ganglion (group means \pm SEM). There was no significant difference between either the control or the stimulated sides or the different groups. For clarity, only the control side of the saline-treated stimulated group is presented.

\section{Robust stimulation procedure}

A significant interaction was not found between the investigated factors of levels and groups for the number of c-Fos-immunoreactive cells in the robust stimulation paradigm. The levels had a significant effect $(\mathrm{p}<0.001)$, and there was also a significant difference between the groups $(\mathrm{p}<0.001)$. Pair wise comparisons revealed that there was no significant difference between the control sides of the four treatment groups (data not shown). Robust stimulation 
caused a marked increase in the number of c-Fos IR cells in the saline-treated animals (Fig 7 a, b, e, 30SStim compared with 30SSham). BBG had a significant attenuating effect on this increase (Fig 7 b, d, e, 30SStim compared with 30BStim).

a

\section{C}

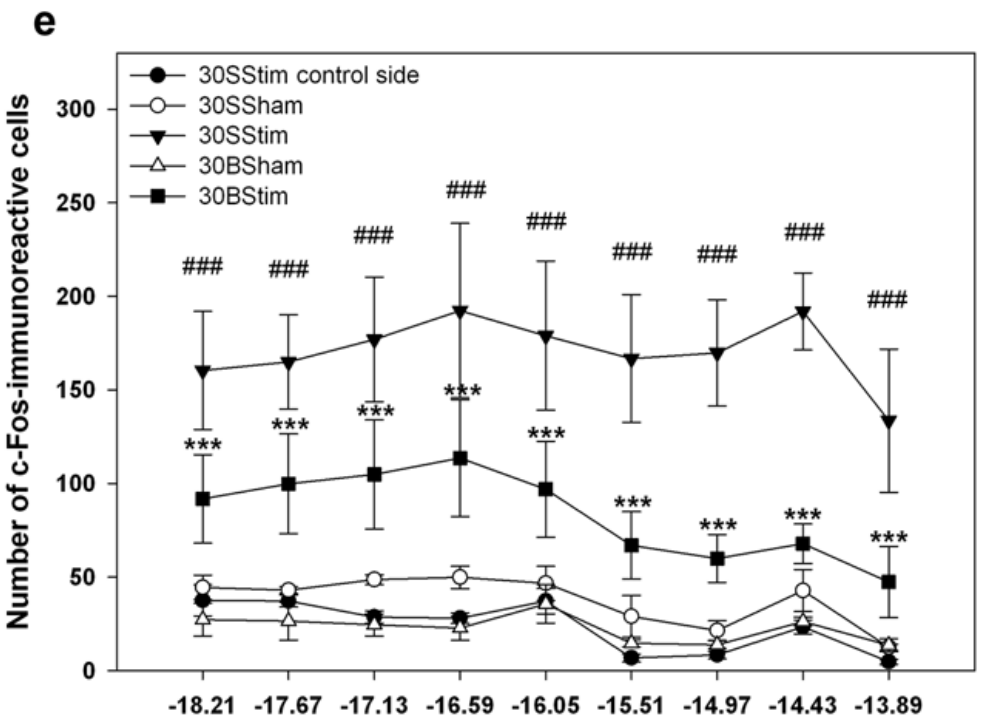

Figure 7. 
Summary of the results from the robust stimulation paradigm regarding c-Fos immunostaining. Representative photos from the right sides of the four treatment groups after c-Fos immunohistochemistry, taken at $16.05 \mathrm{~mm}$ caudally from bregma: (a) 30SSham, (b) 30SStim, (c) 30BSham, (d) 30BStim. Scale bar $200 \mu \mathrm{m}$. Diagram showing the number of cFos-immunoreactive cells at different levels along the rostro-caudal axis in the TNC (group means \pm SEM) in the robust stimulation paradigm (e). There was no significant difference between the control sides (data not shown). For clarity, only the control side of the salinetreated stimulated group is presented. Electrical stimulation of the right trigeminal ganglion caused a significant increase in the number of c-Fos-positive cells as compared with the right side of the sham group at all levels examined (\#\#\#p<0.001 30SSham - 30SStim). BBG treatment had a significant decreasing effect on the cell counts $(* * * p<0.00130$ SStim30BStim).

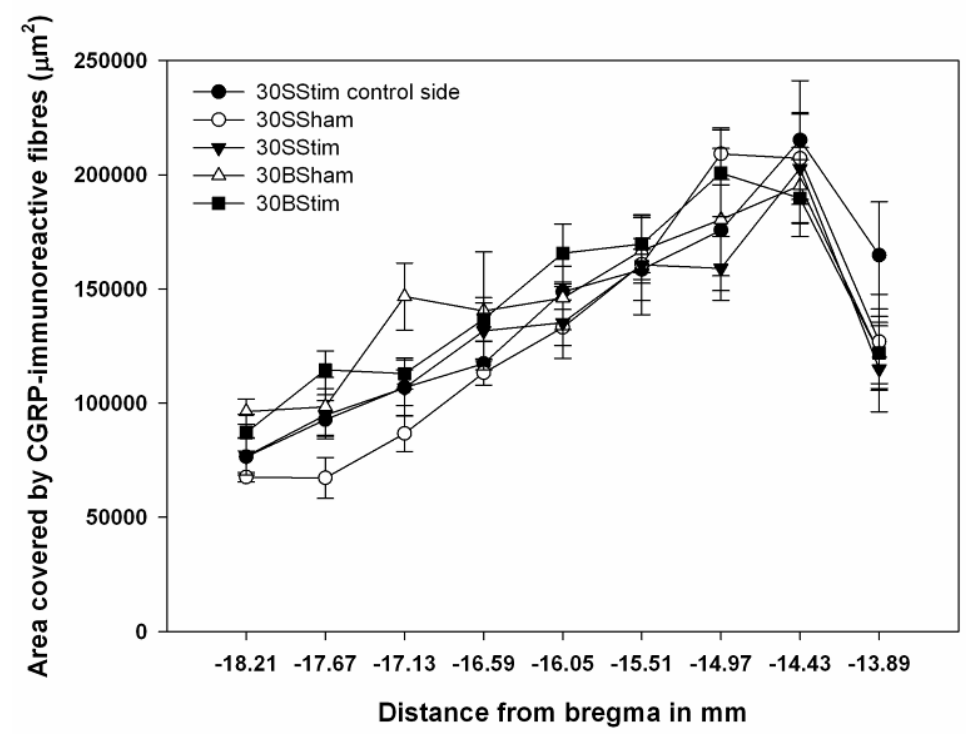

Figure 8.

Diagram showing the area covered by CGRP-immunoreactive fibres at the different levels of the TNC in the different treatment groups after robust electrical stimulation of the trigeminal ganglion (group means \pm SEM). There was no significant difference between either the control or the stimulated sides or the different groups. For clarity, only the control side of the saline-treated stimulated group is presented. 
CGRP expression was not altered by stimulation or BBG administration in any of the animal groups in the robust stimulation setup (Fig 8).

\section{Orofacial formalin test}

A significant interaction was found between time and groups $(p<0.05)$ during the analysis of the behavioural data from the orofacial formalin test. The pair wise comparison revealed that the nociceptive scores of the saline-injected groups (SSal and BSal) did not differ from each other at any time point (Fig 9). The injection of formalin caused a significant increase in the nociceptive scores in blocks 1 and 5-7 (Fig. 9, SForm compared with SSal). After the injection of formalin into the whisker pad, the BBG-treated animals demonstrated significantly increased nociceptive scores in blocks 1, 6 and 8 as compared with the control (Fig 9). The BBG-treated animals spent less time rubbing their formalin-injected side in both blocks 1 and 5-7, but this difference was not significant compared with the SForm group. In our experiments, the second phase of the formalin test subsided more quickly as expected in the SForm group, while it was more prolonged in the BForm group.

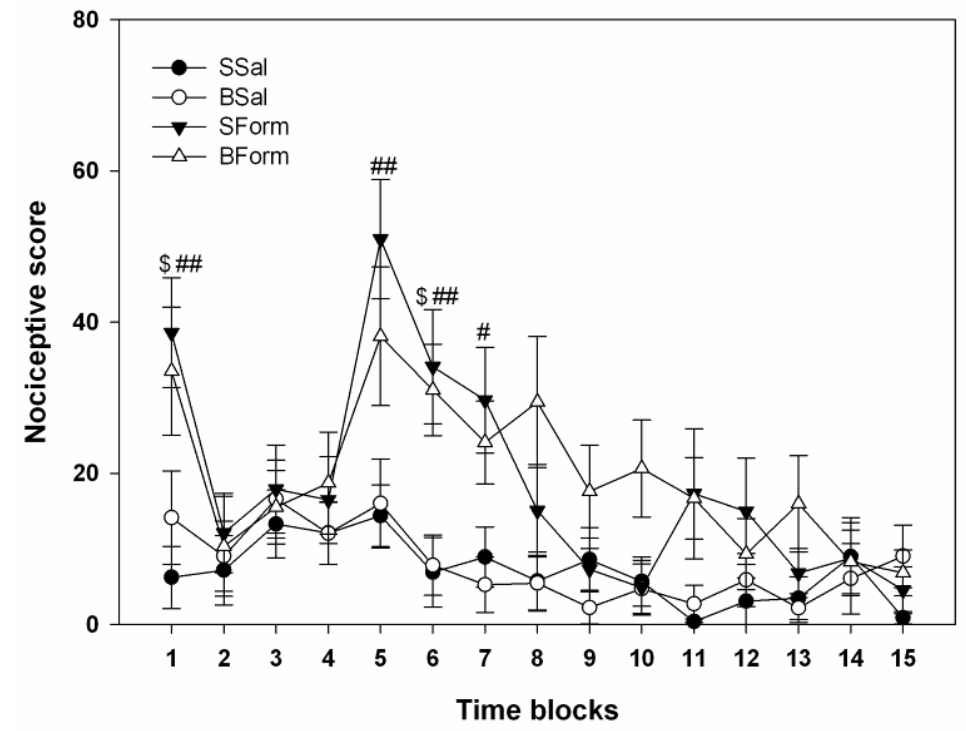

Figure 9.

Diagram showing the mean nociceptive scores $(\mathrm{sec})$ in different time blocks from the four treatment groups (group means \pm SEM, $n=13$ ) in the orofacial formalin test. The injection of formalin increased the nociceptive score in blocks 1 and 5-7 as compared with the saline injected animals $(\# p<0.05, \# \# p<0.01)$. BBG treatment did not affect the nociceptive scores 
after saline injection, and did not modify the normal behaviour. The nociceptive scores of the BBG and saline-treated formalin-injected groups did not differ significantly in any block.
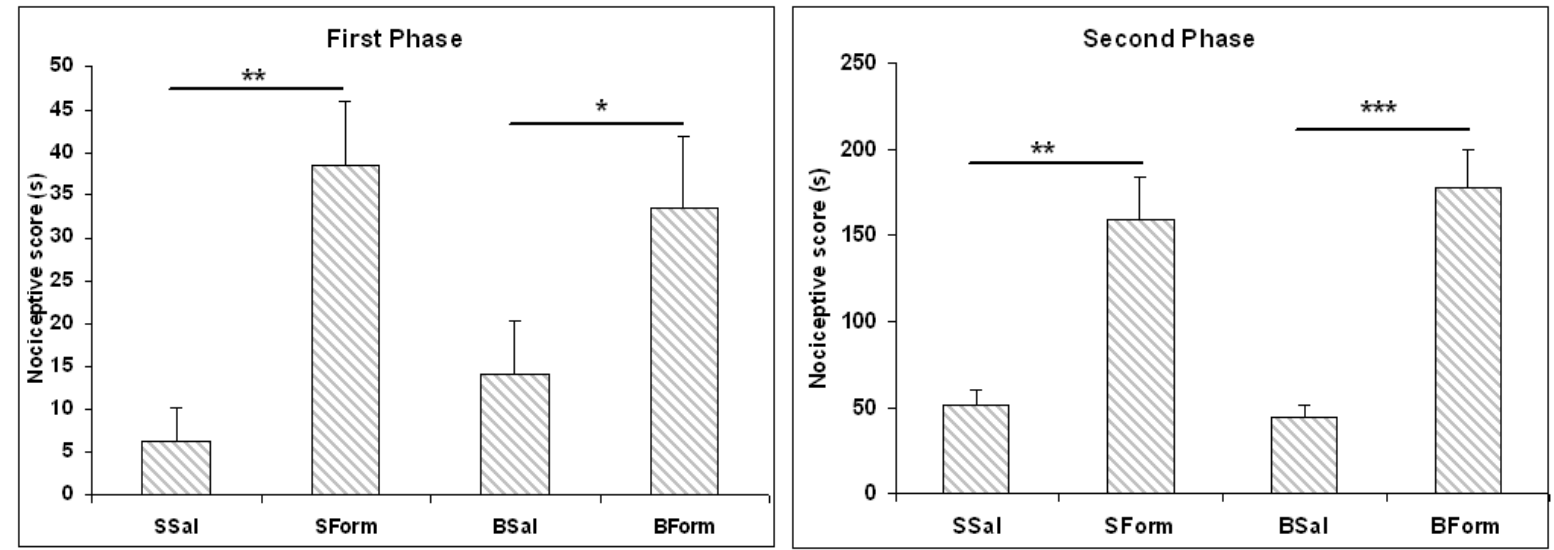

Figure 10.

Diagrams showing the nociceptive scores in the first (first block) and in the second (5-11 blocks) phases of the formalin test. Formalin raised the rubbing activity in the saline treated group. BBG administration did not show any modulating effect on the nociceptive score raised by formalin $(* \mathrm{p}<0.05 ; * * \mathrm{p}<0.01 ; * * * \mathrm{p}<0.001)$.

The two phases of the formalin test were distinguishable in our experiments, so we examined the data not just block-by-block, but also in the two phases separately. In the first phase of the formalin test an increase in the nociceptive scores can be seen in both the SForm and BForm groups. In the second phase a similar pattern could be observed, showing more clearly the lack of effect of the BBG treatment on formalin induced nociceptive behaviour. 
a

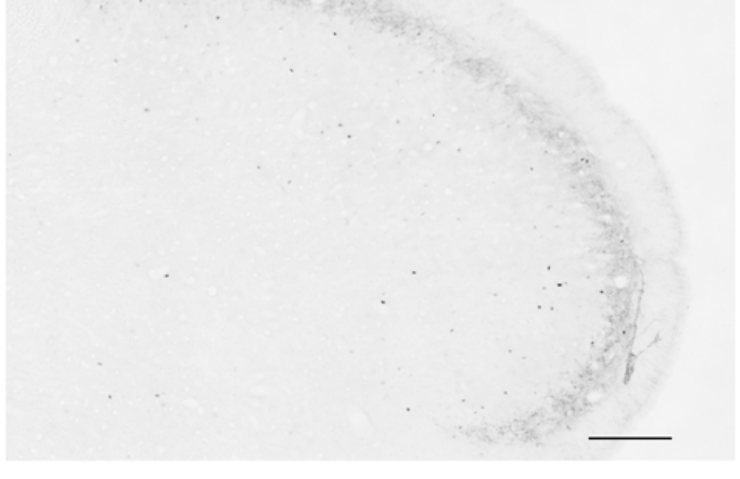

C

e

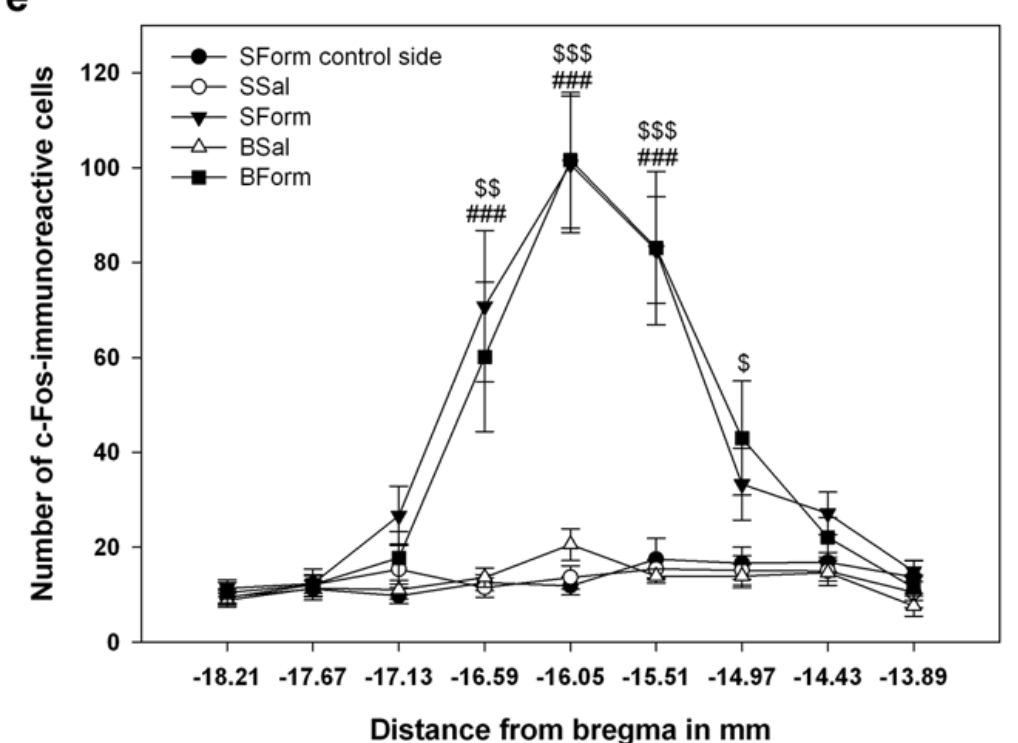

Figure 11.

Summary of the results from the orofacial formalin test regarding c-Fos immunostaining. Representative photos from the right (injected) sides of the four treatment groups after c-Fos immunohistochemistry taken at $16.05 \mathrm{~mm}$ caudally from bregma: (a) SSal, (b) SForm, (c) 
BSal, (d) BForm. Scale bar $200 \mu \mathrm{m}$. Diagram showing the mean number of c-Fos immunoreactive cells along the rostro-caudal axis in the TNC in the orofacial formalin test (group means \pm SEM) (e). Injection of saline did not cause any increase in the number of cFos-positive cells (SSal and BSal groups). The effect of formalin was visible only on the injected side; the control sides were similar to those in the saline-injected controls. For clarity, only the control side of the SForm animals is presented. Formalin caused an increase in c-Fos cell number on the ipsilateral side as compared with the saline-injected side of the controls (SForm compared with SSal), significant at the levels -16.59 to $-15.51 \mathrm{~mm}(\# \# \mathrm{p}<0.001)$. In the BBG-treated rats, formalin had a similar activity-increasing effect, significant at the levels -16.59 to $-14.97 \mathrm{~mm}(\$ \mathrm{p}<0.05 ; \$ \$ p<0.01 ; \$ \$ \$ p<0.001)$. The groups SForm and BForm did not differ significantly from each other.

A significant interaction was observed between the levels and groups $(p<0.001)$ for the number of c-Fos IR cells in the orofacial formalin test. There was no significant difference when either the control (left) sides or the control and saline-injected sides were compared at any level (Fig 11 e). The injection of formalin increased the number of c-Fos IR cells significantly at the levels $-16.59 \mathrm{~mm}$ to $-15.51 \mathrm{~mm}$, mainly in the central part of the TNC as compared with the saline-injected side in the SSal group. In the BForm group, a similar pattern was observed, except that the difference involved one additional level $(-14.97 \mathrm{~mm}$, Fig 11 e). There was no significant difference between the SForm and BForm groups at any level (Fig 11 b, d).

No group difference was found in any of the measured parameters regarding the CGRP immunoreactivity (Fig 12). 

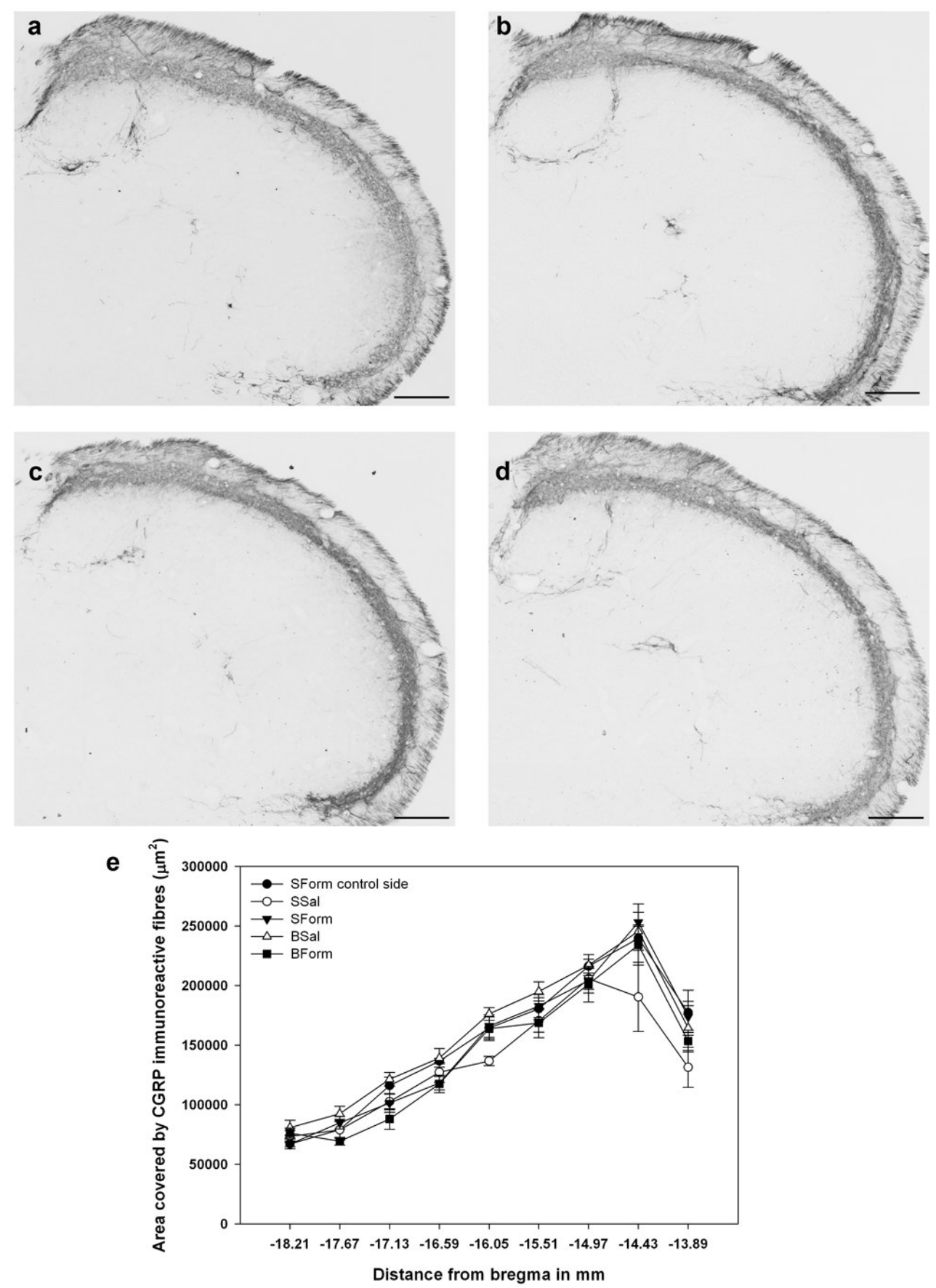

Figure 12.

Summary of the results after CGRP immunostaining in the orofacial formalin test. Representative photos of CGRP immunohistochemistry on the right sides of the TNC from the four treatment groups taken at $14.97 \mathrm{~mm}$ caudally from bregma (a) 5SSham, (b) 5SStim, (c) 5BSham, (d) 5BStim. Scale bar $200 \mu \mathrm{m}$. Diagram showing the area covered by CGRPimmunoreactive fibres at the different levels of the TNC in the different treatment groups in 
the orofacial formalin test (group means \pm SEM) (e). There was no significant difference between either the control or injected sides or the different groups. For clarity, only the control side of the saline-treated formalin-injected group is presented.

\section{Effects of probenecid after trigeminal activation}

The behavioural pattern observed in our experiments was in accordance with previous findings, as animals showed increased grooming activity after the formalin injection. We found significant differences in the first and fifth to eleventh blocks (Fig 13) between the Placebo-Phys and Placebo-Form groups. The pre-treatment with PROB decreased the formalin-induced nociceptive behaviour significantly in each block (Fig 13). However, PROB pre-treatment did not abolish the effect of formalin completely in the first block, where the time spent on rubbing was still significantly higher than that in the Placebo-Phys group (Fig 13). There was no significant difference between the Placebo-Phys and PROB-Phys animals in any block, nor was between the PROB-Form and PROB-Phys subgroups in any other block apart from the first (Fig 13).

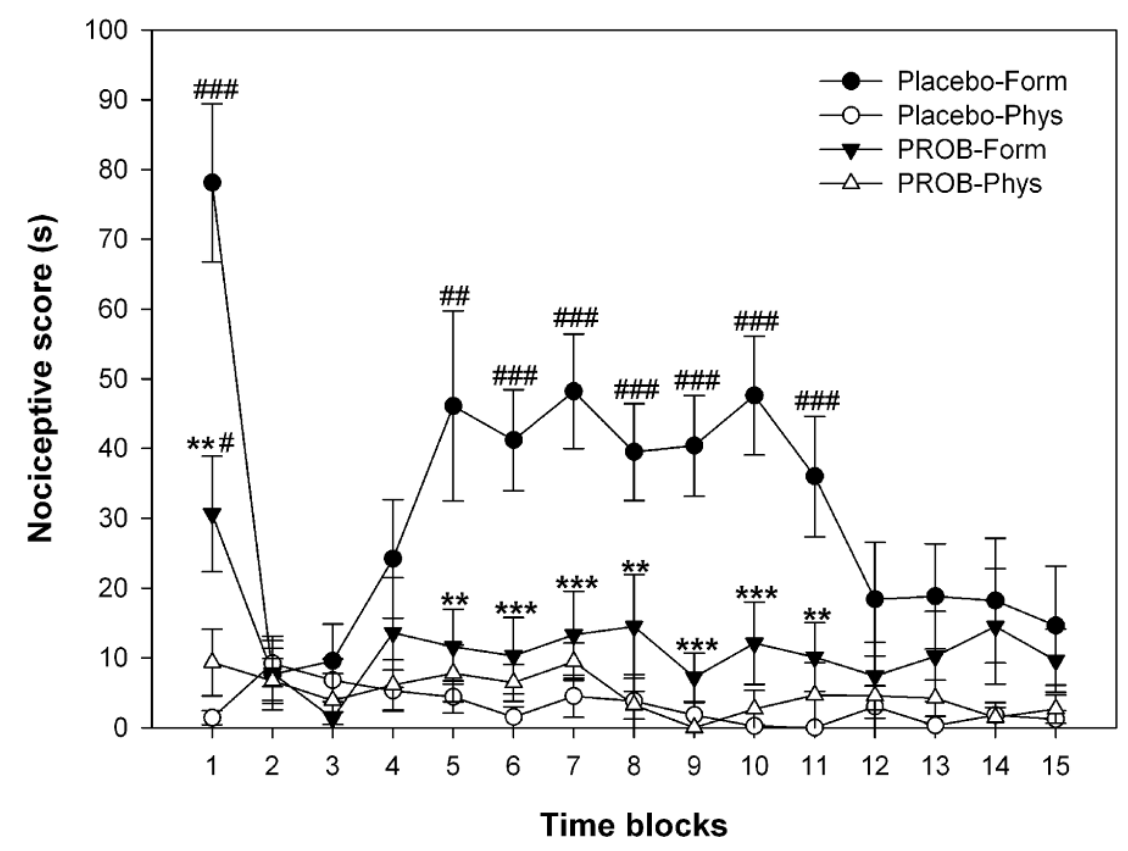

Figure 13.

Diagram showing the number of seconds spent rubbing the injected area with the ipsilateral fore- or hindpaw in each block. In the Placebo-Form group the two phases of formalin action are clearly distinguishable. The rubbing activity in the first $(\# \# \# p<0.001)$ and in the fifth to 
eleventh $(\# \# \mathrm{p}<0.01 ; \# \# \# \mathrm{p}<0.001)$ blocks was significantly higher in the Placebo-Form group than in the Placebo-Phys group. The PROB pre-treatment significantly reduced the formalin-induced nociceptive behaviour in each of the above-mentioned blocks $(* * p<0.01$; *** $\mathrm{p}<0.001)$ in the PROB-Form group compared to the Placebo-Form group. There was no significant difference between the nociceptive scores on the Placebo-Phys and PROB-Phys animals in any block.

Since the two phases of the formalin test were clearly distinguishable, we also examined the nociceptive scores of the two phases separately. The nociceptive score in the Placebo-Form group was significantly higher during both the first and the second phase than that in the Placebo-Phys group (Fig 14). PROB significantly decreased the nociceptive behaviour in both phases (Fig 14).
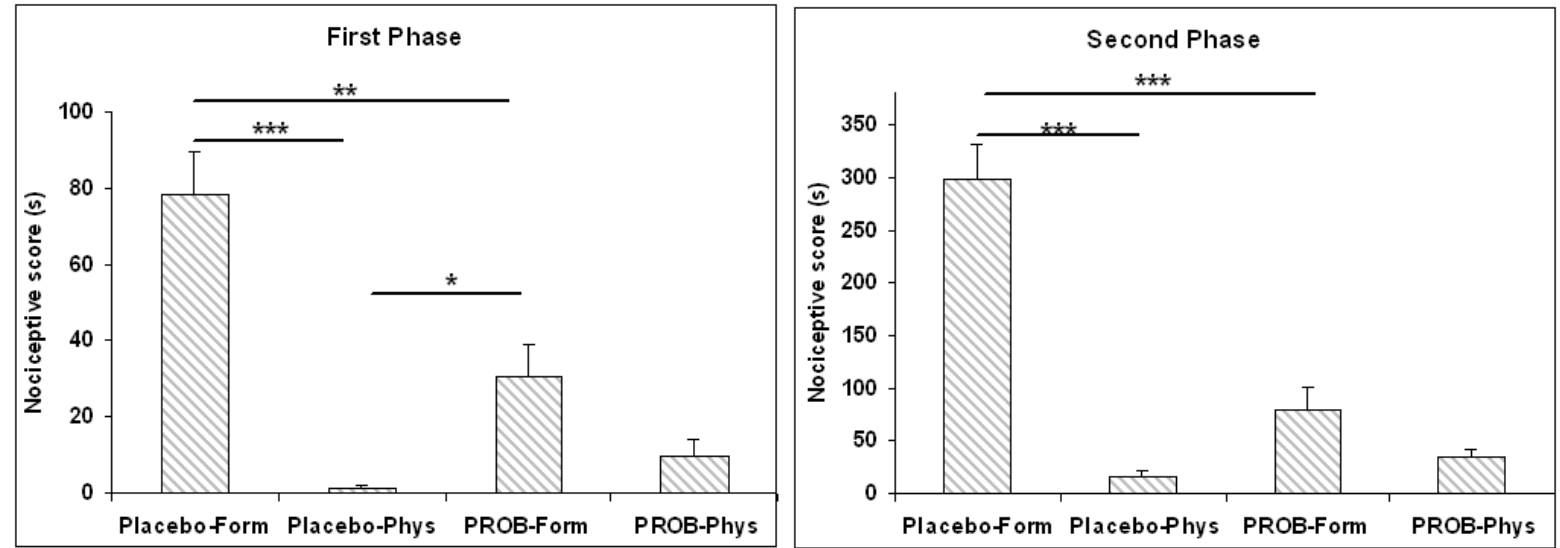

Figure 14.

Diagrams showing the nociceptive scores in the first and the second phase. In the Placebo group, the formalin injection (Placebo-Form) induced a significant increase in rubbing activity in both the first and the second phase $(* * * p<0.001)$ as compared with the salinetreated animals (Placebo-Phys). In both phases, pre-treatment with PROB had a significant effect on mitigating the formalin-induced nociceptive behaviour $(* * p<0.01 ; * * * p<0.001)$ as compared with the Placebo-Form group.

C-Fos immunohistochemistry revealed that in the Placebo-Form group, unilateral formalin injection produced an increase in the number of c-Fos-IR neurones in the dorsal, superficial area of the transverse sections of the ipsilateral TNC as compared with the nontreated contralateral side (Fig 15 A). This increase was significant at different levels along the 
rostrocaudal axis (between -0.3 and $-3.3 \mathrm{~mm}$ from the beginning of the TNC), in accordance with the somatotopic representation of the injected area (Fig 15 C).

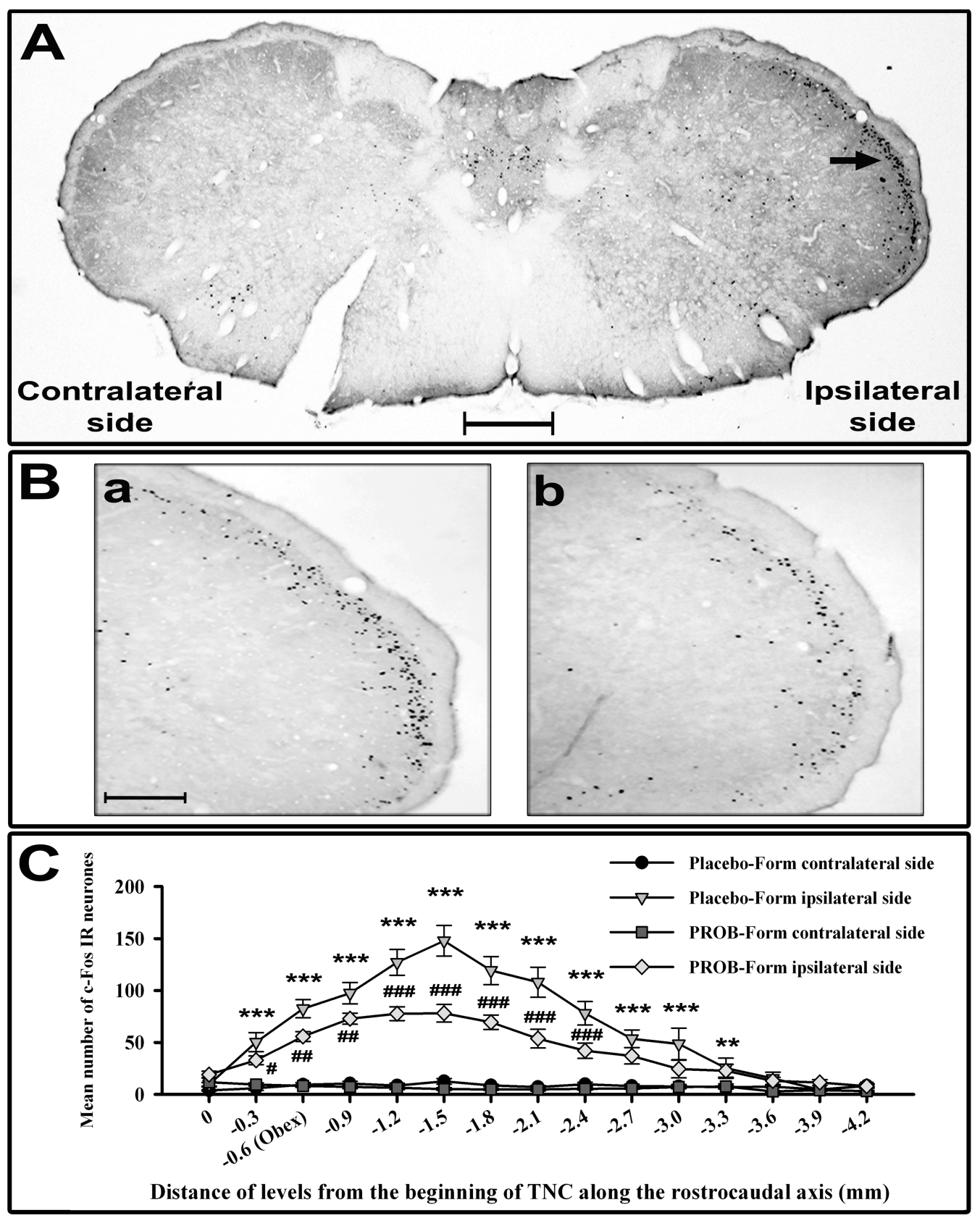

Figure 15.

C-Fos-IR neurones (black arrow) in the superficial laminae of a transverse section of the TNC in the Placebo-Form group (A). Formalin injection resulted in a higher number of c-Fos-IR neurones on the ipsilateral side compared to the contralateral side. Scale bar: $500 \mu \mathrm{m}$. (B) cFos-IR neurones on the formalin-treated ipsilateral sides of sections in the TNC in the Placebo-Form and PROB-Form groups. The formalin-induced increase in IR cells was more prominent in the Placebo-Form group $(\mathrm{B} / \mathrm{a})$ than in the PROB $(\mathrm{B} / \mathrm{b})$ pretreated animals. Scale 
bar: $200 \mu \mathrm{m}$. (C) Diagram showing the mean number of c-Fos IR cells in the superficial laminae of the TNC at different levels along the rostrocaudal axis (mean \pm S.E.M, $n=9$ per group). In the Placebo-Form group, formalin produced a significantly higher number of cFos-IR neurones on the formalin-treated side of the TNC as compared with the contralateral side at levels between -0.3 and $-3.3 \mathrm{~mm}$, in accordance with the somatotopic representation $(* * \mathrm{p}<0.01 ; * * * \mathrm{p}<0.001)$. PROB pre-treatment significantly decreased the effect of formalin between -0.3 and -2.4 mm (\#p < 0.05; \# \# < 0.01; \#\# \# $<0.001)$.

In the PROB-Form group, the number of c-Fos-IR neurones at the different levels along the rostrocaudal axis also increased in the ipsilateral TNC. This effect was similar, but less pronounced than that in the Placebo-Form group (Fig 15 B), i.e. PROB significantly decreased the formalin-related activation of the second order trigeminal neurones at several levels of the TNC (between -0.3 and $-2.4 \mathrm{~mm}$; Fig. $15 \mathrm{C}$ ). On the contralateral sides of the TNCs, no significant differences were noted either between the groups or between the different levels along the rostrocaudal axis (Fig 15).

The nNOS immunohistochemistry resulted in neurones showing cytoplasmic and dendritic staining. The formalin injection gave rise to an increase in the number of nNOS-IR neurones in the superficial area of the ipsilateral TNC compared to the non-treated contralateral side in the Placebo-Form group (Fig $16 \mathrm{~A}$ ). Along the rostrocaudal axis, significant increase can be observed at different levels (between -2.1 and -2.7 and between 3.3 and $-3.9 \mathrm{~mm}$; Fig 16 C). In the PROB-Form group, there was no difference in the number of nNOS-IR neurones between ipsilateral and contralateral TNC (Fig 16 B), PROB significantly decreased the number of the second order trigeminal neurones expressing nNOS at several levels of the TNC after formalin injection (between -2.1 and -2.7 and between -3.3 and $-3.9 \mathrm{~mm}$; Fig $16 \mathrm{C}$ ). On the contralateral sides of the TNCs, no significant differences were noted either between the subgroups or between the different levels along the rostrocaudal axis (Fig 16).

The Western blot analysis of the TNC segments did not show significant difference between the contra- and ipsilateral sides for any of the groups in IL-1 $\beta$ (data not shown), which means that IL-1 $\beta$ expression did not change four hours after the formalin injection and PROB pre-treatment did not have any modulatory effect on the expression of IL-1 $\beta$. 


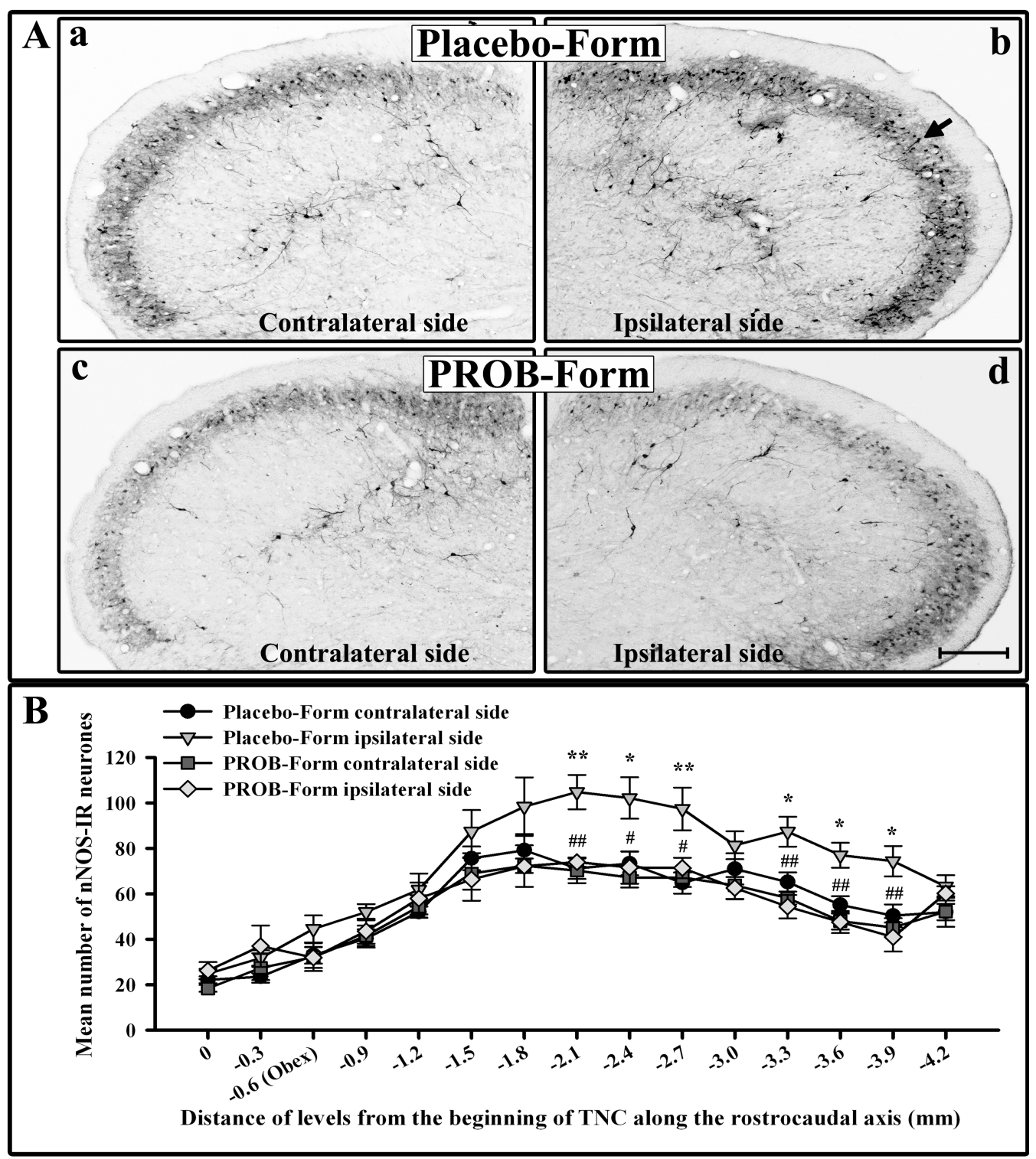

Figure 16.

nNOS immunostained sections of the TNC (A/a;b Placebo-Form; A/c;d PROB-Form). Black arrow shows a nNOS IR neurone in the TNC. More nNOS-IR neurones can be observed on the ipsilateral side $(\mathrm{A} / \mathrm{b})$, than on the contralateral side $(\mathrm{A} / \mathrm{a})$ in the Placebo-Form group. Comparing the ipsilateral sides of the two groups, fewer nNOS-IR cells can be found in the PROB-Form group ((A/b);(A/d)). There is no difference between the contralateral sides. Scale bar: $200 \mu \mathrm{m}$. (B) Diagram showing the mean number of nNOS-IR cells in the TNC at different levels along the rostrocaudal axis (mean \pm S.E.M). Formalin produced a significant increase in number of nNOS-IR neurones on the formalin-treated side as compared with the contralateral side at different levels (between -2.1 and -2.7 and between -3.3 and $-3.9 \mathrm{~mm}$; *p $<0.05 ; * * p<0.01)$. The effect of formalin was significantly decreased by pre-treatment with PROB between -2.1 and -2.7 and between -3.3 and -3.9 mm $(\# \mathrm{p}<0.05$; \# $\#<0.01)$. There was no significant difference between the contralateral sides. 


\section{Discussion}

ESTG has a direct effect on the primary trigeminal sensory neuron causing alterations in both the peripheral and the central endings. In the periphery, mediators from the nerve terminals around the meningeal vessels are released, which results in plasma protein extravasation and eventually neurogenic inflammation (15). In the central arm, there is a marked activation of the second-order neurones in the TNC (90). Our results are in accordance with these previous findings as we found marked increase in the number of c-Fos IR cells in the ipsilateral TNC both two and four hours after stimulation. This pronounced increase may arise directly from the electrical stimulation, or is a secondary phenomenon originating from the periphery due to the triggered dural extravasation and inflammation (15).

We were interested in the activation pattern of the migraine generator nuclei after ESTG, to examine if this model is suitable for mimicking the functional pattern of these nuclei observed in human spontaneous migraine attacks.

We found a significant increase in neuronal activity in the NRM in the two hour survival group, which persisted even four hours after the stimulation. Direct TNC projections to the NRM are sparse (91), and the superficial laminae, which are mainly activated in response to electrical stimulation, display even fewer connections to the NRM (92); accordingly activation of the TNC may not evoke a direct NRM activation. This hypothesis is further supported by the fact that we did not find any correlation between the increased cell activities in the two nuclei neither after 2 nor after 4 hours, suggesting that the activation of the NRM may not be a direct consequence of the activation of the TNC. Although, there is no correlation between the activation patterns of these two nuclei, the increase in their activity is present simultaneously at both 2 and 4 hours after stimulation.

The NRM is thought to be the main output of the descending pain modulatory system, providing dense innervations to the spinal cord dorsal horn and to the spinal trigeminal nuclei. We assume that the increased c-Fos IR of the NRM may be a consequence of the activation of this system, and could result from the cortical and thalamic input of the NRM, or it could originate from the PAG (91).

No change in the number of c-Fos immunoreactive nuclei was noticed in any region of the PAG. There is a direct connection between the TNC and the PAG, thus it is surprising that 
a strong stimulus like we applied caused no alteration in this area. Previous studies indicated an increase in activity of the PAG after nociceptive stimulation (93), although the stimulation parameters and settings differed from those we applied.

Activity of the cells in the LC did not change, however the activity of this nucleus was already pronounced in the sham group two hours after the stimulation, suggesting that the surgical procedure itself may obscure the changes caused by the electrical stimulation. After four hours of survival the c-Fos IR level of the LC is decreased both in the sham and stimulated groups, indicating that the activation of the LC is due to the invasive procedure.

There is no evidence that the TNC projects directly to the DR (94). The DR forms part of the pain modulatory system (37), and receives dense afferents from the NRM, but cortical input can also modulate the activity of this nucleus (94). We did not find significant change in the number of c-Fos IR cells in the DR after ESTG. Our results suggests that the activation of the trigeminal system does not cause the activation of the DR, thus in the rat this nucleus may not participate in the short-term regulation of trigeminal nociceptive processing.

If it is assumed that the changes seen in our experiments are secondary, the lack of direct projections from the TNC to most of the above-mentioned nuclei supports this hypothesis. In this concept, the stimulation activates the ascending nociceptive routes to the thalamus and to the cortex, and these structures react to the nociceptive input by activating the descending modulatory system. After trigeminal stimulation, the examined nuclei failed to exhibit the uniform activation detected during a migraine attack suggesting that this activation pattern of the migraine generator nuclei may be exclusively present in migraine.

Numerous stimulation parameters and stimulation times have been applied in previous experiments, and we therefore decided to make use of two stimulation procedures, a short, mild stimulation and a longer, robust stimulation, in order to examine the possible effects of P2X7-R antagonism on trigeminal activation. As P2X7-R blockade was previously found to be effective in inflammatory conditions, we additionally examined the effects of BBG in a model of orofacial inflammation, the orofacial formalin test.

Mild, short ESTG has been reported to lead to activation of the trigeminal system (54), and this was supported by our results.

We observed a similar pattern in the robust stimulation paradigm, the number of c-Fos IR cells increasing profoundly in the TNC, indicating the activation of the trigeminal system. 
The main difference seen between the two paradigms was in the number of cells activated after stimulation. Following the robust stimulation procedure more c-Fos IR cells were found in the TNC, suggesting a higher degree of activation than in the mild stimulation procedure. This increased activation may be attributed to the higher frequency applied in the robust paradigm, which can lead to the more rapid firing of the primary trigeminal neurons. An increased firing rate may cause elevated levels of transmitter release at both central and peripheral terminals, resulting in a higher degree of activation at the TNC level (87). It is also plausible that the longer stimulation interval leads to more primary cells being activated in the trigeminal ganglion, and hence in the TNC. We assume that in our experimental setting both the increased frequency and the increased stimulation interval contributed to the higher activation level in the TNC.

Pre-treatment with the P2X7-R antagonist BBG was effective only in the robust stimulation paradigm, resulting in a decrease of the activity, reflected by the c-Fos expression. P2X7-Rs can be found in the trigeminal system, both in the ganglion (95) and in the TNC (96), therefore BBG may modulate peripheral and central processes. BBG could modulate the nociceptive processing by interfering with the peripheral neurogenic inflammation, or by modulating non-synaptic communication within the ganglion (97). At the central level, BBG could affect P2X7-Rs on central presynaptic terminals and modulate glutamate release (96), and thereby influence nociceptive transmission. Presumably, after the robust stimulation, where the more pronounced peripheral activation and more severe inflammation also involve P2X7-Rs, the blocking effect of BBG manifests, while in the mild paradigm due to the minor changes the effect of BBG does not emerge.

Neither the mild nor the robust stimulation procedure caused alterations in the levels of CGRP. It was earlier found that ESTG with parameters similar to our robust stimulation, led to the depletion of CGRP from the medial one-third of the central terminals of the trigeminal afferents (98). However, those examinations were conducted immediately after stimulation of the trigeminal ganglion, whereas in our experiments a 2 or a 4 hour survival time was included for better observability of the activity changes (c-Fos). These periods might be sufficient for the depleted CGRP to be resynthesized and for the changes in CGRPimmunoreactivity seen immediately after stimulation to normalize. BBG treatment did not modify the levels of CGRP in either the sham or the stimulated group. 
The injection of formalin into the whisker pad causes a biphasic behavioural effect (56), as seen in our experiments also. BBG did not exhibit any effect in the first phase of the formalin response. When formalin was applied to the hind paw and was combined with a selective P2X7-R antagonist, A-438079, in previous work, protective effect was exerted only in the second phase of the formalin test (66). Furthermore, BBG was earlier shown to be hyperalgesic in the modulation of acute nociception in the hot-plate test (62). These results suggest that BBG and blockade of the P2X7-Rs may not be effective against acute nociception.

In the second phase of the formalin test, BBG did not demonstrate any obvious effect. At the beginning of the second phase (in blocks 5-7), the nociceptive scores revealed a decreasing tendency, while in the later blocks the opposite could be observed. Since another P2X7-R antagonist was effective when formalin was applied at the hind paws, our results suggest that the role of the P2X7-Rs in the sensory system is not uniform.

Four hours after formalin injection, c-Fos immunohistochemistry revealed that the TNC displays clear activation. The pattern of activation corresponds to the somatotopic projection pattern of the injected area. BBG had no effect on the activation of the trigeminal system after formalin. Our results in the orofacial formalin test are somewhat surprising, considering that other antagonists of the P2X7-Rs $(65,66,99)$ and even BBG (62) have proven effective in numerous inflammatory models. However, none of these experiments related to the trigeminal system, and our results are the first regarding the effects of blockade of the P2X7-Rs by BBG in this area after inflammation caused by formalin.

The levels of CGRP were not altered 4 hours after formalin injection, and following treatment with BBG. Alterations in CGRP usually occur immediately during or after the applied stimulus and cease within a matter of hours $(47,100)$, and our results are in line with this. The effect of P2X7-R antagonism on the expression of CGRP in the formalin test should be further elucidated with regard to the time scale.

BBG in the micromolar range was previously shown to inhibit voltage-dependent sodium channels in vitro (101), and it might therefore be possible that this feature of BBG contributes to its effects in our experiments. This is rather unlikely, considering that we applied BBG in a single dose, which has been shown not to reach micromolar levels even after continuous administration in mice (102). 
Our results suggest that $\mathrm{P} 2 \mathrm{X} 7-\mathrm{Rs}$ have a role in the modulation of trigeminal nociceptive processing. Further investigations of the relations of the trigeminal system and P2X7-R signalling may provide important details concerning trigeminal nociceptive functions and the pathomechanism of headaches.

In our experiments PROB produced a pronounced antinociceptive behavioural effect in the orofacial formalin test especially in the second phase. PROB exhibited no effect on the baseline activity of the control animals, which received a saline injection in the whisker pad. In addition to the modulation of the nociceptive behaviour, PROB also mitigated the formalin induced c-Fos and nNOS expression in the TNC - considered markers of activation and sensitization of these neurons $(103,104)$.

Previously PROB proved to be effective also after systemic nitroglycerin administration, decreasing the levels of sensitization markers and modulating CGRP levels in the TNC (105). The antinociceptive effect of PROB along with its inhibitory effect on the trigeminal activation and sensitization can be the result of various mechanisms:

PROB inhibits MRP4 (75), which releases key inflammatory mediators such as PGE $_{1}$ and $\mathrm{PGE}_{2}$ from cells in a time- and ATP-dependent manner (76). $\mathrm{PGE}_{2}$ can sensitize transient receptor potential channel subtype V member 1 (TRPV1) (106), a key factor in the development of sensitization during nociception (107). Moreover, $\mathrm{PGE}_{2}$ can induce CGRP release from cultured trigeminal ganglion cells (108). $\mathrm{PGE}_{2}$ can also act on the central site of the trigeminal system by enhancing the capsaicin-induced CGRP release in slice preparations of the TNC (109). The expression of cyclooxygenase-2, the inducible enzyme of prostaglandin synthesis (110) has also been detected in the neurones and glial cells in the trigeminal ganglion (111) and in the neurones of the TNC (112). In addition, prostaglandin receptors are present in the trigeminal ganglion (113), co-expressed with TRPV1, with a functional interaction between them in the trigeminal system $(114,115)$, and are involved in mediating CGRP release from cultured rat trigeminal neurones (116). Quantitative autoradiography has revealed that $\mathrm{TNC}$ has a moderate density of $\mathrm{PGE}_{2}$ binding sites as well (117). Accordingly, a possible mechanism of action of PROB is the inhibition of the release of $\mathrm{PGE}_{2}$, a key player in trigeminal nociception and an important molecule in migraine genesis (118) through the blockade of MRP4. 
Data suggest that PROB is able to activate some of the transient receptor potential channels, including TRPV2 and TRPA1 $(77,78)$ which play an important role in pain perception $(79,80)$ and are present in the trigeminal system $(119,120)$. However, the agonistmediated desensitization of these receptors is also known $(77,81)$, which can cause an antinociceptive effect $(121,122)$. PROB can desensitize the TRPA1 (77) and may act similarly on TRPV2 as well, which might account of its antinociceptive properties in the present experiments.

PROB also inhibits the PANX1 (82), which is expressed at several sites in the central nervous system (123); and is crucial in caspase-1 activation leading to the production and release of IL-1 $\beta$ in neurones, astrocytes and macrophages $(83,84)$. The release of IL-1 $\beta$ from trigeminal ganglion satellite cells may contribute to the mechanisms underlying trigeminal inflammatory hyperalgesia (124). Up-regulation of astrocytic IL-1 $\beta$ in the TNC can lead to central sensitization via its receptors present on trigeminal neurones in the same area $(125$, 126). These results clearly indicate that IL-1 $\beta$ plays a role in trigeminal activation. However the results of our Western blot measurement showed that there were no changes in the expression of IL-1 $\beta$ at the level of TNC four hours after formalin administration and suggesting that PROB may not influences IL-1 $\beta$ in the TNC, but one can not exclude the possibility that PROB affects IL- $1 \beta$ in the peripheral sites of the trigeminal system.

PROB can increase the concentration of kynurenic acid, an endogenous tryptophan metabolite (127) in the central nervous system (128). This phenomenon can be caused by inhibition of organic anion transporters by PROB $(129,130)$, which are involved in the transport of kynurenic acid from brain through the blood-brain barrier (131) or by increasing of concentration of tryptophan in the central nervous system (132). Experimental data suggest that kynurenic acid can affect nociception (133-135) and elevation in its concentration in the brain can inhibit the trigeminal activation $(136,137)$, probably due to an antagonistic effect on ionotropic glutamate receptors (138) or due an agonistic action on G-protein-coupled receptor-35 (139). Therefore the antinociceptive effect of PROB may be related to the increases in kynurenic acid concentration. 


\section{Conclusions}

ESTG caused clear activation only in the NRM, not in the other migraine generator nuclei. This activation pattern does not correspond to what was seen in migraine patients during the attack. The origin of the brainstem activation is not known, it can be the consequence of pain, but the persistent activation of these structures after headache cessation suggests, that the activation of these nuclei is specific for migraine (18). Our result support this hypothesis, as the activation of the trigeminal system not resulted in the activation of the migraine generator nuclei. However, the exact mechanisms of brainstem activation in migraine patients needs to be further elucidated. Our result in the rat demonstrate that in this model the functioning of the descending pain modulatory system can be studied, and the further characterization of molecular mechanism in the rat may provide useful information about headache pathomechanism.

Blocking the P2X7-R by BBG was able to modulate trigeminal activation only after the robust stimulation, suggesting that the role of $\mathrm{P} 2 \mathrm{X} 7-\mathrm{Rs}$ in trigeminal nociception is complex, and may depend on the stimulus applied. Similar controversial effects were observed in an earlier work with BBG (62), supporting that a more detailed evaluation of $\mathrm{P} 2 \mathrm{X} 7$ receptor functioning in the trigeminal system is needed.

PROB showed clear antinociceptive effect in the formalin test, and also in previous experiments (105), however it is not clear which of its molecular targets is responsible for this effect.

Our result provide important information about the functioning of the trigeminal system, thus contribute to the understanding of pathological processes underlying headache and migraine. 


\section{Acknowledgements}

I would like to express my sincere gratitude to my supervisor and mentor Dr. Árpád Párdutz for the excellent guidance, and for forming my general view of scientific research since my graduate years. I cannot be grateful enough for his patience.

I would like to thank Professor László Vécsei for making it possible to do my research at his Department and showing me that unlimited enthusiasm about science exists.

I would like to give special thanks to my closest colleagues Dr. Annamária-Fejes Szabó and Gábor Nagy Grócz, whom I performed most of the experiments and experienced the challenges of scientific research.

I am obliged to Valéria Vékonyné Széll for showing me the basic standards of laboratory work, for helping me a lot during my years as a student and for helping me manage the everyday life in the lab.

I am also grateful for all my current and former colleagues who helped me prepare this work, especially to Dr. Hedvig Varga, Dr Enikő Vámos and to Dr. Bernadett Tuka.

I am deeply indebted to my family, especially to my parents and fiancé for supporting me during all these years and for believing in me. 


\section{REFERENCES}

[1] (IHS) HCCotIHS. The International Classification of Headache Disorders, 3rd edition (beta version). Cephalalgia. 2013;33(2013):629-808.

[2] Stovner LJ, Andree C. Prevalence of headache in Europe: a review for the Eurolight project. J Headache Pain. 2010;11(2010):289-99.

[3] Smitherman TA, Burch R, Sheikh H, Loder E. The prevalence, impact, and treatment of migraine and severe headaches in the United States: a review of statistics from national surveillance studies. Headache. 2013;53(2013):427-36.

[4] Linde M, Gustavsson A, Stovner LJ, Steiner TJ, Barré J, Katsarava Z, et al. The cost of headache disorders in Europe: the Eurolight project. Eur J Neurol. 2012;19(2012):703-11.

[5] Manzoni GC, Stovner LJ. Epidemiology of headache. Handb Clin Neurol. 2010;97(2010):3-22.

[6] Borsook D, Erpelding N, Lebel A, Linnman C, Veggeberg R, Grant PE, et al. Sex and the migraine brain. Neurobiol Dis. 2014;68(2014):200-14.

[7] Russell MB, Iselius L, Olesen J. Migraine without aura and migraine with aura are inherited disorders. Cephalalgia. 1996;16(1996):305-9.

[8] Persico AM, Verdecchia M, Pinzone V, Guidetti V. Migraine genetics: current findings and future lines of research. Neurogenetics. 2015;16(2015):77-95.

[9] Russell MB, Rasmussen BK, Fenger K, Olesen J. Migraine without aura and migraine with aura are distinct clinical entities: a study of four hundred and eighty-four male and female migraineurs from the general population. Cephalalgia. 1996;16(1996):239-45.

[10] Anttila V, Winsvold BS, Gormley P, Kurth T, Bettella F, McMahon G, et al. Genomewide meta-analysis identifies new susceptibility loci for migraine. Nat Genet.

2013;45(2013):912-7.

[11] Zhao H, Eising E, de Vries B, Vijfhuizen LS, Anttila V, Winsvold BS, et al. Genebased pleiotropy across migraine with aura and migraine without aura patient groups.

Cephalalgia. 2015(2015).

[12] Ray BS, Wolff HG. Experimental studies on headache: Pain-sensitive structures of the head and their significance in headache. Archives of Surgery. 1940;41(1940):813-56.

[13] Wolff HG. Wolff's Headache and other head pain. 3d ed. New York,: Oxford University Press, 1972.

[14] Edvinsson L, Villalón CM, MaassenVanDenBrink A. Basic mechanisms of migraine and its acute treatment. Pharmacol Ther. 2012;136(2012):319-33. 
[15] Markowitz S, Saito K, Moskowitz MA. Neurogenically mediated leakage of plasma protein occurs from blood vessels in dura mater but not brain. J Neurosci. 1987;7(1987):412936.

[16] Moskowitz MA. Neurogenic versus vascular mechanisms of sumatriptan and ergot alkaloids in migraine. Trends Pharmacol Sci. 1992;13(1992):307-11.

[17] Moskowitz MA. Neurogenic inflammation in the pathophysiology and treatment of migraine. Neurology. 1993;43(1993):S16-20.

[18] Weiller C, May A, Limmroth V, Jüptner M, Kaube H, Schayck RV, et al. Brain stem activation in spontaneous human migraine attacks. Nat Med. 1995;1(1995):658-60.

[19] Afridi SK, Giffin NJ, Kaube H, Friston KJ, Ward NS, Frackowiak RS, Goadsby PJ. A positron emission tomographic study in spontaneous migraine. Arch Neurol.

2005;62(2005):1270-5.

[20] Afridi SK, Matharu MS, Lee L, Kaube H, Friston KJ, Frackowiak RS, Goadsby PJ. A PET study exploring the laterality of brainstem activation in migraine using glyceryl trinitrate. Brain. 2005;128(2005):932-9.

[21] Denuelle M, Fabre N, Payoux P, Chollet F, Geraud G. Hypothalamic activation in spontaneous migraine attacks. Headache. 2007;47(2007):1418-26.

[22] May A, Kaube H, Buchel C, Eichten C, Rijntjes M, Juptner M, et al. Experimental cranial pain elicited by capsaicin: a PET study. Pain. 1998;74(1998):61-6.

[23] Basbaum AI, Fields HL. Endogenous pain control systems: brainstem spinal pathways and endorphin circuitry. Annu Rev Neurosci. 1984;7(1984):309-38.

[24] Chen T, Wang XL, Qu J, Wang W, Zhang T, Yanagawa Y, et al. Neurokinin-1 receptor-expressing neurons that contain serotonin and gamma-aminobutyric acid in the rat rostroventromedial medulla are involved in pain processing. J Pain. 2013;14(2013):778-92.

[25] Fields HL, Heinricher MM. Anatomy and physiology of a nociceptive modulatory system. Philos Trans R Soc Lond B Biol Sci. 1985;308(1985):361-74.

[26] Harasawa I, Johansen JP, Fields HL, Porreca F, Meng ID. Alterations in the rostral ventromedial medulla after the selective ablation of $\mu$-opioid receptor expressing neurons. Pain. 2016;157(2016):166-73.

[27] Li YQ, Takada M, Shinonaga Y, Mizuno N. Direct projections from the midbrain periaqueductal gray and the dorsal raphe nucleus to the trigeminal sensory complex in the rat. Neuroscience. 1993;54(1993):431-43.

[28] Heinricher MM, Cheng ZF, Fields HL. Evidence for two classes of nociceptive modulating neurons in the periaqueductal gray. J Neurosci. 1987;7(1987):271-8. 
[29] Behbehani MM. Functional characteristics of the midbrain periaqueductal gray. Prog Neurobiol. 1995;46(1995):575-605.

[30] McMahon SB. Wall and Melzack's textbook of pain. 6th ed. Philadelphia, PA: Elsevier/Saunders, 2013.

[31] Knight YE, Goadsby PJ. The periaqueductal grey matter modulates trigeminovascular input: a role in migraine? Neuroscience. 2001;106(2001):793-800.

[32] Samuels ER, Szabadi E. Functional neuroanatomy of the noradrenergic locus coeruleus: its roles in the regulation of arousal and autonomic function part I: principles of functional organisation. Curr Neuropharmacol. 2008;6(2008):235-53.

[33] Proudfit HK. Pharmacologic evidence for the modulation of nociception by noradrenergic neurons. Prog Brain Res. 1988;77(1988):357-70.

[34] Mokha SS, McMillan JA, Iggo A. Pathways mediating descending control of spinal nociceptive transmission from the nuclei locus coeruleus (LC) and raphe magnus (NRM) in the cat. Exp Brain Res. 1986;61(1986):597-606.

[35] Simpson KL, Altman DW, Wang L, Kirifides ML, Lin RC, Waterhouse BD.

Lateralization and functional organization of the locus coeruleus projection to the trigeminal somatosensory pathway in rat. J Comp Neurol. 1997;385(1997):135-47.

[36] Lance JW, Lambert GA, Goadsby PJ, Duckworth JW. Brainstem influences on the cephalic circulation: experimental data from cat and monkey of relevance to the mechanism of migraine. Headache. 1983;23(1983):258-65.

[37] Wang QP, Nakai Y. The dorsal raphe: an important nucleus in pain modulation. Brain Res Bull. 1994;34(1994):575-85.

[38] Oliveras JL, Guilbaud G, Besson JM. A map of serotoninergic structures involved in stimulation producing analgesia in unrestrained freely moving cats. Brain Res. 1979;164(1979):317-22.

[39] Mantyh PW, Peschanski M. Spinal projections from the periaqueductal grey and dorsal raphe in the rat, cat and monkey. Neuroscience. 1982;7(1982):2769-76.

[40] Kirifides ML, Simpson KL, Lin RC, Waterhouse BD. Topographic organization and neurochemical identity of dorsal raphe neurons that project to the trigeminal somatosensory pathway in the rat. J Comp Neurol. 2001;435(2001):325-40.

[41] Fields HL, Basbaum AI. Brainstem control of spinal pain-transmission neurons. Annu Rev Physiol. 1978;40(1978):217-48.

[42] Leao A. Spreading depression of activity in the cerebral cortex. J Neurophysiol. 1944;7(1944):359-90. 
[43] Hadjikhani N, Sanchez Del Rio M, Wu O, Schwartz D, Bakker D, Fischl B, et al. Mechanisms of migraine aura revealed by functional MRI in human visual cortex. Proc Natl Acad Sci U S A. 2001;98(2001):4687-92.

[44] Lauritzen M, Olesen J. Regional cerebral blood flow during migraine attacks by Xenon-133 inhalation and emission tomography. Brain. 1984;107 ( Pt 2)(1984):447-61.

[45] Olesen J, Burstein R, Ashina M, Tfelt-Hansen P. Origin of pain in migraine: evidence for peripheral sensitisation. Lancet Neurol. 2009;8(2009):679-90.

[46] Andres KH, von Düring M, Muszynski K, Schmidt RF. Nerve fibres and their terminals of the dura mater encephali of the rat. Anat Embryol (Berl). 1987;175(1987):289301.

[47] Buzzi MG, Carter WB, Shimizu T, Heath H, 3rd, Moskowitz MA. Dihydroergotamine and sumatriptan attenuate levels of CGRP in plasma in rat superior sagittal sinus during electrical stimulation of the trigeminal ganglion. Neuropharmacology. 1991;30(1991):1193200.

[48] Goadsby PJ, Edvinsson L, Ekman R. Release of vasoactive peptides in the extracerebral circulation of humans and the cat during activation of the trigeminovascular system. Ann Neurol. 1988;23(1988):193-6.

[49] Buzzi MG, Moskowitz MA. The antimigraine drug, sumatriptan (GR43175), selectively blocks neurogenic plasma extravasation from blood vessels in dura mater. $\mathrm{Br} \mathrm{J}$ Pharmacol. 1990;99(1990):202-6.

[50] Markowitz S, Saito K, Moskowitz MA. Neurogenically mediated plasma extravasation in dura mater: effect of ergot alkaloids. A possible mechanism of action in vascular headache. Cephalalgia. 1988;8(1988):83-91.

[51] Uhl GR, Walther D, Nishimori T, Buzzi MG, Moskowitz MA. Jun B, c-jun, jun D and c-fos mRNAs in nucleus caudalis neurons: rapid selective enhancement by afferent stimulation. Brain Res Mol Brain Res. 1991;11(1991):133-41.

[52] Knyihar-Csillik E, Tajti J, Mohtasham S, Sari G, Vecsei L. Electrical stimulation of the Gasserian ganglion induces structural alterations of calcitonin gene-related peptideimmunoreactive perivascular sensory nerve terminals in the rat cerebral dura mater: a possible model of migraine headache. Neurosci Lett. 1995;184(1995):189-92.

[53] Mitsikostas DD, Sanchez del Rio M. Receptor systems mediating c-fos expression within trigeminal nucleus caudalis in animal models of migraine. Brain Res Brain Res Rev. 2001;35(2001):20-35.

[54] Takemura M, Shimada T, Sugiyo S, Nokubi T, Shigenaga Y. Mapping of c-Fos in the trigeminal sensory nucleus following high- and low-intensity afferent stimulation in the rat. Exp Brain Res. 2000;130(2000):113-23. 
[55] Tjolsen A, Berge OG, Hunskaar S, Rosland JH, Hole K. The formalin test: an evaluation of the method. Pain. 1992;51(1992):5-17.

[56] Clavelou P, Pajot J, Dallel R, Raboisson P. Application of the formalin test to the study of orofacial pain in the rat. Neurosci Lett. 1989;103(1989):349-53.

[57] McCall WD, Tanner KD, Levine JD. Formalin induces biphasic activity in C-fibers in the rat. Neurosci Lett. 1996;208(1996):45-8.

[58] Hunskaar S, Hole K. The formalin test in mice: dissociation between inflammatory and non-inflammatory pain. Pain. 1987;30(1987):103-14.

[59] Wang LG, Li HM, Li JS. Formalin induced FOS-like immunoreactive neurons in the trigeminal spinal caudal subnucleus project to contralateral parabrachial nucleus in the rat. Brain Res. 1994;649(1994):62-70.

[60] Tomić MA, Pecikoza UB, Micov AM, Popović BV, Stepanović-Petrović RM. The effects of levetiracetam, sumatriptan, and caffeine in a rat model of trigeminal pain: interactions in 2-component combinations. Anesth Analg. 2015;120(2015):1385-93.

[61] Burnstock G. Purinergic mechanisms and pain--an update. Eur J Pharmacol. 2013;716(2013):24-40.

[62] Ando RD, Mehesz B, Gyires K, Illes P, Sperlagh B. A comparative analysis of the activity of ligands acting at $\mathrm{P} 2 \mathrm{X}$ and $\mathrm{P} 2 \mathrm{Y}$ receptor subtypes in models of neuropathic, acute and inflammatory pain. Br J Pharmacol. 2010;159(2010):1106-17.

[63] Surprenant A, Rassendren F, Kawashima E, North RA, Buell G. The cytolytic P2Z receptor for extracellular ATP identified as a P2X receptor (P2X7). Science.

1996;272(1996):735-8.

[64] Chessell IP, Hatcher JP, Bountra C, Michel AD, Hughes JP, Green P, et al. Disruption of the $\mathrm{P} 2 \mathrm{X} 7$ purinoceptor gene abolishes chronic inflammatory and neuropathic pain. Pain. 2005;114(2005):386-96.

[65] Honore P, Donnelly-Roberts D, Namovic MT, Hsieh G, Zhu CZ, Mikusa JP, et al. A740003 [N-(1-\{[(cyanoimino)(5-quinolinylamino) methyl]amino $\}$-2,2-dimethylpropyl)-2(3,4-dimethoxyphenyl)acetamide], a novel and selective P2X7 receptor antagonist, dosedependently reduces neuropathic pain in the rat. J Pharmacol Exp Ther. 2006;319(2006):1376-85.

[66] McGaraughty S, Chu KL, Namovic MT, Donnelly-Roberts DL, Harris RR, Zhang XF, et al. P2X7-related modulation of pathological nociception in rats. Neuroscience. 2007;146(2007):1817-28.

[67] Itoh K, Chiang CY, Li Z, Lee JC, Dostrovsky JO, Sessle BJ. Central sensitization of nociceptive neurons in rat medullary dorsal horn involves purinergic $\mathrm{P} 2 \mathrm{X} 7$ receptors.

Neuroscience. 2011;192(2011):721-31. 
[68] Burstein R, Yarnitsky D, Goor-Aryeh I, Ransil BJ, Bajwa ZH. An association between migraine and cutaneous allodynia. Ann Neurol. 2000;47(2000):614-24.

[69] Ito G, Suekawa Y, Watanabe M, Takahashi K, Inubushi T, Murasaki K, et al. P2X7 receptor in the trigeminal sensory nuclear complex contributes to tactile allodynia/hyperalgesia following trigeminal nerve injury. Eur J Pain. 2013;17(2013):185-99.

[70] Goloncser F, Sperlagh B. Effect of genetic deletion and pharmacological antagonism of P2X7 receptors in a mouse animal model of migraine. J Headache Pain. 2014;15(2014):24.

[71] Jiang LH, Mackenzie AB, North RA, Surprenant A. Brilliant blue G selectively blocks ATP-gated rat P2X(7) receptors. Mol Pharmacol. 2000;58(2000):82-8.

[72] He WJ, Cui J, Du L, Zhao YD, Burnstock G, Zhou HD, Ruan HZ. Spinal P2X(7) receptor mediates microglia activation-induced neuropathic pain in the sciatic nerve injury rat model. Behav Brain Res. 2012;226(2012):163-70.

[73] Burnell JM, Kirby WM. Effectiveness of a new compound, benemid, in elevating serum penicillin concentrations. J Clin Invest. 1951;30(1951):697-700.

[74] Gutman AB, Yu TF. Benemid (p-di-n-propylsulfamyl)-benzoic acid) as uricosuric agent in chronic gouty arthritis. Trans Assoc Am Physicians. 1951;64(1951):279-88.

[75] van Aubel RA, Smeets PH, Peters JG, Bindels RJ, Russel FG. The MRP4/ABCC4 gene encodes a novel apical organic anion transporter in human kidney proximal tubules: putative efflux pump for urinary cAMP and cGMP. J Am Soc Nephrol. 2002;13(2002):595603.

[76] Reid G, Wielinga P, Zelcer N, van der Heijden I, Kuil A, de Haas M, et al. The human multidrug resistance protein MRP4 functions as a prostaglandin efflux transporter and is inhibited by nonsteroidal antiinflammatory drugs. Proc Natl Acad Sci U S A.

2003;100(2003):9244-9.

[77] McClenaghan C, Zeng F, Verkuyl JM. TRPA1 agonist activity of probenecid desensitizes channel responses: consequences for screening. Assay Drug Dev Technol. 2012;10(2012):533-41.

[78] Bang S, Kim KY, Yoo S, Lee SH, Hwang SW. Transient receptor potential V2 expressed in sensory neurons is activated by probenecid. Neurosci Lett. 2007;425(2007):1205.

[79] Shimosato G, Amaya F, Ueda M, Tanaka Y, Decosterd I, Tanaka M. Peripheral inflammation induces up-regulation of TRPV2 expression in rat DRG. Pain. 2005;119(2005):225-32.

[80] Bandell M, Story GM, Hwang SW, Viswanath V, Eid SR, Petrus MJ, et al. Noxious cold ion channel TRPA1 is activated by pungent compounds and bradykinin. Neuron. 2004;41(2004):849-57. 
[81] Mercado J, Gordon-Shaag A, Zagotta WN, Gordon SE. Ca2+-dependent desensitization of TRPV2 channels is mediated by hydrolysis of phosphatidylinositol 4,5bisphosphate. J Neurosci. 2010;30(2010):13338-47.

[82] Silverman W, Locovei S, Dahl G. Probenecid, a gout remedy, inhibits pannexin 1 channels. Am J Physiol Cell Physiol. 2008;295(2008):C761-7.

[83] Silverman WR, de Rivero Vaccari JP, Locovei S, Qiu F, Carlsson SK, Scemes E, et al. The pannexin 1 channel activates the inflammasome in neurons and astrocytes. J Biol Chem. 2009;284(2009):18143-51.

[84] Pelegrin P, Surprenant A. Pannexin-1 mediates large pore formation and interleukin1 beta release by the ATP-gated P2X7 receptor. EMBO J. 2006;25(2006):5071-82.

[85] Pelegrin P, Surprenant A. The P2X(7) receptor-pannexin connection to dye uptake and IL-1beta release. Purinergic Signal. 2009;5(2009):129-37.

[86] Bravo D, Maturana CJ, Pelissier T, Hernández A, Constandil L. Interactions of pannexin 1 with NMDA and P2X7 receptors in central nervous system pathologies: Possible role on chronic pain. Pharmacol Res. 2015;101(2015):86-93.

[87] Samsam M, Covenas R, Ahangari R, Yajeya J, Narvaez JA, Tramu G. Alterations in neurokinin A-, substance P- and calcitonin gene-related peptide immunoreactivities in the caudal trigeminal nucleus of the rat following electrical stimulation of the trigeminal ganglion. Neurosci Lett. 1999;261(1999):179-82.

[88] Paxinos G, Watson C. The rat brain in stereotaxic coordinates. 6th ed. Amsterdam ; Boston: Elsevier, 2007.

[89] Knyihar-Csillik E, Tajti J, Samsam M, Sary G, Slezak S, Vecsei L. Effect of a serotonin agonist (sumatriptan) on the peptidergic innervation of the rat cerebral dura mater and on the expression of c-fos in the caudal trigeminal nucleus in an experimental migraine model. J Neurosci Res. 1997;48(1997):449-64.

[90] Knyihár-Csillik E, Toldi J, Krisztin-Péva B, Chadaide Z, Németh H, Fenyo R, Vécsei L. Prevention of electrical stimulation-induced increase of $c$-fos immunoreaction in the caudal trigeminal nucleus by kynurenine combined with probenecid. Neurosci Lett. 2007;418(2007):122-6.

[91] Hermann DM, Luppi PH, Peyron C, Hinckel P, Jouvet M. Afferent projections to the rat nuclei raphe magnus, raphe pallidus and reticularis gigantocellularis pars alpha demonstrated by iontophoretic application of choleratoxin (subunit b). J Chem Neuroanat. 1997;13(1997):1-21.

[92] Sugiyo S, Takemura M, Dubner R, Ren K. Trigeminal transition zone/rostral ventromedial medulla connections and facilitation of orofacial hyperalgesia after masseter inflammation in rats. J Comp Neurol. 2005;493(2005):510-23. 
[93] Hoskin KL, Bulmer DC, Lasalandra M, Jonkman A, Goadsby PJ. Fos expression in the midbrain periaqueductal grey after trigeminovascular stimulation. J Anat.

2001;198(2001):29-35.

[94] Marchand JE, Hagino N. Afferents to the periaqueductal gray in the rat. A horseradish peroxidase study. Neuroscience. 1983;9(1983):95-106.

[95] Teixeira JM, Oliveira MC, Nociti FH, Jr., Clemente-Napimoga JT, Pelegrini-da-Silva A, Parada CA, Tambeli CH. Involvement of temporomandibular joint P2X3 and P2X2/3 receptors in carrageenan-induced inflammatory hyperalgesia in rats. Eur J Pharmacol. 2010;645(2010):79-85.

[96] D'Amico M, Samengo I, Navarra P, Taglialatela M, Martire M. AMPA- and P2X7receptor-mediated facilitation of $[3 \mathrm{H}] \mathrm{D}$-aspartate release from nerve terminals isolated from the rat caudal brainstem. Neurochem Int. 2010;57(2010):623-8.

[97] Matsuka Y, Neubert JK, Maidment NT, Spigelman I. Concurrent release of ATP and substance $\mathrm{P}$ within guinea pig trigeminal ganglia in vivo. Brain Res. 2001;915(2001):248-55.

[98] Knyihar-Csillik E, Tajti J, Samsam M, Sary G, Buzas P, Vecsei L. Depletion of calcitonin gene-related peptide from the caudal trigeminal nucleus of the rat after electrical stimulation of the Gasserian ganglion. Exp Brain Res. 1998;118(1998):111-4.

[99] Borsani E, Albertini R, Labanca M, Lonati C, Rezzani R, Rodella LF. Peripheral purinergic receptor modulation influences the trigeminal ganglia nitroxidergic system in an experimental murine model of inflammatory orofacial pain. J Neurosci Res.

2010;88(2010):2715-26.

[100] Greco R, Tassorelli C, Sandrini G, Di Bella P, Buscone S, Nappi G. Role of calcitonin gene-related peptide and substance $\mathrm{P}$ in different models of pain. Cephalalgia.

2008;28(2008):114-26.

[101] Jo S, Bean BP. Inhibition of neuronal voltage-gated sodium channels by brilliant blue G. Mol Pharmacol. 2011;80(2011):247-57.

[102] Diaz-Hernandez JI, Gomez-Villafuertes R, Leon-Otegui M, Hontecillas-Prieto L, Del Puerto A, Trejo JL, et al. In vivo P2X7 inhibition reduces amyloid plaques in Alzheimer's disease through GSK3beta and secretases. Neurobiol Aging. 2012;33(2012):1816-28.

[103] Hunt SP, Pini A, Evan G. Induction of c-fos-like protein in spinal cord neurons following sensory stimulation. Nature. 1987;328(1987):632-4.

[104] Pardutz A, Krizbai I, Multon S, Vecsei L, Schoenen J. Systemic nitroglycerin increases nNOS levels in rat trigeminal nucleus caudalis. Neuroreport. 2000;11(2000):3071-5.

[105] Vamos E, Pardutz A, Fejes A, Tajti J, Toldi J, Vecsei L. Modulatory effects of probenecid on the nitroglycerin-induced changes in the rat caudal trigeminal nucleus. Eur $\mathbf{J}$ Pharmacol. 2009;621(2009):33-7. 
[106] Moriyama T, Higashi T, Togashi K, Iida T, Segi E, Sugimoto Y, et al. Sensitization of TRPV1 by EP1 and IP reveals peripheral nociceptive mechanism of prostaglandins. Mol Pain. 2005;1(2005):3.

[107] Wang Y. The functional regulation of TRPV1 and its role in pain sensitization. Neurochem Res. 2008;33(2008):2008-12.

[108] Zhang RX, Mi ZP, Qiao JT. Changes of spinal substance P, calcitonin gene-related peptide, somatostatin, Met-enkephalin and neurotensin in rats in response to formalin-induced pain. Regul Pept. 1994;51(1994):25-32.

[109] Jenkins DW, Langmead CJ, Parsons AA, Strijbos PJ. Regulation of calcitonin generelated peptide release from rat trigeminal nucleus caudalis slices in vitro. Neurosci Lett. 2004;366(2004):241-4.

[110] Simmons DL, Botting RM, Hla T. Cyclooxygenase isozymes: the biology of prostaglandin synthesis and inhibition. Pharmacol Rev. 2004;56(2004):387-437.

[111] Neeb L, Hellen P, Boehnke C, Hoffmann J, Schuh-Hofer S, Dirnagl U, Reuter U. IL1 beta stimulates COX-2 dependent PGE(2) synthesis and CGRP release in rat trigeminal ganglia cells. PLoS One. 2011;6(2011):e17360.

[112] Gao Y, Duan YZ. Increased COX2 in the trigeminal nucleus caudalis is involved in orofacial pain induced by experimental tooth movement. Anat Rec (Hoboken).

2010;293(2010):485-91.

[113] Myren M, Baun M, Ploug KB, Jansen-Olesen I, Olesen J, Gupta S. Functional and molecular characterization of prostaglandin E2 dilatory receptors in the rat craniovascular system in relevance to migraine. Cephalalgia. 2010;30(2010):1110-22.

[114] Patwardhan AM, Vela J, Farugia J, Vela K, Hargreaves KM. Trigeminal nociceptors express prostaglandin receptors. J Dent Res. 2008;87(2008):262-6.

[115] Price TJ, Patwardhan A, Akopian AN, Hargreaves KM, Flores CM. Modulation of trigeminal sensory neuron activity by the dual cannabinoid-vanilloid agonists anandamide, $\mathrm{N}$ arachidonoyl-dopamine and arachidonyl-2-chloroethylamide. Br J Pharmacol.

2004;141(2004):1118-30.

[116] Jenkins DW, Feniuk W, Humphrey PP. Characterization of the prostanoid receptor types involved in mediating calcitonin gene-related peptide release from cultured rat trigeminal neurones. Br J Pharmacol. 2001;134(2001):1296-302.

[117] Matsumura K, Watanabe Y, Imai-Matsumura K, Connolly M, Koyama Y, Onoe H. Mapping of prostaglandin E2 binding sites in rat brain using quantitative autoradiography. Brain Res. 1992;581(1992):292-8.

[118] Antonova M, Wienecke T, Olesen J, Ashina M. Prostaglandin E(2) induces immediate migraine-like attack in migraine patients without aura. Cephalalgia. 2012;32(2012):822-33. 
[119] Huang D, Li S, Dhaka A, Story GM, Cao YQ. Expression of the transient receptor potential channels TRPV1, TRPA1 and TRPM8 in mouse trigeminal primary afferent neurons innervating the dura. Mol Pain. 2012;8(2012):66.

[120] Lee SM, Cho YS, Kim TH, Jin MU, Ahn DK, Noguchi K, Bae YC. An ultrastructural evidence for the expression of transient receptor potential ankyrin 1 (TRPA1) in astrocytes in the rat trigeminal caudal nucleus. J Chem Neuroanat. 2012;45(2012):45-9.

[121] Akopian AN, Ruparel NB, Jeske NA, Patwardhan A, Hargreaves KM. Role of ionotropic cannabinoid receptors in peripheral antinociception and antihyperalgesia. Trends Pharmacol Sci. 2009;30(2009):79-84.

[122] Materazzi S, Benemei S, Fusi C, Gualdani R, De Siena G, Vastani N, et al. Parthenolide inhibits nociception and neurogenic vasodilatation in the trigeminovascular system by targeting the TRPA1 channel. Pain. 2013;154(2013):2750-8.

[123] Bruzzone R, Hormuzdi SG, Barbe MT, Herb A, Monyer H. Pannexins, a family of gap junction proteins expressed in brain. Proc Natl Acad Sci U S A. 2003;100(2003):13644-9.

[124] Takeda M, Tanimoto T, Kadoi J, Nasu M, Takahashi M, Kitagawa J, Matsumoto S. Enhanced excitability of nociceptive trigeminal ganglion neurons by satellite glial cytokine following peripheral inflammation. Pain. 2007;129(2007):155-66.

[125] Guo W, Wang H, Watanabe M, Shimizu K, Zou S, LaGraize SC, et al. Glial-cytokineneuronal interactions underlying the mechanisms of persistent pain. J Neurosci.

2007;27(2007):6006-18.

[126] Takahashi K, Watanabe M, Suekawa Y, Ito G, Inubushi T, Hirose N, et al. IL-1beta in the trigeminal subnucleus caudalis contributes to extra-territorial allodynia/hyperalgesia following a trigeminal nerve injury. Eur J Pain. 2011;15(2011):467 e1-14.

[127] Leklem JE. Quantitative aspects of tryptophan metabolism in humans and other species: a review. Am J Clin Nutr. 1971;24(1971):659-72.

[128] Vécsei L, Miller J, MacGarvey U, Beal MF. Kynurenine and probenecid inhibit pentylenetetrazol- and NMDLA-induced seizures and increase kynurenic acid concentrations in the brain. Brain Res Bull. 1992;28(1992):233-8.

[129] Perwitasari O, Yan X, Johnson S, White C, Brooks P, Tompkins SM, Tripp RA. Targeting organic anion transporter 3 with probenecid as a novel anti-influenza a virus strategy. Antimicrob Agents Chemother. 2013;57(2013):475-83.

[130] Chiba S, Ikawa T, Takeshita H, Ichiba K, Sagi M, Mukai T, Anzai N. Interactions of human organic anion transporter 1 (hOAT1) with substances associated with forensic toxicology. Leg Med (Tokyo). 2011;13(2011):180-5.

[131] Colín-González AL, Santamaría A. Probenecid: an emerging tool for neuroprotection. CNS Neurol Disord Drug Targets. 2013;12(2013):1050-65. 
[132] van Wijk M, Sebens JB, Korf J. Probenecid-induced increase of 5-hydroxytryptamine synthesis in rat brain, as measured by formation of 5-hydroxytryptophan.

Psychopharmacology (Berl). 1979;60(1979):229-35.

[133] Näsström J, Karlsson U, Post C. Antinociceptive actions of different classes of excitatory amino acid receptor antagonists in mice. Eur J Pharmacol. 1992;212(1992):21-9.

[134] Mecs L, Tuboly G, Nagy E, Benedek G, Horvath G. The peripheral antinociceptive effects of endomorphin-1 and kynurenic acid in the rat inflamed joint model. Anesth Analg. 2009;109(2009):1297-304.

[135] Vecsei L, Szalardy L, Fulop F, Toldi J. Kynurenines in the CNS: recent advances and new questions. Nature Reviews: Drug Discovery. 2013;12(2013):64-82.

[136] Vámos E, Párdutz A, Varga H, Bohár Z, Tajti J, Fülöp F, et al. 1-Kynurenine combined with probenecid and the novel synthetic kynurenic acid derivative attenuate nitroglycerininduced nNOS in the rat caudal trigeminal nucleus. Neuropharmacology. 2009;57(2009):4259.

[137] Vámos E, Fejes A, Koch J, Tajti J, Fülöp F, Toldi J, et al. Kynurenate derivative attenuates the nitroglycerin-induced CamKII $\alpha$ and CGRP expression changes. Headache. 2010;50(2010):834-43.

[138] Kessler M, Terramani T, Lynch G, Baudry M. A glycine site associated with Nmethyl-D-aspartic acid receptors: characterization and identification of a new class of antagonists. J Neurochem. 1989;52(1989):1319-28.

[139] Wang J, Simonavicius N, Wu X, Swaminath G, Reagan J, Tian H, Ling L. Kynurenic acid as a ligand for orphan $\mathrm{G}$ protein-coupled receptor GPR35. J Biol Chem.

2006;281(2006):22021-8. 
I. 


\title{
Diverse effects of Brilliant Blue G administration in models of trigeminal activation in the rat
}

\author{
Zsuzsanna Bohár $^{1}$ • Gábor Nagy-Grócz ${ }^{2}$ Annamária Fejes-Szabó ${ }^{2}$ • \\ Lilla Tar $^{3}$ • Anna M. László ${ }^{4}$ - Alexandra Büki ${ }^{5}$ Nikolett Szabadi ${ }^{2}$. \\ Veronika Vraukó $^{2} \cdot$ László Vécsei $^{1,2}$ - Árpád Párdutz ${ }^{2}$
}

Received: 7 June 2015/ Accepted: 14 August 2015

(C) Springer-Verlag Wien 2015

\begin{abstract}
Activation of the trigeminal system plays an important role in the pathomechanism of headaches. A better understanding of trigeminal pain processing is expected to provide information helping to unravel the background of these diseases. ATP, a key modulator of nociceptive processing, acts on ligand-gated $\mathrm{P} 2 \mathrm{X}$ receptors. Antagonists of the P2X7 receptors, such as Brilliant Blue G (BBG), have proved effective in several models of pain. We have investigated the effects of BBG after electrical stimulation of the trigeminal ganglion and in the orofacial formalin test in the rat. The right trigeminal ganglion of male rats was stimulated either with $5 \mathrm{~Hz}, 0.5 \mathrm{~mA}$ pulses for $5 \mathrm{~min}$ (mild procedure) or with $10 \mathrm{~Hz}, 0.5 \mathrm{~mA}$ pulses for $30 \mathrm{~min}$ (robust procedure), preceded by $50 \mathrm{mg} / \mathrm{kg}$ i.v. BBG. The animals were processed for c-Fos and calcitonin gene-related peptide (CGRP) immunohistochemistry. In the orofacial formalin test, $50 \mu \mathrm{L}$ of $1.5 \%$ formalin was injected into the right whisker pad of awake rats, following the pre-treatment with BBG. Behaviour was monitored for
\end{abstract}

László Vécsei

vecsei.laszlo@med.u-szeged.hu

1 MTA-SZTE Neuroscience Research Group, Semmelweis u. 6., Szeged 6725, Hungary

2 Department of Neurology, Faculty of Medicine, Albert SzentGyörgyi Clinical Center, University of Szeged, Semmelweis u. 6., Szeged 6725, Hungary

3 Department of Neurology, University of Ulm, Oberer Eselsberg 45, 89081 Ulm, Germany

4 Department of Medical Physics and Informatics, Faculty of Medicine, Faculty of Science and Informatics, University of Szeged, Korányi fasor 9, Szeged 6720, Hungary

5 Department of Physiology, Faculty of Medicine, University of Szeged, Dóm tér 10, Szeged 6720, Hungary
45 min, and c-Fos and CGRP immunohistochemistry was performed. BBG attenuated the increase in c-Fos-positive cells in the caudal trigeminal nucleus (TNC) after robust stimulation, but not after mild stimulation. No alterations in CGRP levels were found with either methodology. BBG did not mitigate either the behaviour or the increase in c-Fos-positive cells in the TNC during the orofacial formalin test. These results indicate that $\mathrm{P} 2 \mathrm{X} 7$ receptors may have a role in the modulation of nociception in the trigeminal system.

Keywords Trigeminal system $\cdot \mathrm{P} 2 \mathrm{X} 7$ receptor . Trigeminal ganglion stimulation $\cdot$ Formalin test

\section{Introduction}

Primary headaches are very common, but underdiagnosed and undertreated neurological conditions. Even following the correct diagnosis, the available therapeutic options often do not provide complete resolution of the pain, and recurrence of the headache after treatment is also a common complaint. Research is, therefore, currently focused on gaining an understanding of the causes of different headache disorders and developing new therapeutic options.

A common mechanism in primary headaches involves activation and sensitization of the trigeminal system, but the exact mechanism of these phenomena remains to be discovered (Tajti et al. 2011).

Recent results suggest that the purine molecule ATP has an important role in the regulation of nociceptive transmission (Burnstock 2013). Molecules targeting the specific receptors (Rs) for ATP (P2X and P2Y-Rs) have been proven to be effective in modulating different pain 
conditions, e.g., neuropathic and inflammatory pain (Ando et al. 2010). Among the ligand-gated P2X-Rs, the P2X7 receptor (P2X7-R) has been intensively studied in different pain states. The P2X7-R is a non-selective cation channel, unique among the $\mathrm{P} 2 \mathrm{X}$-Rs by virtue of its long $\mathrm{C}$ terminal domain, and its ability to open a pore permeable to molecules up to $900 \mathrm{Da}$ (Surprenant et al. 1996). Experiments with knock-out mice have revealed that the absence of the P2X7-R leads to the disappearance of mechanical and thermal hypersensitivity in models of neuropathic and inflammatory pain, whereas normal nociceptive processing is retained (Chessell et al. 2005). P2X7-R antagonists have been examined in similar models, and the results underline the importance of the P2X7-R in chronic pain conditions (Honore et al. 2006; McGaraughty et al. 2007). Results from an acute inflammatory pain model suggest that the P2X7-R may participate in the development of central sensitization (Itoh et al. 2011), a common feature in the trigeminal system during migraine attacks manifested by the presence of allodynia (Burstein et al. 2000).

However, little information is available as concerns the role of P2X7-Rs in the trigeminal system. In a model of orofacial pain, the chronic constriction injury model, inhibition of the P2X7-R led to a decrease in tactile allodynia through a p38 MAPK-dependent mechanism (Ito et al. 2013). Goloncser and Sperlagh recently reported that blockade of P2X7-Rs in mice by Brilliant Blue G (BBG) [a selective, non-competitive P2X7-R antagonist, with good blood-brain barrier permeability (Jiang et al. 2000)] reduced thermal hyperalgesia after systemic nitroglycerin administration (Goloncser and Sperlagh 2014). These results lend support to the theory that P2X7-Rs may play a crucial part in the development of headache disorders.

Calcitonin gene-related peptide (CGRP) has an essential role in trigeminal nociceptive processing. CGRP infusion causes migraine-like headache in migraineurs (Hansen et al. 2010; Lassen et al. 2002), and the levels of CGRP are higher in migraine patients than in healthy controls (Friberg et al. 1994).

Electrical stimulation of the trigeminal ganglion, an animal model of trigeminal activation, causes plasma protein extravasation (Markowitz et al. 1987), which can be attenuated by drugs effective in migraine therapy (Limmroth et al. 2001). The stimulation results in alterations in the dura mater (Knyihar-Csillik et al. 1995), in the trigeminal ganglion and in the caudal part of the spinal trigeminal nucleus (TNC) (Knyihar-Csillik et al. 1998). In previous investigations, various stimulation frequencies, intensities and durations were applied. Results related to peptide release and c-Fos expression in the TNC after the procedure suggest that both higher frequency and higher intensity lead to an increased stimulation of the trigeminal ganglion cells (Samsam et al. 1999; Takemura et al. 2000).
Diverse periods of stimulation caused different morphological alterations in the CGRP-immunoreactive nerve terminals of the dura mater, suggesting the release of CGRP after prolonged stimulation (Knyihar-Csillik et al. 1995). Based on these previous results, we decided to perform the electrical stimulation in two different setups: a mild and a robust one.

Formalin applied to the whisker pad of rats (another model of trigeminal activation) causes a biphasic behavioural effect, the first phase being caused by the direct activation of $\mathrm{A} \delta$ and $\mathrm{C}$ fibres, while the second phase reflects the process of inflammation (Porro and Cavazzuti 1993). Activation of the trigeminal system has been demonstrated by c-Fos immunohistochemistry to be present at the level of the TNC after the injection of formalin (Wang et al. 1994). Both phenomena are suitable for assessments of the influence of substances on inflammation.

The aim of the present study was to examine the effects of a P2X7-R antagonist, BBG, in the electrical stimulation and orofacial formalin models of acute trigeminal activation.

\section{Materials and methods}

\section{Ethical approval}

The procedures used in this study followed the guidelines of the 8th Edition of the Guide for the Care and Use of Laboratory Animals and the Use of Animals in Research of the International Association for the Study of Pain and the directive of the European Economic Community (86/609/ ECC). The experiments were approved by the Committee of Animal Research at the University of Szeged (I-74-12/ 2012) and the Scientific Ethics Committee for Animal Research of the Protection of Animals Advisory Board (XXIV/352/2012). Male Sprague-Dawley rats were housed under standard laboratory conditions, on a 12-h light-dark cycle, with tap water and rat chow available ad libitum. The suffering of the animals and the number of animals used were kept at a minimum.

\section{Mild stimulation procedure}

Twenty-four animals (250-300 g) were used. Half of the animals received an intravenous (i.v.) injection of $50 \mathrm{mg} /$ $\mathrm{kg} \mathrm{BBG}$, while the other half were injected with the vehicle of BBG, physiological saline. Two $h$ after the BBG or saline injection, the animals were deeply anesthetised with chloral hydrate $(400 \mathrm{mg} / \mathrm{kg}$ ) and the head was secured in a stereotaxic apparatus (Stoelting Co., Wood Dale, USA). A hole, approximately $3 \mathrm{~mm}$ in diameter, was drilled into the 
right side of the skull with a dental drill, and a concentric bipolar electrode (FHC Inc., Bowdoin, USA, CBBRE75) was lowered to the right trigeminal ganglion. Half of the animals from the saline-treated group (5SStim) and half of the animals from the BBG group (5BStim) were electrically stimulated for $5 \mathrm{~min}$ with $5 \mathrm{~Hz}, 0.5 \mathrm{~mA}, 0.5 \mathrm{~ms}$ delay twin pulses generated by an Electrostimulator ST3 (Medicor Hungary). The other animals from both groups were used as sham animals: the electrode was lowered to the right trigeminal ganglion for $5 \mathrm{~min}$, but no stimulation was performed (5SSham and 5BSham groups). After both procedures, the animals were returned to their home cages and maintained under deep anaesthesia covered by a warming blanket for $2 \mathrm{~h}$.

\section{Robust stimulation procedure}

Twenty-one animals (250-300 g) were used; the treatment and surgical procedures were identical to the previous ones, except that the stimulation parameters of $10 \mathrm{~Hz}, 0.5 \mathrm{~mA}$, $0.5 \mathrm{~ms}$ delay twin pulses were applied for $30 \mathrm{~min}$, and the animals were maintained under deep anaesthesia, covered by a warming blanket, in their home cages for $4 \mathrm{~h}$ from the beginning of the stimulation. In both the mild and the robust paradigm, the jaw of the animal was twitching during the electrical stimulation, indicating the correct placement of the electrode.

Overview of the robust stimulation groups:

$$
\begin{aligned}
& \text { Saline + 30-min sham: 30SSham }(n=6) . \\
& \text { Saline + 30-min stimulation: 30SStim }(n=5) . \\
& \text { BBG + 30-min sham: 30BSham }(n=4) . \\
& \text { BBG + 30-min stimulation: 30BStim }(n=6) .
\end{aligned}
$$

\section{Orofacial formalin test}

\section{Behavioural tests}

Rats $(n=52,200-240 \mathrm{~g})$ were injected i.v. either with $50 \mathrm{mg} / \mathrm{kg}$ BBG or with physiological saline. One hour and fifty minutes later, the animals were placed in a $30 \times 30 \times 30 \mathrm{~cm}$ box, with mirrored walls for the monitoring of behavioural activity. After $10 \mathrm{~min}$ of habituation, the animals were taken out of the box and under minimal restraint were injected subcutaneously with $50 \mu \mathrm{L}$ of either physiological saline (SSal and BSal groups) or $1.5 \%$ formalin (SForm and BForm groups) to the right whisker pad. After the injection, they were returned immediately to the box and their behaviour was monitored for 45 min under video surveillance. The injection of formalin causes a behavioural response, which consists in rubbing and scratching of the injected whisker pad with the ipsilateral fore- or hindpaw. The rate of this behaviour correlates with the pain sensation caused by formalin (Clavelou et al. 1989). The 45-min period was divided into $15 \times 3$-min blocks, and the total time spent rubbing the injected whisker pad, measured in seconds, was taken as the nociceptive score in the given block. The normal grooming activity of the saline-treated animals was measured as control. After the monitoring period, the animals were returned into their home cages and maintained under standard laboratory conditions until perfusion, which was performed under deep chloral hydrate anaesthesia $4 \mathrm{~h}$ after the whisker pad injections.

\section{Immunohistochemistry}

Animals were perfused with $0.1 \mathrm{M}$ phosphate-buffered saline (PBS), followed by $4 \%$ paraformaldehyde in $0.1 \mathrm{M}$ phosphate buffer. The whole brain and the upper cervical spinal cord were removed and postfixed overnight in the same fixative. The correct placement of the electrode was checked during autopsy, the electrode was in the ganglion in all cases. Cryoprotection was performed, using gradient sucrose solutions up to $30 \%$. Sections from the TNC were prepared from the block, ranging from $1 \mathrm{~mm}$ rostral to $4 \mathrm{~mm}$ caudal from the obex, and the ventral part of the control (left) side of the blocks was marked by a small incision to enable side discrimination on the sections. $30 \mu \mathrm{m}$ thick transverse sections were cut and serially collected in 18 wells containing PBS with $0.1 \%$ sodium azide, the overall distance therefore being $540 \mu \mathrm{m}$ between consecutive sections. Free-floating sections were immersed in $0.3 \% \mathrm{H}_{2} \mathrm{O}_{2}$ in PBS to block endogenous peroxidase activity. After several washes in PBS containing $1 \%$ Triton X-100 (PBS-T), they were incubated for $1 \mathrm{~h}$ in PBS-T containing $10 \%$ normal goat serum. The sections were then incubated overnight at room temperature in the primary antibody for c-Fos (1:2000, Santa Cruz Biotechnology, sc-52) or CGRP (1:20,000, Sigma-Aldrich C8198). The immunohistochemical reaction was visualised by using the Vectastain Elite avidin-biotin kit (PK6101; Vector Laboratories,) with 3,3'-diaminobenzidine as chromogen (Sigma-Aldrich) intensified with nickel ammonium sulphate (Scharlau Chemie). The specificity of the immune reactions was verified by omitting the primary antisera.

Sections were mounted onto glass slides, air-dried and coverslipped with DPX mounting medium (Scharlau Chemie). On the basis of anatomical observations, sections from the same rostro-caudal level were compared during the statistical evaluation.

An observer blinded to the treatment procedures used a Nikon Optiphot-2 light microscope under a $20 \times$ objective to count cells immunopositive for c-Fos. The whole area of the laminae I-II in each section of the TNC was evaluated and counted. 
Fig. 1 Summary of the results from the mild stimulation paradigm regarding c-Fos immunostaining. Representative photos from the right sides of the four treatment groups after c-Fos immunohistochemistry, taken at $16.05 \mathrm{~mm}$ caudally from bregma: a 5SSham, b 5SStim, c 5BSham, d 5BStim. Scale bar $200 \mu \mathrm{m}$.

Diagram showing the number of c-Fos-immunoreactive cells across the different levels of the TNC after mild electrical stimulation of the trigeminal ganglion (group mean \pm SEM) (e). There was no significant difference between the control (left) sides (data not shown). For clarity, only the control side of the saline-treated stimulated group is presented. The hashmarks indicate significance in the comparison of the right sides of the 5SSham and 5SStim groups at different levels of the TNC $\left({ }^{\#} p<0.05 ;{ }^{\# \#} p<0.01\right.$;

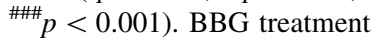
showed an attenuating tendency, though it proved to be significant merely at the level of $-13.89 \mathrm{~mm}(* * p<0.01$,

5SStim-5BStim) a

a

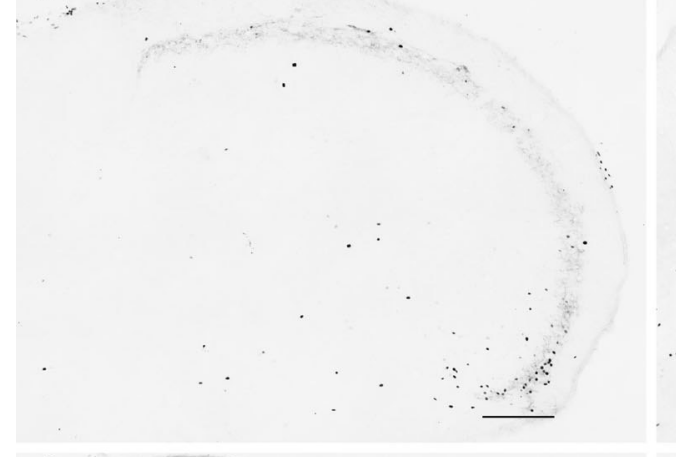

C

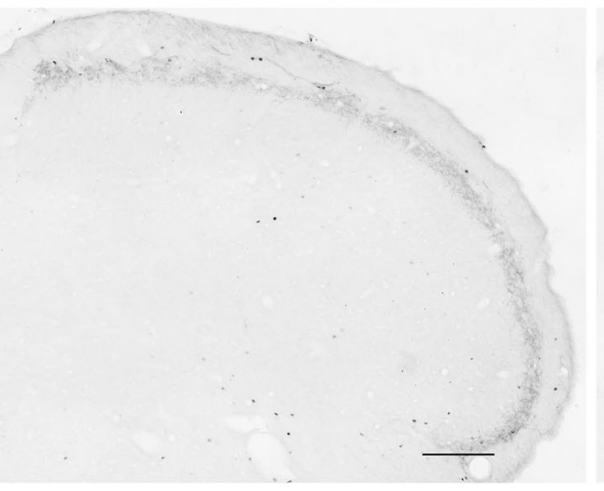

b

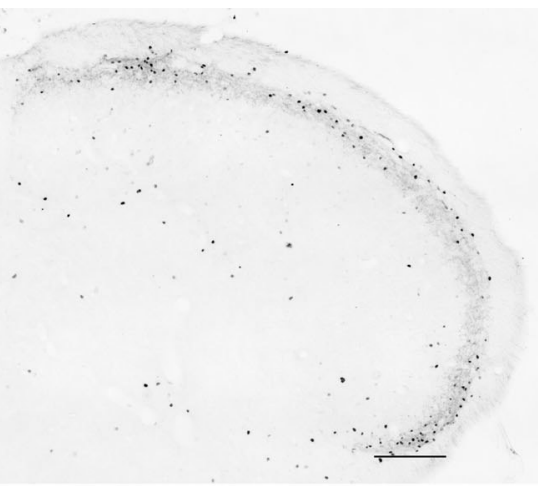

d

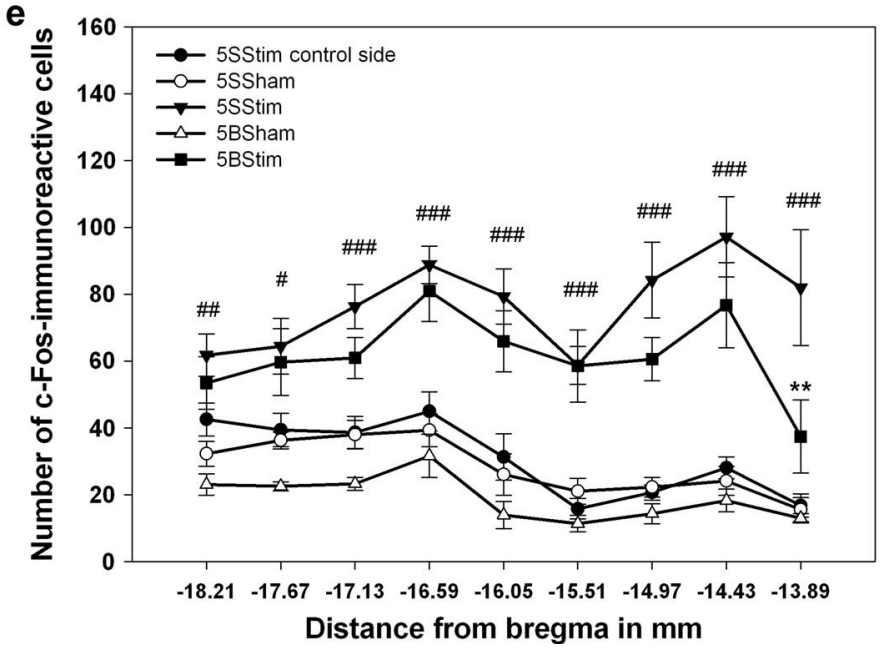

CGRP-stained sections were photographed with a Zeiss AxioCam MRc Rev.3 digital camera attached to a Zeiss AxioImager M2 microscope. Digital images were taken with a $20 \times$ objective in TNC laminae I-II, and the area covered by CGRP-immunoreactive fibres was measured through the use of ImageProPlus 6.2 software (Media Cybernetics Inc.) by an observer blinded to the treatment procedures.

\section{Statistical analysis}

The cell count results were aligned according to the rostrocaudal location of the section as mentioned above. Data from different levels of the TNC were handled separately, and analysed by two-way repeated measures ANOVA. The group was used as the between-subject factor and the levels $(-13.89,-14.43,-14.97,-15.51,-16.05,-16.59$, $-17.13,-17.67,-18.21 \mathrm{~mm}$ from bregma) as the withinsubject factor for the analysis.

When Mauchly's test of sphericity proved to be significant, the Greenhouse-Geisser correction was performed. Pairwise comparisons of group means were performed on the basis of estimated marginal means with Sidak adjustment for multiple comparisons.

The sums of the areas covered by CGRP-immunoreactive fibres were compared between groups according to the 


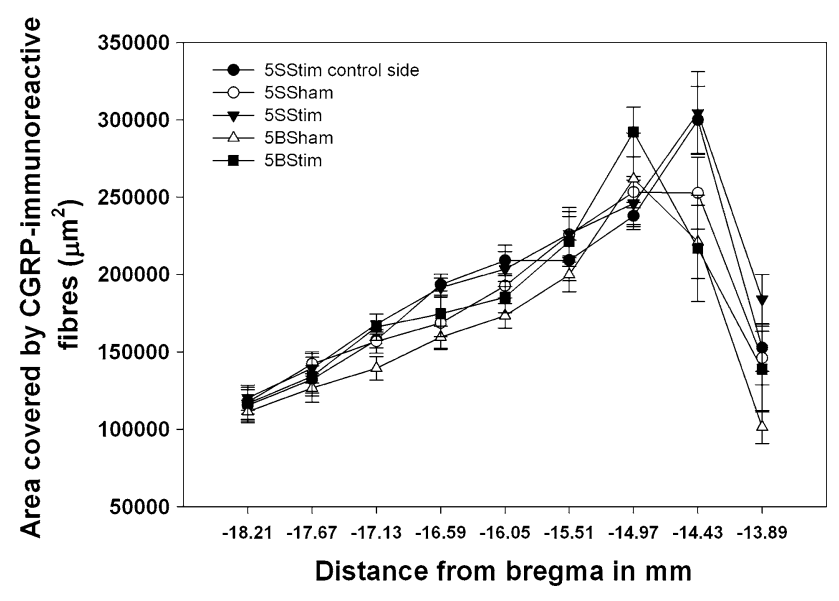

Fig. 2 Diagram showing the area covered by CGRP-immunoreactive fibres at the different levels of the TNC in the different treatment groups after mild electrical stimulation of the trigeminal ganglion (group mean $\pm \mathrm{SEM}$ ). There was no significant difference between either the control or the stimulated sides or the different groups. For clarity, only the control side of the saline-treated stimulated group is presented

different levels, two-way repeated measures ANOVA being used as detailed above.

Nociceptive scores from the behavioural study were compared block by block through two-way repeated measures ANOVA. Groups were used as between-subject factor and blocks (1-15) as within-subject factor for the analysis. Other statistical parameters were identical to those mentioned above.

Statistical analyses were carried out with IBM SPSS Statistics, version 20 (IBM Corporation) software. All tests were two-sided, and $p<0.05$ was considered to be statistically significant. Graphs were prepared by using SigmaPlot 12.0 (Systat Software Inc.). Data are reported as mean \pm SEM.

\section{Results}

\section{Mild stimulation procedure}

As a significant interaction was found between the two investigated factors (levels and groups, $p<0.01$ ) for the number of c-Fos-immunoreactive cells in the mild stimulation paradigm, both effects could not be reported independently, whereas the group differences could be examined separately across different levels on the basis of the estimated marginal means for multiple comparisons.

Lowering of the electrode to the trigeminal ganglion for 5 min without stimulation (5SSham group) did not cause any significant change in the number of c-Fos-immunoreactive cells in the TNC relative to the control side. The comparisons of the cell numbers from the control (left) sides for each of the four treatment groups did not reveal any significant changes (data not shown), and therefore only the data for the stimulated (right) sides of the groups are presented in Fig. 1a-d. Electrical stimulation of the ganglion caused a significant increase in the number of c-Fos-immunoreactive (IR) cells along the whole extent of the examined region of the TNC (Fig. 1a, b, e). BBG exhibited a significant effect $(p<0.01)$ compared with the saline-treated stimulated animals only at the level of $-13.89 \mathrm{~mm}$ from bregma (Fig. 1b, c, e).

A significant interaction was found between the groups and levels, when the area values from the CGRP measurements were examined. However, the comparisons of the groups and stimulated-control sides at different levels did not reveal any significant alteration (Fig. 2).

\section{Robust stimulation procedure}

A significant interaction was not found between the investigated factors of levels and groups for the number of c-Fos IR cells in the robust stimulation paradigm. The levels had a significant effect $(p<0.001)$, and there was also a significant difference between the groups $(p<0.001)$. Pairwise comparisons revealed that there was no significant difference between the control sides of the four treatment groups (data not shown). Lowering of the electrode without stimulation did not cause significant changes in the number of c-Fos IR cells in either shamtreated group as compared with the control (Fig. 3e, 30SSham and 30BSham with the 30SStim control side). Robust stimulation caused a marked increase in the number of c-Fos IR cells in the saline-treated animals (Fig. 3a, b, e, 30SStim compared with 30SSham, $\left.{ }^{\#} p<0.001\right)$. BBG had a significant attenuating effect on this increase (Fig. 3b, d, e, 30SStim compared with 30BStim).

CGRP expression was not altered by stimulation or BBG administration in any of the animal groups (Fig. 4).

\section{Orofacial formalin test}

A significant interaction was found between time and groups $(p<0.05)$ during the analysis of the behavioural data from the orofacial formalin test. The pairwise comparison revealed that the nociceptive scores of the salineinjected groups (SSal and BSal) did not differ from each other at any time point (Fig. 5). The injection of formalin caused a significant increase in the nociceptive scores in blocks 1 and 5-7 (Fig. 5, SForm compared with SSal). After the injection of formalin into the whisker pad, the BBG-treated animals demonstrated significantly increased nociceptive scores in blocks 1,6 and 8 as compared with the control (Fig. $5,{ }^{\$} p<0.05$ ). The BBG-treated animals spent less time rubbing their formalin-injected side in both 
Fig. 3 Summary of the results from the robust stimulation paradigm regarding c-Fos immunostaining. Representative photos from the right sides of the four treatment groups after c-Fos immunohistochemistry, taken at $16.05 \mathrm{~mm}$ caudally from bregma: a 30SSham, b 30SStim, c 30BSham, d 30BStim. Scale bar $200 \mu \mathrm{m}$. Diagram showing the number of c-Fos-immunoreactive cells at different levels along the rostrocaudal axis in the TNC (group mean $\pm \mathrm{SEM}$ ) in the robust stimulation paradigm (e). There was no significant difference between the control sides (data not shown). For clarity, only the control side of the saline-treated stimulated group is presented. Electrical stimulation of the right trigeminal ganglion caused a significant increase in the number of c-Fos-positive cells as compared with the right side of the sham group at all levels examined (\#\#\#p 0.001

30SSham-30SStim). BBG treatment had a significant decreasing effect on the cell counts $(* * * p<0.00130$ SStim30BStim)
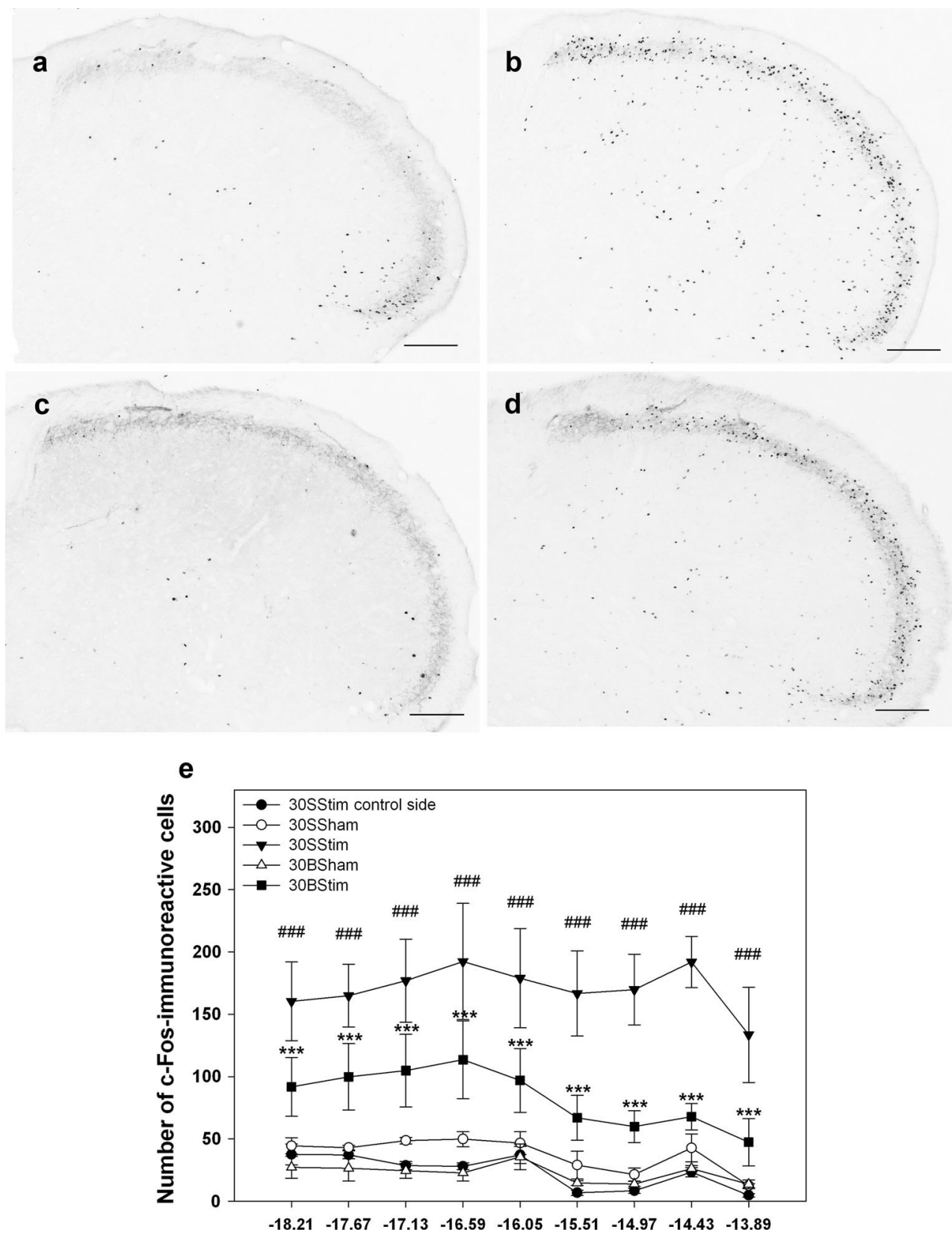

blocks 1 and 5-7, but this difference was not significant compared with the SForm group. In block 8, BBG-treated animals spent significantly more time rubbing their whisker pad as did the animals in the formalin group (Fig. 5). In our experiments, the second phase of the formalin test subsided more quickly as expected in the SForm group, while it was more prolonged in the BForm group, and this difference may account for the significant effect of BBG in block 8 .

A significant interaction was observed between the levels and groups $(p<0.001)$ for the number of c-Fos IR cells in the orofacial formalin test. There was no significant difference when either the control (left) sides or the control and saline-injected sides were compared at any level (Fig. 6e). The injection of formalin increased the number of c-Fos-IR cells significantly at the levels -16.59 to $-15.51 \mathrm{~mm}$, mainly in the central part of the TNC as compared with the saline-injected side in the SSal group. In the BForm group, a similar pattern was observed, except that the difference involved one additional level $(-14.97 \mathrm{~mm}$, Fig. 6e). There was no significant difference between the SForm and BForm groups at any level (Fig. 6b, d).

No group difference was found in any of the measured parameters as regards the CGRP immunoreactivity (Fig. 7). 


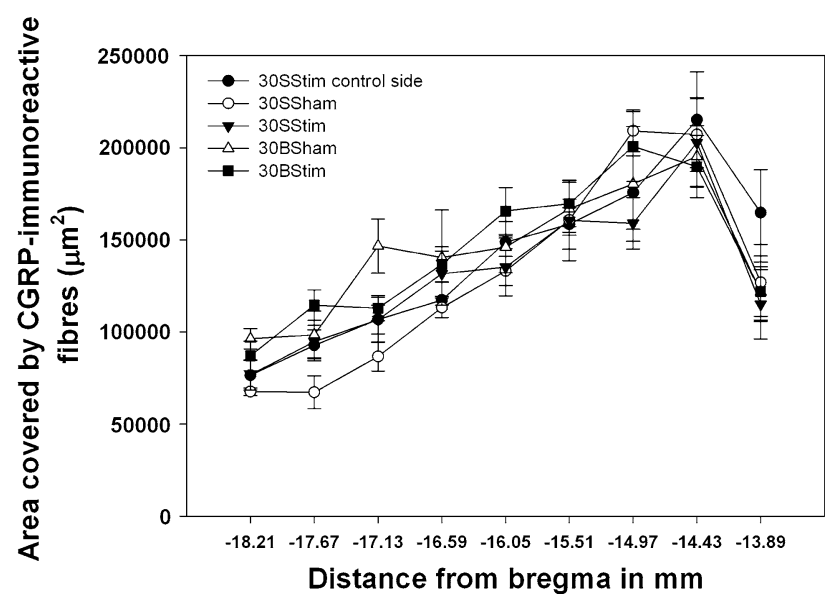

Fig. 4 Diagram showing the area covered by CGRP-immunoreactive fibres at the different levels of the TNC in the different treatment groups after robust electrical stimulation of the trigeminal ganglion (group mean \pm SEM). There was no significant difference between either the control or the stimulated sides or the different groups. For clarity, only the control side of the saline-treated stimulated group is presented

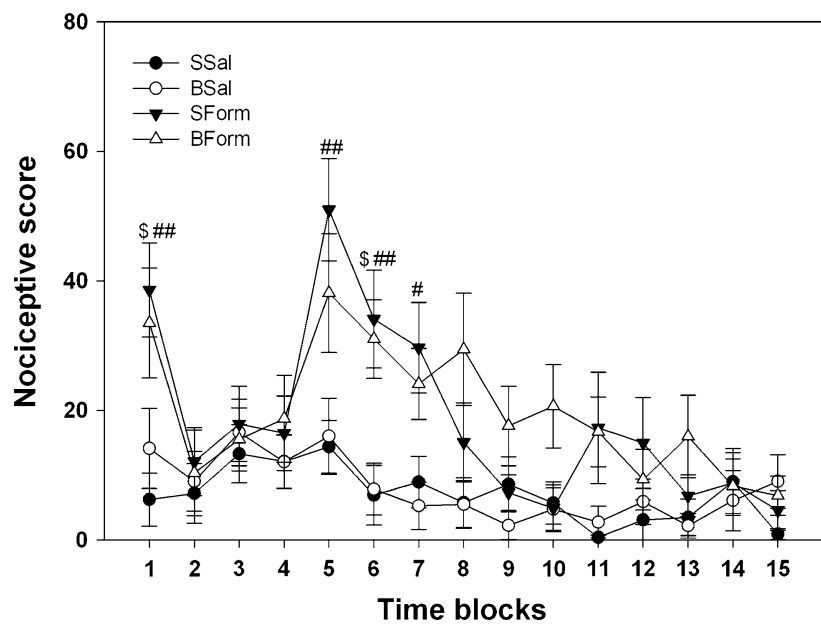

Fig. 5 Diagram showing the mean nociceptive scores in different time blocks from the four treatment groups (group mean \pm SEM, $n=13$ ) in the orofacial formalin test. The injection of formalin increased the nociceptive score in blocks 1 and 5-7 as compared with the saline-injected animals $\left({ }^{\#} p<0.05,{ }^{\# \#} p<0.01\right)$. BBG treatment did not affect the nociceptive scores after saline injection, and did not modify the normal behaviour. The nociceptive scores of the BBG and saline-treated formalin-injected groups did not differ significantly in any block

\section{Discussion}

Numerous stimulation parameters and stimulation times have been applied in previous experiments, and we therefore decided to make use of two stimulation procedures, a short, mild stimulation and a longer, robust stimulation, in order to examine the possible effects of P2X7-R antagonism on trigeminal activation. As P2X7-R blockade was previously found to be effective in inflammatory conditions, we additionally examined the effects of BBG in a model of orofacial inflammation, the orofacial formalin test.

Mild, short electrical stimulation of the trigeminal ganglion has been reported to lead to activation of the trigeminal system (Takemura et al. 2000), and this was supported by our results. The activation may be direct, stemming from depolarization of the central terminals of the primary trigeminal afferents, or it may be indirect, resulting from the release of peripheral mediators (histamine, bradykinin, substance P or CGRP).

We observed a similar pattern in the robust stimulation paradigm, the number of c-Fos IR cells increasing profoundly, indicating the activation of the trigeminal system.

The main difference seen between the two paradigms was in the number of cells activated after stimulation. Following the robust stimulation procedure more c-Fos IR cells were found in the TNC, suggesting a higher degree of activation than in the mild stimulation procedure. This higher degree of activation may be attributed to the higher frequency applied in the robust paradigm, which can lead to the more rapid firing of the primary trigeminal neurons. An increased firing rate may cause increased levels of transmitter release at both central and peripheral terminals, resulting in a higher degree of activation at the TNC level (Samsam et al. 1999). It is also plausible that the longer stimulation interval leads to more primary trigeminal cells being activated in the TG, and hence in the TNC. We assume that in our experimental setting both the increased frequency and the increased stimulation interval contributed to the higher activation level in the TNC.

Pre-treatment with the P2X7-R antagonist BBG was effective only in the robust stimulation paradigm, resulting in a decrease of the activity, reflected by the c-Fos expression. P2X7-Rs can be found in the trigeminal system, both in the ganglion (Teixeira et al. 2010) and in the TNC (D'Amico et al. 2010), therefore BBG may modulate peripheral and central processes. BBG could modulate the nociceptive processing by interfering with the peripheral neurogenic inflammation, or by modulating non-synaptic communication within the ganglion (Matsuka et al. 2001). At the central level, BBG could affect P2X7-Rs on central presynaptic terminals and modulate glutamate release (D'Amico et al. 2010), and thereby influence nociceptive transmission. Presumably, after the robust stimulation, where the more pronounced peripheral activation and more severe inflammation also involve P2X7-Rs, the blocking effect of BBG manifests, while in the mild paradigm due to the minor changes the effect of BBG does not emerge.

Neither the mild nor the robust stimulation procedure caused alterations in the levels of CGRP. It was earlier found that electrical stimulation of the trigeminal ganglion 
Fig. 6 Summary of the results from the orofacial formalin test regarding $\mathrm{c}-\mathrm{Fos}$

immunostaining. Representative photos from the right (injected) sides of the four treatment groups after c-Fos

immunohistochemistry taken at $16.05 \mathrm{~mm}$ caudally from bregma: a SSal, b SForm, c BSal, d BForm. Scale bar $200 \mu \mathrm{m}$. Diagram showing the mean number of c-Fos immunoreactive cells along the rostro-caudal axis in the TNC in the orofacial formalin test (group mean \pm SEM) (e). Injection of saline did not cause any increase in the number of c-Fos-positive cells (SSal and $\mathrm{BSal}$ groups). The effect of formalin was visible only on the injected side; the control sides were similar to those in the saline-injected controls. For clarity, only the control side of the SForm animals is presented. Formalin caused an increase in c-Fos cell number on the ipsilateral side as compared with the saline-injected side of the controls (SForm compared with SSal), significant at the levels -16.59 to $-15.51 \mathrm{~mm}$

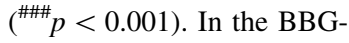
treated rats, formalin had a similar activity-increasing effect, significant at the levels

-16.59 to $-14.97 \mathrm{~mm}$

$\left({ }^{\$} p<0.05 ;{ }^{\$} p<0.01\right.$

$\$ \$ p<0.001)$. The groups

SForm and BForm did not differ significantly from each other

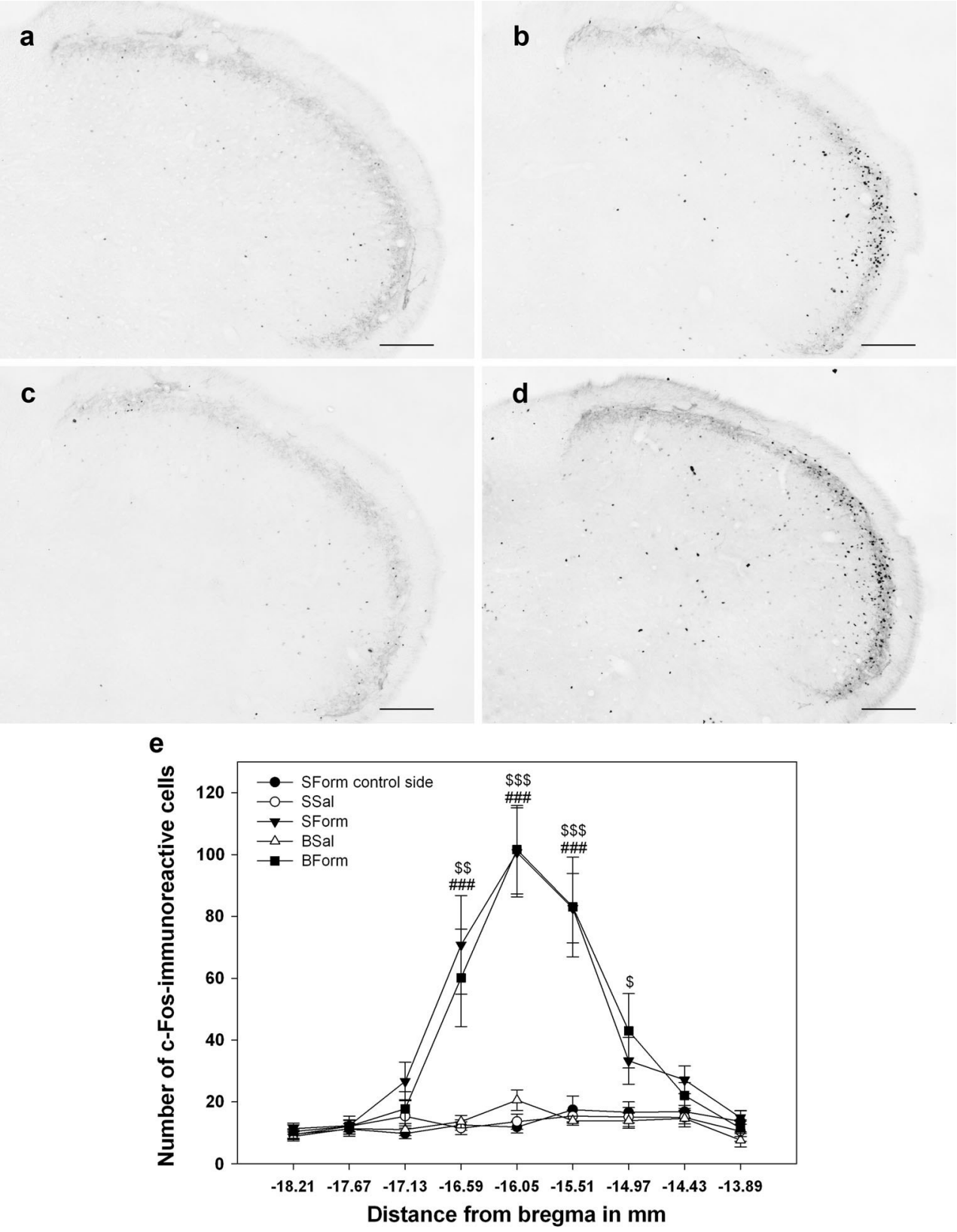

with parameters similar to our robust stimulation, led to the depletion of CGRP from the medial one-third of the central terminals of the trigeminal afferents (Knyihar-Csillik et al. 1998). However, those examinations were conducted immediately after stimulation of the trigeminal ganglion, whereas in our experiments a 2 or a 4 h survival time was included for better observability of the activity changes (cFos). These periods might be sufficient for the depleted CGRP to be resynthesised and for the changes in CGRP immunoreactivity seen immediately after stimulation to normalise. BBG treatment did not modify the levels of CGRP in either the sham or the stimulated group.

The injection of formalin into the whisker pad causes a biphasic behavioural effect (Clavelou et al. 1989), as also seen in our experiments. The first short and intense phase is thought to be caused by the immediate activation of the A $\delta$ and $\mathrm{C}$ fibres and may be referred as acute pain. The second phase is less intense, but prolonged, and probably caused by sensitization of the trigeminal system due to the inflammatory processes occurring at the periphery. BBG did not exhibit any effect in the first phase of the formalin response. When formalin was applied to the hind paw and was combined with a selective P2X7-R antagonist, A-438079, in previous work, protective effect was exerted only in the second phase of the formalin test (McGaraughty et al. 2007). Furthermore, BBG was earlier shown to be hyperalgesic in the modulation of acute nociception in the hot-plate test (Ando et al. 2010). These results suggest that 
Fig. 7 Summary of the results after CGRP immunostaining in Representative photos of CGRP immunohistochemistry on the right sides of the TNC from the four treatment groups taken at $14.97 \mathrm{~mm}$ caudally from bregma a 5SSham, b 5SStim, c 5BSham, d 5BStim. Scale bar $200 \mu \mathrm{m}$. Diagram showing the area covered by CGRPimmunoreactive fibres at the different levels of the TNC in the different treatment groups in the orofacial formalin test (group mean \pm SEM) (e). There was no significant difference between either the control or injected sides or the different groups. For clarity, only the control side of the saline-treated formalin-injected group is presented the orofacial formalin test.
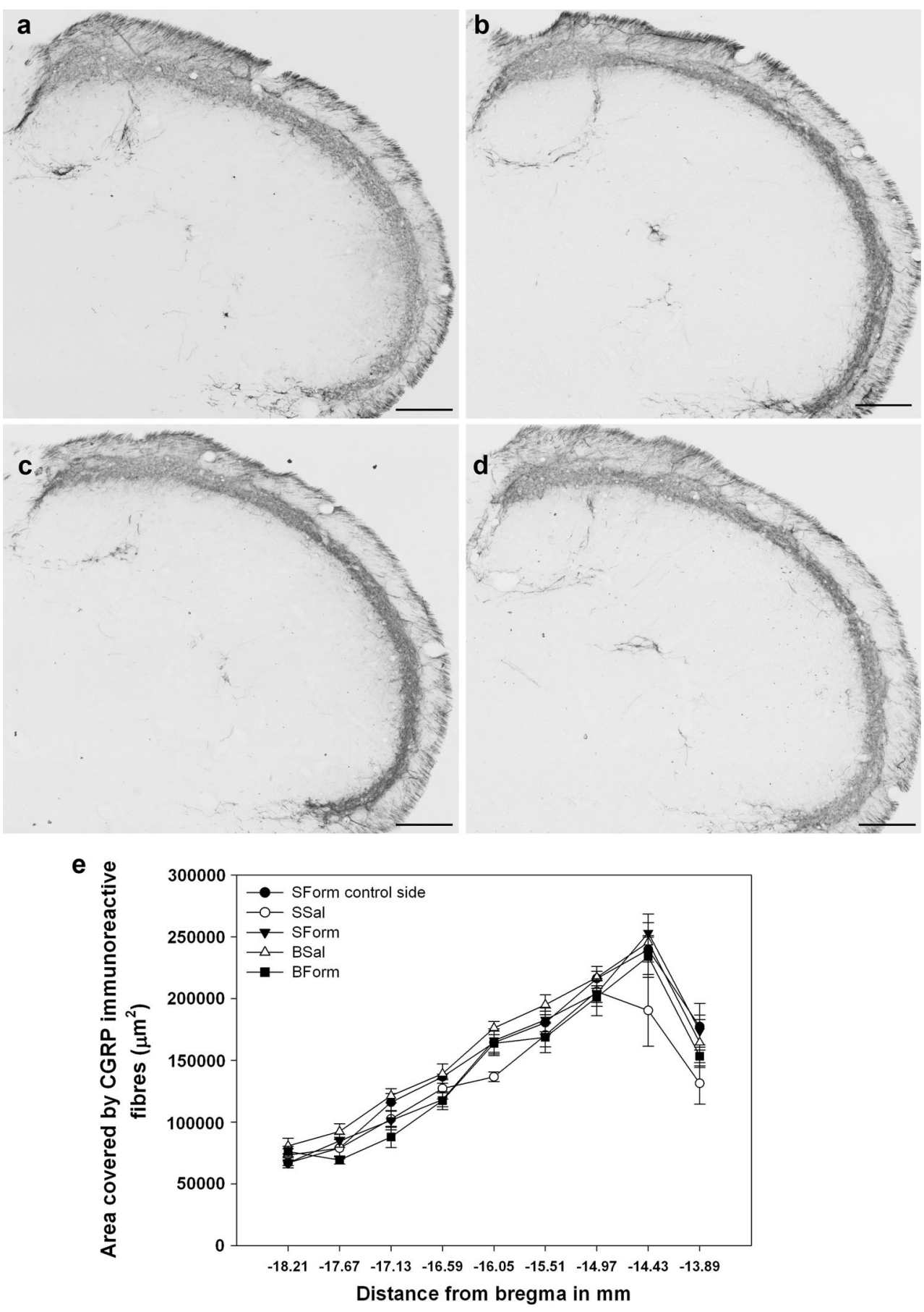

BBG and blockade of the P2X7-Rs may not be effective against acute nociception.

In the second phase of the formalin test, BBG did not demonstrate any obvious effect. At the beginning of the second phase (in blocks 5-7), the nociceptive scores revealed a decreasing tendency, while in the later blocks the opposite could be observed. Since another P2X7-R antagonist was effective when formalin was applied at the hind paws, our results suggest that the role of the P2X7-Rs in the sensory system is not uniform.
Four $\mathrm{h}$ after formalin injection, c-Fos immunohistochemistry revealed that the TNC displays clear activation. The pattern of activation corresponds to the somatotopic projection pattern of the injected area. BBG had no effect on the activation of the trigeminal system after formalin. Our results in the orofacial formalin test are somewhat surprising, considering that other antagonists of the P2X7Rs (Borsani et al. 2010; Honore et al. 2006; McGaraughty et al. 2007) and even BBG (Ando et al. 2010) have proven effective in numerous inflammatory models. However, 
none of these experiments related to the trigeminal system, and our results are the first regarding the effects of blockade of the P2X7-Rs by BBG in this area after inflammation caused by formalin.

The levels of CGRP were not altered $4 \mathrm{~h}$ after formalin injection, and following treatment with BBG. Alterations in CGRP usually occur immediately during or after the applied stimulus and cease within a matter of hours (Buzzi et al. 1991; Greco et al. 2008), and our results agree with this. However, in the nitroglycerin model, changes in CGRP immunoreactivity were seen $4 \mathrm{~h}$ after nitroglycerin administration, suggesting that the alterations in CGRP levels can be long-term. The effect of P2X7-R antagonism on the expression of CGRP in the formalin test should be further elucidated with regard to the time scale.

BBG in the micromolar range was previously shown to inhibit voltage-dependent sodium channels in vitro (Jo and Bean 2011), and it might, therefore, be possible that this feature of BBG contributes to its effects in our experiments. This is rather unlikely, considering that we applied BBG in a single dose, which has been shown not to reach micromolar levels even after continuous administration in mice (Diaz-Hernandez et al. 2012).

In conclusion, our results suggest that $\mathrm{P} 2 \mathrm{X} 7-\mathrm{Rs}$ have a role in the modulation of trigeminal nociceptive processing. Further investigations of the relations of the trigeminal system and P2X7-R signalling may provide important details concerning trigeminal nociceptive processing and the pathomechanism of headaches.

Acknowledgments This work was supported by EUROHEADPAIN FP7, Project Number: 602633 and the Hungarian Brain Research Program-Grant No. KTIA_13_NAP-A-III/9. Dr. Árpád Párdutz is supported by the Bolyai Scholarship Programme of the Hungarian Academy of Sciences. This research was also supported by the European Union and the State of Hungary, co-financed by the European Social Fund in the framework of TÁMOP-4.2.4.A/2-11/12012-0001 'National Excellence Program' and TÁMOP-4.2.2.A-11/ $1 /$ KONV-2012-0052. We are very grateful to Dr. David Durham for the linguistic correction of the manuscript and to Mrs. Valéria Vékony for excellent technical assistance.

\section{Compliance with ethical standards}

Conflict of interest The authors declare that they have no conflict of interest.

\section{References}

Ando RD, Mehesz B, Gyires K, Illes P, Sperlagh B (2010) A comparative analysis of the activity of ligands acting at $\mathrm{P} 2 \mathrm{X}$ and $\mathrm{P} 2 \mathrm{Y}$ receptor subtypes in models of neuropathic, acute and inflammatory pain. Br J Pharmacol 159:1106-1117. doi:10.1111/ j.1476-5381.2009.00596.x

Borsani E, Albertini R, Labanca M, Lonati C, Rezzani R, Rodella LF (2010) Peripheral purinergic receptor modulation influences the trigeminal ganglia nitroxidergic system in an experimental murine model of inflammatory orofacial pain. J Neurosci Res 88:2715-2726. doi:10.1002/jnr.22420

Burnstock G (2013) Purinergic mechanisms and pain-an update. Eur J Pharmacol 716:24-40. doi:10.1016/j.ejphar.2013.01.078

Burstein R, Yarnitsky D, Goor-Aryeh I, Ransil BJ, Bajwa ZH (2000) An association between migraine and cutaneous allodynia. Ann Neurol 47:614-624

Buzzi MG, Carter WB, Shimizu T, Heath H 3rd, Moskowitz MA (1991) Dihydroergotamine and sumatriptan attenuate levels of CGRP in plasma in rat superior sagittal sinus during electrical stimulation of the trigeminal ganglion. Neuropharmacology 30:1193-1200

Chessell IP, Hatcher JP, Bountra C, Michel AD, Hughes JP, Green P et al (2005) Disruption of the P2X7 purinoceptor gene abolishes chronic inflammatory and neuropathic pain. Pain 114:386-396. doi:10.1016/j.pain.2005.01.002

Clavelou P, Pajot J, Dallel R, Raboisson P (1989) Application of the formalin test to the study of orofacial pain in the rat. Neurosci Lett 103:349-353. doi:10.1016/0304-3940(89)90125-0

D'Amico M, Samengo I, Navarra P, Taglialatela M, Martire M (2010) AMPA- and P2X7-receptor-mediated facilitation of [3H]Daspartate release from nerve terminals isolated from the rat caudal brainstem. Neurochem Int 57:623-628. doi:10.1016/j. neuint.2010.07.009

Diaz-Hernandez JI, Gomez-Villafuertes R, Leon-Otegui M, Hontecillas-Prieto L, Del Puerto A, Trejo JL et al (2012) In vivo P2X7 inhibition reduces amyloid plaques in Alzheimer's disease through GSK3beta and secretases. Neurobiol Aging 33:1816-1828. doi:10.1016/j.neurobiolaging.2011.09.040

Friberg L, Olesen J, Olsen TS, Karle A, Ekman R, Fahrenkrug J (1994) Absence of vasoactive peptide release from brain to cerebral circulation during onset of migraine with aura. Cephalalgia 14:47-54

Goloncser F, Sperlagh B (2014) Effect of genetic deletion and pharmacological antagonism of $\mathrm{P} 2 \mathrm{X} 7$ receptors in a mouse animal model of migraine. J Headache Pain 15:24. doi:10.1186/ 1129-2377-15-24

Greco R, Tassorelli C, Sandrini G, Di Bella P, Buscone S, Nappi G (2008) Role of calcitonin gene-related peptide and substance $P$ in different models of pain. Cephalalgia 28:114-126. doi:10.1111/j. 1468-2982.2007.01468.x

Hansen JM, Hauge AW, Olesen J, Ashina M (2010) Calcitonin generelated peptide triggers migraine-like attacks in patients with migraine with aura. Cephalalgia 30:1179-1186. doi:10.1177/ 0333102410368444

Honore P, Donnelly-Roberts D, Namovic MT, Hsieh G, Zhu CZ, Mikusa JP et al (2006) A-740003 [N-(1-\{[(cyanoimino)(5quinolinylamino) methyl]amino $\}$-2,2-dimethylpropyl)-2-(3,4dimethoxyphenyl)acetamide], a novel and selective P2X7 receptor antagonist, dose-dependently reduces neuropathic pain in the rat. J Pharmacol Exp Ther 319:1376-1385. doi:10.1124/jpet.106. 111559

Ito G, Suekawa Y, Watanabe M, Takahashi K, Inubushi T, Murasaki $\mathrm{K}$ et al (2013) P2X7 receptor in the trigeminal sensory nuclear complex contributes to tactile allodynia/hyperalgesia following trigeminal nerve injury. Eur J Pain 17:185-199. doi:10.1002/j. 1532-2149.2012.00174.x

Itoh K, Chiang CY, Li Z, Lee JC, Dostrovsky JO, Sessle BJ (2011) Central sensitization of nociceptive neurons in rat medullary dorsal horn involves purinergic P2X7 receptors. Neuroscience 192:721-731. doi:10.1016/j.neuroscience.2011.06.083

Jiang LH, Mackenzie AB, North RA, Surprenant A (2000) Brilliant Blue G selectively blocks ATP-gated rat P2X(7) receptors. Mol Pharmacol 58:82-88 
Jo S, Bean BP (2011) Inhibition of neuronal voltage-gated sodium channels by Brilliant Blue G. Mol Pharmacol 80:247-257. doi:10.1124/mol.110.070276

Knyihar-Csillik E, Tajti J, Mohtasham S, Sari G, Vecsei L (1995) Electrical stimulation of the Gasserian ganglion induces structural alterations of calcitonin gene-related peptide-immunoreactive perivascular sensory nerve terminals in the rat cerebral dura mater: a possible model of migraine headache. Neurosci Lett 184:189-192. doi:10.1016/0304-3940(94)11203-U

Knyihar-Csillik E, Tajti J, Samsam M, Sary G, Buzas P, Vecsei L (1998) Depletion of calcitonin gene-related peptide from the caudal trigeminal nucleus of the rat after electrical stimulation of the Gasserian ganglion. Exp Brain Res 118:111-114

Lassen LH, Haderslev PA, Jacobsen VB, Iversen HK, Sperling B, Olesen J (2002) CGRP may play a causative role in migraine. Cephalalgia 22:54-61

Limmroth V, Katsarava Z, Liedert B, Guehring H, Schmitz K, Diener HC, Michel MC (2001) An in vivo rat model to study calcitonin gene related peptide release following activation of the trigeminal vascular system. Pain 92:101-106. doi:10.1016/S03043959(00)00475-9

Markowitz S, Saito K, Moskowitz MA (1987) Neurogenically mediated leakage of plasma protein occurs from blood vessels in dura mater but not brain. J Neurosci 7:4129-4136

Matsuka Y, Neubert JK, Maidment NT, Spigelman I (2001) Concurrent release of ATP and substance $\mathrm{P}$ within guinea pig trigeminal ganglia in vivo. Brain Res 915:248-255. doi:10.1016/ S0006-8993(01)02888-8

McGaraughty S, Chu KL, Namovic MT, Donnelly-Roberts DL, Harris RR, Zhang XF et al (2007) P2X7-related modulation of pathological nociception in rats. Neuroscience 146:1817-1828. doi:10.1016/j.neuroscience.2007.03.035
Porro CA, Cavazzuti M (1993) Spatial and temporal aspects of spinal cord and brainstem activation in the formalin pain model. Prog Neurobiol 41:565-607. doi:10.1016/0301-0082(93)90044-S

Samsam M, Covenas R, Ahangari R, Yajeya J, Narvaez JA, Tramu G (1999) Alterations in neurokinin A-, substance P- and calcitonin gene-related peptide immunoreactivities in the caudal trigeminal nucleus of the rat following electrical stimulation of the trigeminal ganglion. Neurosci Lett 261:179-182. doi:10.1016/ S0304-3940(98)00989-6

Surprenant A, Rassendren F, Kawashima E, North RA, Buell G (1996) The cytolytic P2Z receptor for extracellular ATP identified as a P2X receptor (P2X7). Science 272:735-738

Tajti J, Pardutz A, Vamos E, Tuka B, Kuris A, Bohar Z et al (2011) Migraine is a neuronal disease. J Neural Transm 118:511-524. doi:10.1007/s00702-010-0515-3

Takemura M, Shimada T, Sugiyo S, Nokubi T, Shigenaga Y (2000) Mapping of c-Fos in the trigeminal sensory nucleus following high- and low-intensity afferent stimulation in the rat. Exp Brain Res 130:113-123

Teixeira JM, Oliveira MC, Nociti FH Jr, Clemente-Napimoga JT, Pelegrini-da-Silva A, Parada CA, Tambeli CH (2010) Involvement of temporomandibular joint $\mathrm{P} 2 \mathrm{X} 3$ and $\mathrm{P} 2 \mathrm{X} 2 / 3$ receptors in carrageenan-induced inflammatory hyperalgesia in rats. Eur $\mathrm{J}$ Pharmacol 645:79-85. doi:10.1016/j.ejphar.2010.06.008

Wang LG, Li HM, Li JS (1994) Formalin induced FOS-like immunoreactive neurons in the trigeminal spinal caudal subnucleus project to contralateral parabrachial nucleus in the rat. Brain Res 649:62-70. doi:10.1016/0006-8993(94)91049-9 
II. 


\title{
Effect of Probenecid on the Pain-Related Behaviour and Morphological Markers in Orofacial Formalin Test of the Rat
}

\author{
Annamária Fejes-Szabó ${ }^{1}, Z^{2}$ uzsanna Bohár ${ }^{1,2}$, Gábor Nagy-Grócz ${ }^{1}$, Enikő Vámos ${ }^{1}$, Lilla Tar ${ }^{1}$, \\ Borbála Pődör ${ }^{3}$, János Tajti ${ }^{1}$, József Toldi ${ }^{2,4}$, László Vécsei ${ }^{*}{ }^{* 1,2,}$ and Árpád Párdutz ${ }^{1, \S}$
}

\author{
${ }^{I}$ Department of Neurology, Faculty of Medicine, Albert Szent-Györgyi Clinical Centre, University of Szeged, \\ Semmelweis u. 6., Szeged, H-6725, Hungary \\ ${ }^{2}$ MTA-SZTE Neuroscience Research Group, Semmelweis u. 6., Szeged, H-6725, Hungary \\ ${ }^{3}$ Department of Physiology \& Biophysics, Faculty of Medicine, Dalhousie University, Halifax, Nova Scotia, B3H 1 X5, \\ Canada \\ ${ }^{4}$ Department of Physiology, Anatomy and Neuroscience, University of Szeged, Közép fasor 52., Szeged, H-6726, \\ Hungary
}

\begin{abstract}
Probenecid has been widely used in the treatment of gout, but evidence suggests that it may also have anti-nociceptive effects in different inflammatory and pain conditions. We examined the potential modulatory effects of probenecid on behavioural and morphological markers in the orofacial formalin test of the rat.

One hour after pre-treatment with vehicle or probenecid $(1 \mathrm{mmol} / \mathrm{kg}$ body weight $)$ intraperitoneally, $50 \mu 11.5 \%$ formalin solution or physiological saline was injected subcutaneously into the right whisker pad of rats. The rubbing activity directed to the injected whisker pad was then measured for a period of 45 minutes. Four hours after formalin injection, the caudal part of spinal trigeminal nucleus was removed and subjected to c-Fos and neuronal nitric oxide synthase (nNOS) immunohistochemistry and to interleukin-1 $\beta$ and $\mathrm{NAD}(\mathrm{P}) \mathrm{H}$ :quinone oxidoreductase 1 (NQO1) Western blot.
\end{abstract}

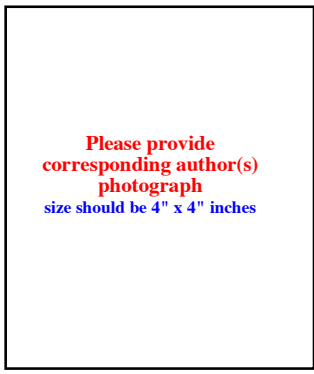

There was a significant decrease in formalin-induced biphasic behavioural response and c-Fos and nNOS immunoreactivity in the rats that were pre-treated with probenecid. However there were no alterations in expression of interleukin-1 $\beta$ or NQO1 after formalin administration.

Our results suggest that probenecid has an anti-nociceptive effect in the trigeminal inflammatory pain model. This effect may be through influencing the release of prostaglandin E2 or desensitizing the transient receptor potential channel subtype A member 1 or the transient receptor potential channel subtype $\mathrm{V}$ member 2 or the effect may be through modulating kynurenic acid levels in the central nervous system. Thus, probenecid might be a potential candidate for the treatment of trigeminal activation related pain conditions.

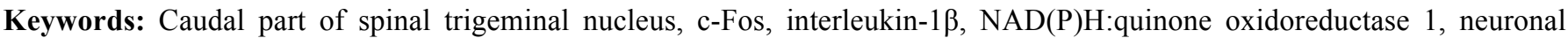
nitric oxide synthase, orofacial formalin test, probenecid.

\section{INTRODUCTION}

Probenecid (PROB, $p$-(di- $n$-propylsulfamyl)benzoic acid) has primarily been used to enhance the renal retention of penicillin [1] and to treat gout, due to its ability to increase the renal excretion of uric acid [2]. Besides its effects on the kidney, research data suggest that PROB may have antiinflammatory and anti-nociceptive properties as well. PROB blocks the multidrug resistance-associated protein 4 (MRP4), an organic anion transporter [3], which is crucial in the release of key inflammatory mediators such as prostaglandin

*Address correspondence to this author at the Department of Neurology, Faculty of Medicine, Albert Szent-Györgyi Clinical Centre, University of Szeged, Semmelweis u. 6, H-6725 Szeged, Hungary; Tel: +36 62 545351; Fax: +36 62 545597; E-mail: vecsei.laszlo@med.u-szeged.hu

${ }^{\S}$ These authors contributed equally to this work.
E1 and prostaglandin E2 (PGE2) [4]. In addition, PROB inhibits the pannexin-1 channel [5], which may be relevant in neuronal inflammatory processes [6]. On the other hand, $\mathrm{PROB}$ is an agonist of the transient receptor potential channel subtype A member 1 (TRPA1) [7] and the transient receptor potential channel subtype V member 2 (TRPV2) [8], which play an important role in pain sensation $[9,10]$. It is also well known, that an agonist-mediated desensitization of these TRPA1 and TRPV2 can be induced [7, 11], leading to analgesia. These results strongly suggest that PROB might be effective in mitigating inflammation and nociception. In contrast, Bang and co-workers raise the possibility that PROB can elicit the pain induced by inflammation through its agonism on TRPV2 [8].

Fundamental research and pharmacological studies investigating the trigeminal inflammation and nociception often use the orofacial formalin test, a reliable model for 
studying somatic pain causing the activation and sensitization of trigeminal system [12], which is crucial in the pathomechanism of headaches such as migraine [13] one of the most common neurological disorders [14]. Formalin solution administered subcutaneously into the upper lip gives rise to a tissue injury, nociception and inflammation [15], activating the primary nociceptors, enhancing the immunoreactivity of c-Fos, a marker of neuronal activation [16] in the caudal part of spinal trigeminal nucleus (TNC) [17] and resulting in an increase in neuronal nitric oxide synthase (nNOS), which is a suggested marker of central sensitization [18]. The induction of formalin causes a biphasic nociceptive behavioural response consisting of rubbing acitivity during an early and shortlasting (3-4 min) first phase of rubbing is followed by a tonic prolonged (20-22 $\mathrm{min}$ ) second phase, the phases being separated by a $9-10 \mathrm{~min}$ quiescent period [19]. The first phase is thought to be due to the direct chemical stimulation of the nociceptors by the formalin solution, while the second phase is a manifestation of the processes induced in the spinal cord during the first phase, together with peripheral inflammation [20]. The inflammatory mediators such as PGE2 and interleukin-1 $\beta$ (IL-1 $\beta$ ) and the oxidative stress may have important role in the formation of changes induced by subcutaneous (s.c.) formalin injection [21-23].

Based on previous experimental results, it has been proposed that PROB, through its different receptorial actions, may have anti-nociceptive effect that can be seen in the orofacial formalin test. Thus the aim of the current study was to investigate whether PROB exerts a modulatory effect in this tonic trigeminal pain model through the examination of behavioural and morphological markers.

\section{MATERIALS AND METHODS}

\section{Animals}

The procedures used in this study followed the guidelines of the eighth edition of the Guide for the Care and Use of Laboratory Animals and the Use of Animals in Research of the International Association for the Study of Pain and the directive of the European Economic Community (86/609/ECC). They were approved by the Committee of Animal Research at the University of Szeged (I-74-12/2012) and the Scientific Ethics Committee for Animal Research of the Protection of Animals Advisory Board (XXIV./352/2012.). Sixty adult male Sprague-Dawley rats weighing 200-250 g were used. The animals were raised and housed under standard laboratory conditions (in an airconditioned, humidity-controlled and ventilated room), with drinking water and regular rat chow available ad libitum on a 12h-12h dark-light cycle. Each rat was used only once.

\section{Drug Administration}

The rats were divided into two groups ( $n=30$ per group). The animals in the Placebo group received only intraperitoneal vehicle solution (physiological saline, $1.5 \mathrm{ml}$ ) as pre-treatment. In the PROB group, the rats were pretreated with an intraperitoneal injection of PROB (Sigma-Aldrich; 1 $\mathrm{mmol} / \mathrm{kg}$ body weight, diluted to $1.5 \mathrm{ml}, \mathrm{pH} 7.4$ ). Both groups of animals were divided further into two subgroups $(n=15): 1$ hour after the PROB or vehicle pre-treatment, half of the animals received a s.c. injection of $50 \mu \mathrm{l} 1.5 \%$ formalin solution $(0.55 \%$ formaldehyde, Sigma-Aldrich) diluted in physiological saline, administered via a 26-gauge needle into the right whisker pad (Placebo-Form and PROBForm), while the other half of the rats were injected with s.c. $50 \mu \mathrm{l}$ physiological saline without formalin (Placebo-Phys and PROB-Phys).

\section{Behavioural Test}

The testing procedures were performed during the light phase (between 8 a.m. and 2 p.m.) in a quiet room. The test box was a $30 \times 30 \times 30 \mathrm{~cm}$ glass terrarium with mirrored walls. For the off-line analysis of rubbing activity directed to the whisker pad, the behaviour of the individually tested rats was recorded with a video camera (Logitech HD Webcam C615) situated $1 \mathrm{~m}$ above the terrarium. One hour after pretreatment with vehicle $(n=20)$ or $\operatorname{PROB}(n=20)$ and after a 10 -min habituation in the test box, the whisker pads of the rats were injected with s.c. formalin ( $n=10$ per subgroup) or physiological saline ( $\mathrm{n}=10$ per subgroup) and the animals were replaced immediately back in the chamber for $45 \mathrm{~min}$. The rats did not receive any food or water during the observation period. The test box was cleaned and decontaminated after each animal. An observer blind to the experimental procedures analysed the recorded videos. The 45-min recording period was divided into $15 \times 3$-min blocks and the total time (number of seconds) spent on rubbing the injected area with the ipsilateral fore- or hindpaw was measured in each block and defined as the nociceptive score for that block. Earlier literature findings led us to use the grooming activity as control, measured in animals that received s.c. physiological saline [15].

\section{c-Fos and nNOS Immunohistochemistry}

Four hours after the formalin or physiological saline injection, the rats $(n=5-9$ per subgroup) were perfused transcardially with $100 \mathrm{ml}$ phosphate-buffered saline (PBS), followed by $500 \mathrm{ml} \mathrm{4 \%}$ paraformaldehyde (Merck) in phosphate buffer under deep chloral hydrate $(0.4 \mathrm{~g} / \mathrm{kg}$ body weight, Sigma-Aldrich) anaesthesia. The medullary segment containing the TNC between +1 and $-5 \mathrm{~mm}$ from the obex was removed, postfixed overnight for immunohistochemistry in the same fixative and cryoprotected (10\% sucrose for $2 \mathrm{~h}$, $20 \%$ sucrose until the blocks sank, and 30\% sucrose overnight). Before sectioning, each segment was marked with a small incision on the ventral and left (contralateral) side of the tissue block, allowing side discrimination during the quantification process. $30-\mu \mathrm{m}$ transverse cryostat sections were cut through the rostrocaudal axis from the beginning of the TNC and were serially collected in wells containing cold PBS. Each well contained every tenth section at $0.3-\mathrm{mm}$ intervals along the rostrocaudal axis (15 levels/sections). The free-floating sections were rinsed in PBS and immersed in $0.3 \% \mathrm{H}_{2} \mathrm{O}_{2}$ (Scharlau Chemie) in PBS for $30 \mathrm{~min}$ to suppress endogenous peroxidase activity. After several rinses in PBS containing 1\% Triton X-100 (PBST; VWR International), sections were incubated at room temperature overnight in PBST containing rabbit anti-rat cFos polyclonal antibody (Santa Cruz Biotechnology, sc-52) 
at a dilution of $1: 2000$ or at $4^{\circ} \mathrm{C}$ for two nights in PBST containing rabbit anti-rat nNOS polyclonal antibody (EuroProxima, B220-1) at a dilution of 1:5,000. The immunohistochemical reaction was visualized by using Vectastain Elite avidin-biotin kits (Vector Laboratories, PK6101). Briefly, the sections were incubated at room temperature for 2 hours in PBST containing goat anti-rabbit biotinylated secondary antibody. After several rinses in PBST, and incubation at room temperature for 2 hours in PBST containing avidin and biotinylated horseradish peroxidase, the sections were stained with 3,3'diaminobenzidine (Sigma-Aldrich) intensified with nickel ammonium sulphate (Scharlau Chemie). The specificity of the immune reactions was checked by omitting the primary antiserum.

The counting of immunoreactive (IR) cells in the TNC was performed by an observer blind to the experimental procedures under the 10x objective of a Nikon Optiphot-2 light microscope in every tenth transverse section in each animal. Before the counting, the location of each section along the rostrocaudal axis and the location of the TNC on each medullary section were determined by means of The Rat Brain in Stereotaxic Coordinates Atlas [24]. The c-Fos neurones with obvious specific nuclear staining and the nNOS-IR neurones with cytoplasmic and dendritic staining and a nucleus were taken into consideration and were counted in the TNC both ipsilaterally and contralaterally to the formalin or physiological saline injection.

\section{IL-1及 and NAD(P)H:Quinone Oxidoreductase 1 (NQO1) Western Blot}

Four hours after the formalin or physiological saline injection, the rats ( $\mathrm{n}=5$ per subgroup) were deeply anaesthetized with chloral hydrate as described above, perfused transcardially with $100 \mathrm{ml}$ ice cold PBS and the ipsi- and contralateral side of the TNC between 0 and $-4 \mathrm{~mm}$ from the obex was removed. Until measurements, the samples were stored at $-80^{\circ} \mathrm{C}$. The TNC segments were sonicated in ice cold lysis buffer containing $50 \mathrm{mM}$ Tris$\mathrm{HCl}, 150 \mathrm{mM} \mathrm{NaCl}, 0.1 \%$ igepal, $0.1 \%$ cholic acid, $2 \mu \mathrm{g} / \mathrm{ml}$ leupeptin, $2 \mathrm{mM}$ phenylmethylsulphonyl fluoride (PMSF), 1 $\mu \mathrm{g} / \mathrm{ml}$ pepstatin, $2 \mathrm{mM}$ EDTA and $0.1 \%$ sodium dodecyl sulphate (SDS) (all chemicals were from Sigma-Aldrich). The lysates were cleared from cellular debris by centrifugation at $12,000 \mathrm{RPM}$ for $10 \mathrm{~min}$ at $4^{\circ} \mathrm{C}$ and supernatants were aliquoted and stored at $-20^{\circ} \mathrm{C}$. Protein concentration was measured according to BCA protein assay method with BCA Protein Assay Kit (Novagen) using bovine serum albumin as a standard. Samples were cooled on ice during the whole procedure. Prior to loading into the gels, each sample was mixed with sample buffer, and denaturated by boiling for $3 \mathrm{~min}$. Equal amounts of protein samples $(20 \mu \mathrm{g} / \mathrm{lane})$ were separated by standard SDS polyacrylamide gel electrophoresis (SDS-PAGE) on $12 \%$ Tris-Glycine gel and electrotransferred onto Amersham Hybond-ECL nitrocellulose membrane $(0.45 \mu \mathrm{m}$ pore size, GE Healthcare). The Page Ruler Prestained Protein Ladder (Fermentas, 10-170 kDa) was used to determine approximate molecular weights. Following the transfer, membranes were blocked for one hour at room temperature in Tris-buffered saline containing Tween 20 (TBST, MP Biomedicals) and
$5 \%$ non fat dry milk powder and then incubated in TBST containing $1 \%$ non fat dry milk and (i) rabbit anti-rat IL-1 $\beta$ (H-153) polyclonal antibody (Santa Cruz Biotechnology, sc7884, dilution: $1: 200$, incubation: overnight at $4^{\circ} \mathrm{C}$ ) or (ii) rabbit anti-rat NQO1 (H-90) polyclonal antibody (Santa Cruz Biotechnology, sc-25591, dilution: 1:200, incubation: overnight at $4^{\circ} \mathrm{C}$ ) or (iii) mouse anti-rat $\beta$-actin monoclonal antibody (Calbiochem, CP01, dilution: 1:100,000, incubation: overnight at $4^{\circ} \mathrm{C}$ ). Next day after several rinses in TBST, membranes were incubated in TBST containing $1 \%$ non fat dry milk and horseradish peroxidase-conjugated anti-rabbit or anti-mouse secondary antibody (Santa Cruz Biotechnology; sc-2030 and sc-2031) for 2 hours at room temperature. Protein bands were visualized after incubation of membranes with the SuperSignal West Pico Chemiluminescent Substrate (Pierce) using Carestream Kodak BioMax Light film (Kodak).

For densitometric analyses, films were scanned and quantified using Java ImageJ $1.47 \mathrm{v}$ analysis software (National Institutes of Health). The $\beta$-actin was used as sample loading control and normalization protein as well. The results from each animal were further normalized to its own contralateral side.

\section{Statistical Analysis}

In the behavioural study, comparisons were made of the rubbing activity in each block of 3-min in each of the subgroups (Placebo-Form, Placebo-Phys, PROB-Form and PROB-Phys) by using two-way repeated measures ANOVA. Subgroups were used as the between-subject factor and 15 blocks of 3-min as the within-subject factor for the analysis. When Mauchly's test of sphericity proved significant, the Greenhouse-Geisser correction was performed. Pairwise comparisons of subgroup means were based on the estimated marginal means with Sidak adjustment for multiple comparisons.

To compare statistical differences between subgroups in the first and second phases, the two phases were determined on the basis of significant results detected during two-way repeated measures ANOVA described above. The first phase was found to be the first of the 15 blocks, while the second phase was the sum of the fifth to the eleventh blocks. To determine significant differences between subgroups in the first or second phase, we used one-way ANOVA followed by the Tamhane post hoc test.

In terms of the immunoreactivity, the numbers of IR neurones in the various subgroups (Placebo-Form, PlaceboPhys, PROB-Form and PROB-Phys) were compared at each level of $0.3-\mathrm{mm}$ (15 levels) along the rostrocaudal axis by using two-way repeated measures ANOVA. Since there was no significant difference in the number of c-Fos- and nNOSIR neurones between the contralateral sides in the subgroups injected with s.c. formalin and the contra- and ipsilateral sides in the subgroups injected with s.c. physiological saline (data not shown), in the course of the statistical analysis the data obtained from the contralateral sides of the subgroups injected with s.c. formalin were used as controls. The contraand ipsilateral sides of the formalin-injected subgroups were used as the between-subject factor, and the 15 levels along the rostrocaudal axis as the within-subject factor for the 
analysis. When Mauchly's test of sphericity proved significant, the Greenhouse-Geisser correction was performed. Pairwise comparisons of subgroup means were based on the estimated marginal means with Sidak adjustment for multiple comparisons.

Statistical analysis of the Western blot data was carried out using one-way ANOVA followed by the Sidak post hoc test. Since there was no significant difference in band densities between the contralateral sides in the subgroups injected with s.c. formalin and the contra- and ipsilateral sides in the subgroups injected with s.c. physiological saline (data not shown), in the course of the statistical analysis the data obtained from the contralateral sides of the subgroups injected with formalin were used as controls.

All tests were two-sided, and probability levels $p<0.05$ were considered to be statistically significant. Group values are reported as means \pm S.E.M.

Statistical analysis of measurements was carried out with IBM SPSS Statistics, version 20 (IBM Corporation) software.

\section{RESULTS}

\section{Nociceptive Response}

The behavioural pattern observed in the rats is in accordance with previous findings $[12,15,19]$. After the formalin injection, the rats immediately withdrew their heads, often accompanied by vocalization. Following their return to the observation box, the rats started to rub their whisker pad continuously and intensely with the ipsilateral forepaw accompanied often by the contralateral forepaw, and occasionally scraped the perinasal area with the ipsilateral hindpaw after a period of $\sim 20 \mathrm{sec}$. This period, referred to as the first phase, lasted 3-4 min, and was followed by a quiescent period of 9-10 min, separating the first phase from the second phase. The second phase was characterized by less intense, but continuous rubbing of the face, predominantly with the ipsilateral forepaw consorted often by the contralateral forepaw as well. This tonic phase lasted $\sim 20-22 \mathrm{~min}$. In the PROB-Form subgroup, the behavioural pattern in both phases was clearly less pronounced than that in the Placebo-Form subgroup, and such behaviour was not witnessed at all in the Placebo-Phys and PROB-Phys subgroups, where the animals displayed very little rubbing/grooming activity.

The comparison of the 3-min blocks revealed significant differences in rubbing activity between the Placebo-Form and Placebo-Phys subgroups in the first $(* * * p<0.001)$ and fifth to eleventh $(* * p<0.01 ; * * * p<0.001)$ blocks (Fig. 1). The PROB pre-treatment significantly decreased the formalin-induced nociceptive behaviour in each block $(\#$ p $<0.01$; \#\#\# $<0.001$; Fig. 1). However, it should be noted that PROB did not attenuate the effect of formalin completely in the first block, where the time spent on rubbing was still significantly higher than that in the Placebo-Phys subgroup $\left({ }^{*} \mathrm{p}<0.05\right.$; Fig. 1). There was no

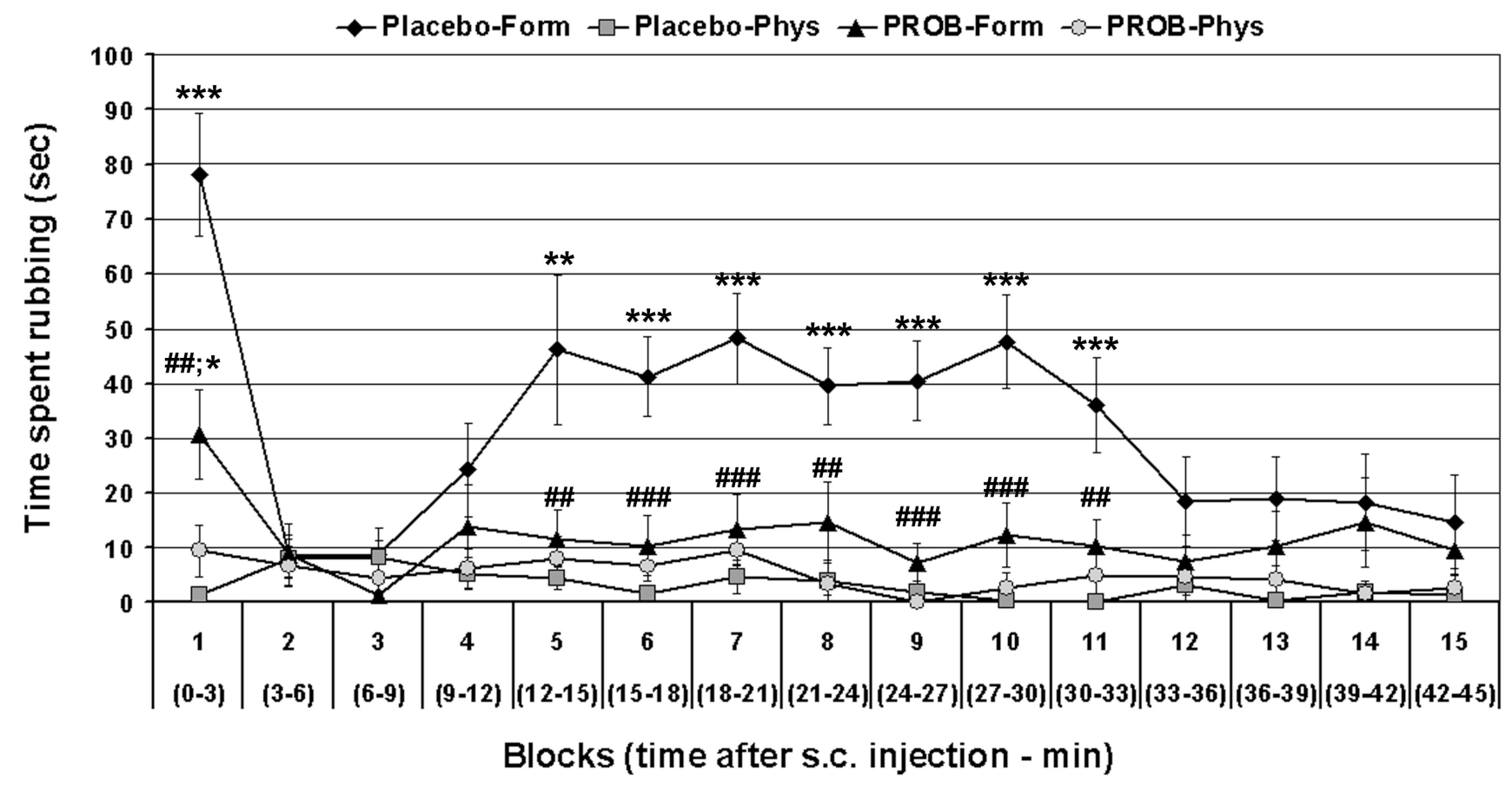

Fig. (1). Diagram showing the time (number of seconds) spent rubbing the injected area with the ipsilateral fore- or hindpaw in each block of $3 \mathrm{~min}$ in all 4 subgroups. In the Placebo-Form subgroup (black diamonds), the two phases of formalin action are clearly distinguishable. The rubbing activity in the first $(* * * p<0.001)$ and in the fifth to eleventh $(* * \mathrm{p}<0.01 ; * * * p<0.001)$ blocks was significantly higher in the Placebo-Form subgroup (black diamonds) than in the Placebo-Phys subgroup (dark-grey squares). The PROB pre-treatment significantly reduced the formalin-induced nociceptive behaviour in each of the above-mentioned blocks $\left({ }^{\#} \mathrm{p}<0.01\right.$; $\left.{ }^{\# \# \# ~}<0.001\right)$ in the PROB-Form subgroup (black triangles) relative to the Placebo-Form subgroup. There was no significant difference between the findings on the PlaceboPhys and PROB-Phys animals in any block. 
significant difference between the Placebo-Phys and PROBPhys animals in any block, nor was between the PROB-Form and PROB-Phys subgroups in any other block apart from the first (Fig. 1).

The face rubbing activity in the Placebo-Form subgroup was significantly higher during both the first $(* * * p<0.001)$ and the second phase $(* * * p<0.001)$ than that in the Placebo-Phys subgroup (Fig. 2). PROB significantly decreased the nociceptive behaviour in both phases $(* * \mathrm{p}<0.01 ; * * * \mathrm{p}<0.001$; Fig. 2), however this effect was not complete in the first phase $(* \mathrm{p}<0.05$; Fig. 2$)$.

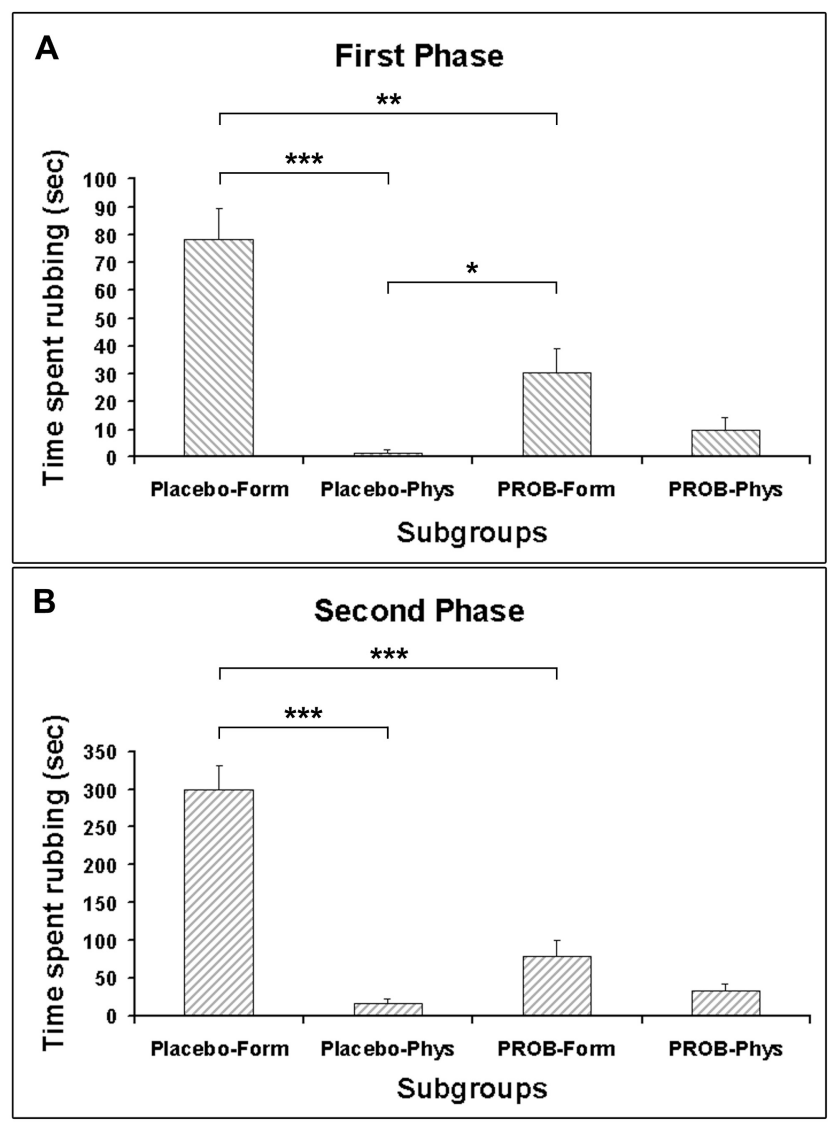

Fig. (2). Diagrams showing the rubbing activity in the first (A) and the second (B) phase in all 4 subgroups. In the Placebo group, the subcutaneous formalin injection (Placebo-Form) induced a significant increase in rubbing activity in both the first and the second phase $(* * * p<0.001)$ as compared with that in the salinetreated animals (Placebo-Phys). In both phases, pre-treatment with PROB had a significant effect on mitigating the formalin-induced increase in the time spent in rubbing $(* * \mathrm{p}<0.01 ; * * * \mathrm{p}<0.001)$ as compared with the Placebo-Form subgroup.

\section{c-Fos in the TNC}

Microscopic examination of the immunostained transverse sections of the TNC revealed c-Fos immunoreactivity in the nuclei of the neurones. In the Placebo-Form subgroup, unilateral s.c. formalin injection produced an increase in the number of c-Fos-IR neurones in the dorsal, superficial area of the transverse sections of the ipsilateral TNC as compared with the non-treated contralateral side (Fig. 3A). This increase was significant at different levels along the rostrocaudal axis (between -0.3 and $-3.3 \mathrm{~mm}$ ), in accordance with the somatotopic representation $(* * \mathrm{p}<0.01 ; * * * \mathrm{p}<0.001$; Fig. 3C).

In the PROB-Form subgroup, the number of c-Fos-IR neurones at the different levels along the rostrocaudal axis also increased in the ipsilateral TNC. This effect was similar, but less pronounced than that in the Placebo-Form subgroup (Fig. 3B), i.e. PROB significantly decreased the formalinrelated activation of the second order trigeminal neurones at several levels of the TNC (between -0.3 and $-2.4 \mathrm{~mm}$; ${ }^{\#} \mathrm{p}<0.05 ; \quad \# \mathrm{p}<0.01 ; \quad \# \# \mathrm{p}<0.001 ;$ Fig. 3C). On the contralateral sides of the TNCs, there were no significant differences either between the subgroups or between the different levels along the rostrocaudal axis (Fig. 3).

\section{nNOS in the TNC}

On the immunostained transverse sections, the nNOS-IR neurones showed cytoplasmic and dendritic staining. The unilateral s.c. formalin injection gave rise to an increase in the number of nNOS-IR neurones in the dorsal, superficial area of the ipsilateral TNC compared to the non-treated contralateral side in the Placebo-Form subgroup (Fig. 4A). Along the rostrocaudal axis, significant increase can be observed at different levels (between -2.1 and -2.7 and between -3.3 and $-3.9 \mathrm{~mm} ;{ }^{*} \mathrm{p}<0.05 ; * * \mathrm{p}<0.01$; Fig. 4C). In the PROB-Form subgroup, there was no difference in the number of nNOS-IR neurones between ipsilateral and contralateral TNC (Fig. 4B), i.e. PROB significantly decreased the number of the second order trigeminal neurones expressing nNOS at several levels of the TNC (between -2.1 and -2.7 and between -3.3 and $-3.9 \mathrm{~mm}$; ${ }^{\#} \mathrm{p}<0.05 ;{ }^{\#} \mathrm{p}<0.01$; Fig. 4C) at the injected side. On the contralateral sides of the TNCs, there were no significant differences either between the subgroups or between the different levels along the rostrocaudal axis (Fig. 4).

\section{IL-1ß and NQO1 in the TNC}

The Western blot analysis of the TNC segments did not show significant difference between the contra- and ipsilateral sides for any of the subgroups either in the case of IL-1 $\beta$ or in the case of NQO1 (data not shown), which means that IL-1 $\beta$ or NQO1 expression did not change four hours after the unilateral s.c. formalin injection and PROB pretreatment did not have any modulatory effect on the expression of IL-1 $\beta$ or of NQO1.

\section{DISCUSSION}

This is the first study showing that PROB produced a pronounced anti-nociceptive behavioural effect in the orofacial formalin test especially in the second phase. PROB exhibited no effect on the baseline activity of the control animals, which received a s.c. saline injection in the whisker pad. In addition to the modulation of the nociceptive behaviour, PROB also mitigated the formalin induced c-Fos and nNOS expression in the TNC - considered markers of activation and sensitization of these neurones [16, 18]. 

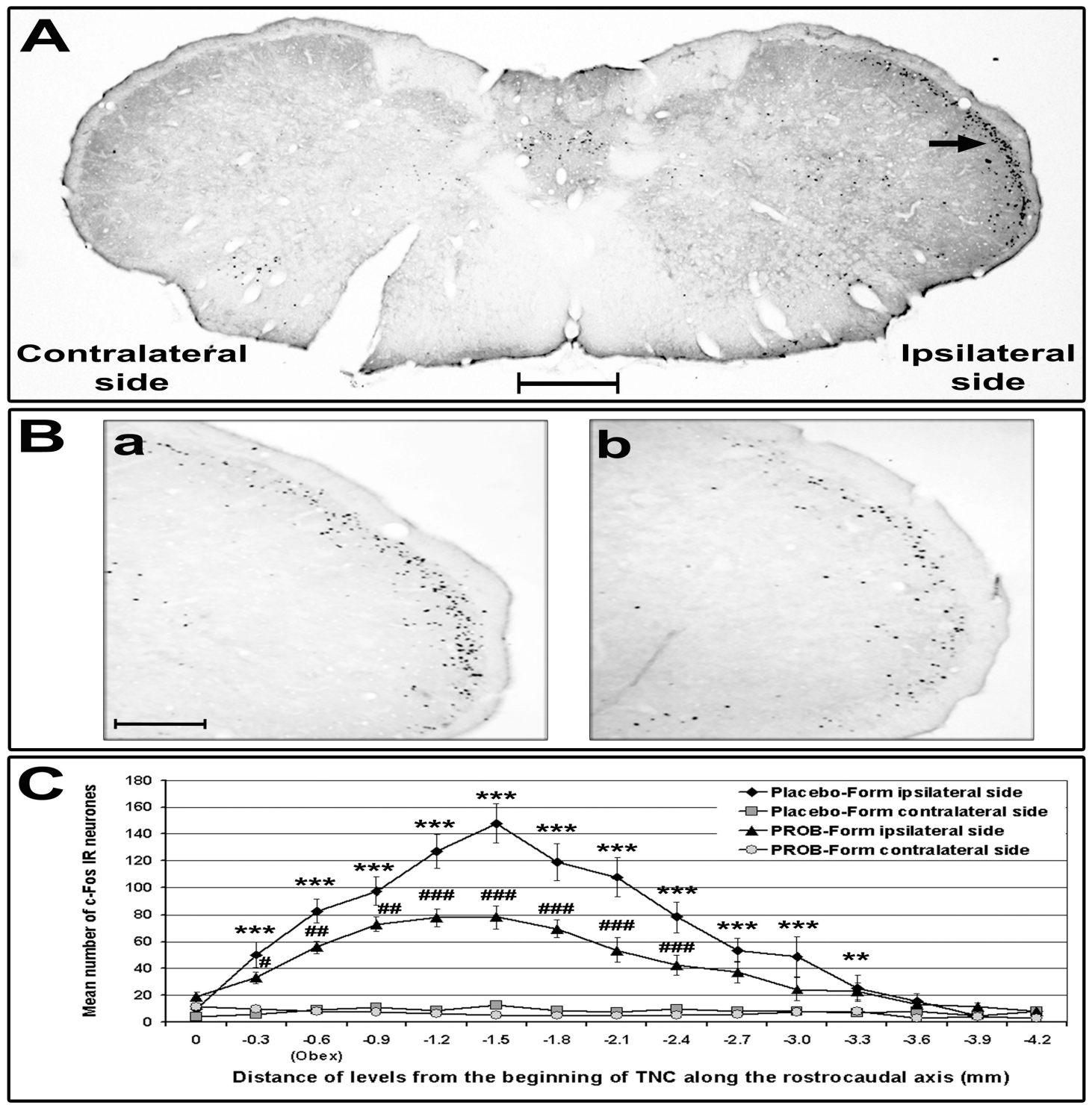

Fig. (3). (A) c-Fos-immunoreactive (IR) neurones (black arrow) in the superficial laminae of a transverse section of medulla containing the caudal part of the spinal trigeminal nucleus (TNC) in the Placebo-Form subgroup. Subcutaneous formalin injection resulted in a higher number of c-Fos-IR neurones on the ipsilateral side than on the contralateral side. Scale bar: $500 \mu \mathrm{m}$. (B) c-Fos-IR neurones on the formalintreated ipsilateral sides of sections in the TNC in the two subgroups. The formalin-induced increase in IR cells was more prominent on the ipsilateral side in the Placebo-Form subgroup (B/a) than in the probenecid (PROB) (B/b)-pretreated animals. Scale bar: $200 \mu \mathrm{m}$. (C) Diagram showing the mean number of c-Fos-IR cells in the superficial laminae of the TNC at different levels along the rostrocaudal axis (mean \pm S.E.M, $n=9$ per subgroup). In the Placebo-Form subgroup, formalin produced a significantly higher number of c-Fos-IR neurones on the formalin-treated side of the TNC as compared with the contralateral side at different levels along the rostrocaudal axis (between -0.3 and -3.3 $\mathrm{mm})$, in accordance with the somatotopic representation $(* * \mathrm{p}<0.01 ; * * * \mathrm{p}<0.001)$. PROB pre-treatment significantly decreased the effect of formalin between -0.3 and $-2.4 \mathrm{~mm}\left({ }^{\#} \mathrm{p}<0.05 ;{ }^{\#} \mathrm{p}<0.01 ;{ }^{\# \#} \mathrm{p}<0.001\right)$.

This anti-nociceptive effect of PROB along with its inhibitory effect on the trigeminal activation and sensitization can be the result of various mechanisms:

PROB inhibits MRP4, an organic anion transporter [3], which releases key inflammatory mediators such as prostaglandin E1 and PGE2 from cells in a time- and ATPdependent manner [4], and these mediators contribute to inflammation and hyperalgesia $[25,26]$. PGE2 released from cultured rat trigeminal ganglion nerve cells [27] can sensitize transient receptor potential channel subtype $\mathrm{V}$ member 1 (TRPV1) [28] (a key factor in the development of sensitization during nociception [29]). Moreover, PGE2 can induce calcitonin gene-related peptide (CGRP) release (a general event after the activation of first order trigeminal neurones [30]) from cultured trigeminal ganglion cells [31]. PGE2 can also act on the trigeminal system at the level of the central nervous system by enhancing the capsaicininduced CGRP release in slice preparations of the TNC [32]. The expression of cyclooxygenase-2 (an inducible enzyme of prostaglandin synthesis [33]) has also been detected in the neurones and glial cells in the trigeminal ganglion [31] and in the neurones of the TNC [34]. In addition, prostaglandin receptors play a relevant role in trigeminal nociception and 

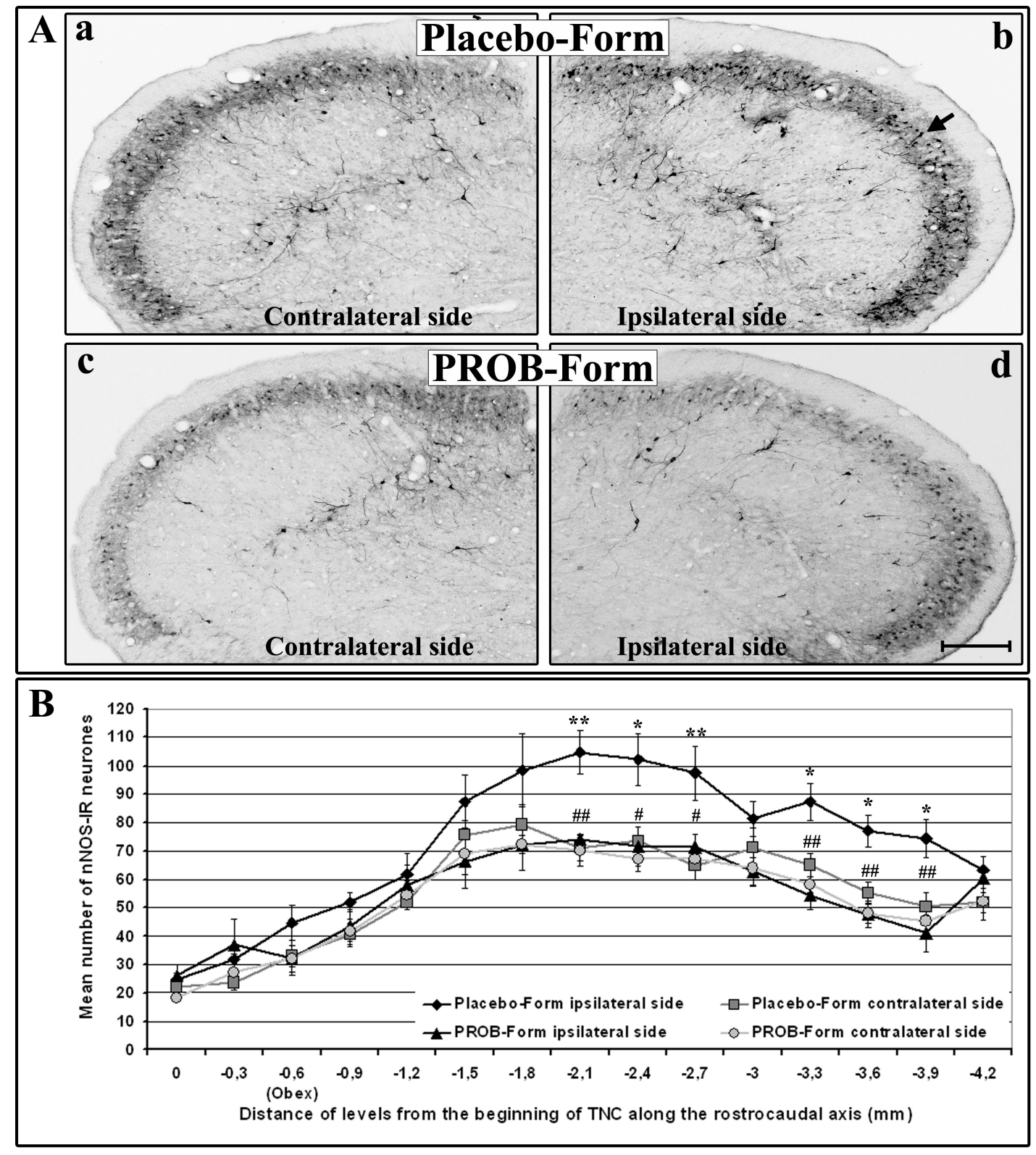

Fig. (4). (A) Immunostained transverse sections of medulla containing the caudal part of the spinal trigeminal nucleus (TNC) from the Placebo-Form and probenecid (PROB)-Form subgroup. Black arrow shows the neuronal nitric-oxide synthase (nNOS)-immunoreactive (IR) neurones in the superficial area of the TNC. On the ipsilateral side (A/b), more nNOS-IR neurones can be observed than on the contralateral side (A/a) in the Placebo-Form subgroup. After formalin injection, there is a lower number of nNOS-IR neurones on the ipsilateral side in the probenecid (PROB)-Form subgroup (A/d) than in the Placebo-Form subgroup (A/b). In these two subgroups, difference between the contralateral sides cannot be observed. Scale bar: $200 \mu \mathrm{m}$. (B) Diagram showing the mean number of nNOS-IR cells in the superficial area of the TNC at different levels along the rostrocaudal axis (mean \pm S.E.M, $n=5$ per subgroup). Formalin produced a significant increase in number of nNOS-IR neurones on the formalin-treated side of the TNC in the Placebo-Form subgroup as compared with the contralateral side at different levels along the rostrocaudal axis (between -2.1 and -2.7 and between -3.3 and $-3.9 \mathrm{~mm} ;{ }^{*} \mathrm{p}<0.05 ; * * \mathrm{p}<0.01$ ). The effect of formalin was significantly decreased by pre-treatment with PROB between -2.1 and -2.7 and between -3.3 and -3.9 mm $\left({ }^{\#} \mathrm{p}<0.05\right.$; $\# \#$ p 0.01). There was no significant difference between the contralateral sides.

in the pathomechanism of trigeminal activation $[35,36]$. They are present in the trigeminal ganglion [37], coexpressed with TRPV1, with a functional interaction between them in the trigeminal system $[38,39]$, and are involved in mediating CGRP release from cultured rat trigeminal neurones [40]. Quantitative autoradiography has revealed that TNC has a moderate density of PGE2 binding sites [41] as well, and the mRNA of all prostaglandin receptors are also present there [37]. Accordingly, a possible mechanism of action of PROB is the inhibition of the release of PGE2, a key player in trigeminal nociception and an important molecule in migraine genesis [42] through the blockade of MRP4.

PROB also inhibits the pannexin-1 channel [5], which may be relevant in inflammation [6] and hence in pain processing. The pannexin-1 channels are mainly expressed in 
the spinal cord [43]; moreover, they are present in the postsynaptic neurones [44] and are crucial in caspase-1 activation, which leads to the production and release of interleukin-1beta (IL-1 $\beta$ ) in neurones, astrocytes [6] and macrophages [45]. The release of IL-1 $\beta$ from trigeminal ganglion satellite cells may contribute to the mechanisms underlying trigeminal inflammatory hyperalgesia [46]. Upregulation of astrocytic IL-1 $\beta$ in the TNC can lead to central sensitization via its receptors present on trigeminal neurones in the same area [47, 48]. The interleukin-1 receptor antagonists were effective in modulation of changes caused by noxious stimulation [26, 49]. Consequently, these results clearly indicate that IL-1 $\beta$ plays a role in trigeminal activation. However the results of our Western blot measurement showed that there were not changes in the expression of IL-1 $\beta$ at the level of TNC four hours after s.c. formalin administration and therefore it is not possible that PROB exerts such effect on this level, but one can not exclude the possibility that PROB affects IL-1 $\beta$ in the periphery, at the inflammation site or in the trigeminal ganglion.

It is important to note that some data suggest that PROB is able to activate some of the transient receptor potential channels, including TRPV2 and TRPA1 [7, 8] which play an important role in pain perception $[9,10]$ and are present in the trigeminal system [50-52]. However, the agonistmediated desensitization of these receptors is also known [7, 11], which can cause an anti-nociceptive effect [53, 54]. PROB can desensitize the TRPA1 [7] and may act similarly on TRPV2 as well, which might account of its antinociceptive properties in the present experiments.

It is a well-known fact that PROB can increase the concentration of kynurenic acid - an endogenous tryptophan metabolite [55] - in the central nervous system [56]. This phenomenon can be caused by inhibition of organic anion transporters by PROB [57, 58], which are involved in the transport of kynurenic acid taking place from brain through the blood-brain barrier [59] or by increasing of concentration of tryptophan in the central nervous system [60]. Experimental data suggest that kynurenic acid can affect nociception [61-64] and elevation in its concentration in the brain can inhibit the trigeminal activation [65, 66], probably due to an antagonistic effect on ionotropic glutamate and $\alpha 7-$ nicotinic acetylcholine receptors [67-69] or due an agonistic action on G-protein-coupled receptor-35 [70], which receptors play important role in pain processing and trigeminal activation [71].

Several lines of evidence confirm the role of oxidative stress in the induction of tissue damage e.g. neuronal activation caused by inflammation in orofacial formalin test $[23,72]$. However, in the present study, we could not support this hypothesis, since the NQO1 expression, a marker of oxidative stress [73, 74], remained unchanged four hours after s.c. formalin injection.

\section{CONCLUSION}

The results of our study provide evidence that pretreatment with PROB can have anti-nociceptive effects and can modulate the changes induced by trigeminal inflammation in both behavioural and morphological markers in the rat orofacial formalin test. PROB may exert this effect through inhibition of the inflammatory processes which could be through blocking the MRP4, which induces the release of key inflammatory mediators such as PGE2 or through desensitization of TRPA1 and TRPV2 or by enhancing concentration of kynurenic acid in the brain. These properties and the relatively good side effect profile can make PROB a possible pharmacological candidate in the treatment of trigeminal activation related pain.

\section{ACKNOWLEDGEMENTS}

This work was supported by the OTKA KI05077, TÁMOP-4.2.2.A-11/1/KONV-2012-0052 and the European Union and the State of Hungary, co-financed by the European Social Fund in the framework of TÁMOP 4.2.4.A/2-11-1-2012-0001 "National Excellence Program", by Hungarian Brain Research Program - Grant No. KTIA_13_NAP-A-III/9 and by the EUROHEADPAIN (FP7-Health 2013-Innovation; Grant no. 602633).

Árpád Párdutz was supported by the Bolyai Scholarship Programme of the Hungarian Academy of Sciences.

The specific contributions of the authors to this research were as follows: Annamária Fejes-Szabó participated in the design and implementation of experiments, collected data for statistical analysis, interpreted the data and wrote the manuscript, Zsuzsanna Bohár, Enikő Vámos and János Tajti participated in the design of the experiments and in the critical revision of the manuscript, Gábor Nagy-Grócz, Lilla Tar and Borbála Pödör participated in the implementation of the experiments, József Toldi participated in the critical revision of the manuscript, László Vécsei participated in the design of the experiments, in the critical revision of the manuscript and in the making final approval of the version to be published, Árpád Párdutz participated in the conception and design of the experiments, the interpretation of the data and the writing and critical revision of the manuscript.

We thank Mrs. Valéria Vékony for the histotechnical assistance, Anna László, M.Sc. for the help in the statistical analysis and David Durham from England for the linguistic correction of the manuscript.

\section{LIST OF ABBREVIATIONS}

$\begin{array}{ll}\text { CGRP } & \text { Calcitonin Gene-Related Peptide } \\ \text { IL-1 } \beta & =\text { Interleukin-1Beta } \\ \text { IR } & \text { Immunoreactive } \\ \text { MRP4 } & \text { Multidrug Resistance-Associated Protein } 4 \\ \text { nNOS } & \text { Neuronal Nitric Oxide Synthase } \\ \text { NQO1 } & \text { NAD(P)H:Quinone Oxidoreductase 1 } \\ \text { PBS } & \text { Phosphate-Buffered Saline } \\ \text { PBST } & \text { Phosphate-Buffered Saline Containing 1\% } \\ \text { PGE2 } & \text { Triton X-100 } \\ \text { PROB } & =\text { Prostaglandin E2 } \\ \text { s.c. } & =\text { Subcutaneous }\end{array}$


$\begin{aligned} \text { TNC }= & \text { Caudal Part of Spinal Trigeminal Nucleus } \\ \text { TRPA1 }= & \text { Transient Receptor Potential Channel Subtype } \\ & \text { A Member } 1\end{aligned}$

\section{CONFLICT OF INTEREST}

The authors confirm that this article content has no conflict of interest.

\section{ACKNOWLEDGEMENTS}

Declared none.

\section{REFERENCES}

[1] Burnell JM, Kirby WM. Effectiveness of a new compound, benemid, in elevating serum penicillin concentrations. J Clin Invest 1951; 30(7): 697-700.

[2] Gutman AB, Yu TF. Benemid (p-di-n-propylsulfamyl)-benzoic acid) as uricosuric agent in chronic gouty arthritis. Trans Assoc Am Physicians 1951; 64: 279-88.

[3] van Aubel RA, Smeets PH, Peters JG, Bindels RJ, Russel FG. The MRP4/ABCC4 gene encodes a novel apical organic anion transporter in human kidney proximal tubules: putative efflux pump for urinary cAMP and cGMP. J Am Soc Nephrol 2002; 13(3): 595-603.

[4] Reid G, Wielinga $\mathrm{P}$, Zelcer $\mathrm{N}$, et al. The human multidrug resistance protein MRP4 functions as a prostaglandin efflux transporter and is inhibited by nonsteroidal antiinflammatory drugs. Proc Natl Acad Sci USA 2003; 100(16): 9244-9.

[5] Silverman W, Locovei S, Dahl G. Probenecid, a gout remedy, inhibits pannexin 1 channels. Am J Physiol Cell Physiol 2008; 295(3): C761-7.

[6] Silverman WR, de Rivero Vaccari JP, Locovei S, et al. The pannexin 1 channel activates the inflammasome in neurons and astrocytes. J Biol Chem 2009; 284(27): 18143-51.

[7] McClenaghan C, Zeng F, Verkuyl JM. TRPA1 agonist activity of probenecid desensitizes channel responses: consequences for screening. Assay Drug Dev Technol 2012; 10(6): 533-41.

[8] Bang S, Kim KY, Yoo S, Lee SH, Hwang SW. Transient receptor potential V2 expressed in sensory neurons is activated by probenecid. Neurosci Lett 2007; 425(2): 120-5.

[9] Shimosato G, Amaya F, Ueda M, Tanaka Y, Decosterd I, Tanaka M. Peripheral inflammation induces up-regulation of TRPV2 expression in rat DRG. Pain 2005; 119(1-3): 225-32.

[10] Bandell M, Story GM, Hwang SW, et al. Noxious cold ion channel TRPA1 is activated by pungent compounds and bradykinin. Neuron 2004; 41(6): 849-57.

[11] Mercado J, Gordon-Shaag A, Zagotta WN, Gordon SE. Ca2+dependent desensitization of TRPV2 channels is mediated by hydrolysis of phosphatidylinositol 4,5-bisphosphate. J Neurosci 2010; 30(40): 13338-47.

[12] Clavelou P, Pajot J, Dallel R, Raboisson P. Application of the formalin test to the study of orofacial pain in the rat. Neurosci Lett 1989; 103(3): 349-53.

[13] D'Andrea G, Leon A. Pathogenesis of migraine: from neurotransmitters to neuromodulators and beyond. Neurol Sci 2010; 31 Suppl 1: S1-7.

[14] Russell MB, Rasmussen BK, Thorvaldsen P, Olesen J. Prevalence and sex-ratio of the subtypes of migraine. Int J Epidemiol 1995; 24(3): 612-8.

[15] Clavelou P, Dallel R, Orliaguet T, Woda A, Raboisson P. The orofacial formalin test in rats: effects of different formalin concentrations. Pain 1995; 62(3): 295-301.

[16] Hunt SP, Pini A, Evan G. Induction of c-fos-like protein in spinal cord neurons following sensory stimulation. Nature 1987; 328(6131): 632-4.
[17] Wang LG, Li HM, Li JS. Formalin induced FOS-like immunoreactive neurons in the trigeminal spinal caudal subnucleus project to contralateral parabrachial nucleus in the rat. Brain Res 1994; 649(1-2): 62-70.

[18] Pardutz A, Krizbai I, Multon S, Vecsei L, Schoenen J. Systemic nitroglycerin increases nNOS levels in rat trigeminal nucleus caudalis. Neuroreport 2000; 11(14): 3071-5.

[19] Raboisson P, Dallel R. The orofacial formalin test. Neurosci Biobehav Rev 2004; 28(2): 219-26.

[20] Tjolsen A, Berge OG, Hunskaar S, Rosland JH, Hole K. The formalin test: an evaluation of the method. Pain 1992; 51(1): 5-17.

[21] Svensson CI, Yaksh TL. The spinal phospholipasecyclooxygenase-prostanoid cascade in nociceptive processing. Annu Rev Pharmacol Toxicol 2002; 42: 553-83.

[22] Watkins LR, Martin D, Ulrich P, Tracey KJ, Maier SF. Evidence for the involvement of spinal cord glia in subcutaneous formalin induced hyperalgesia in the rat. Pain 1997; 71(3): 225-35.

[23] Viggiano E, Monda M, Viggiano A, Aurilio C, De Luca B. Persistent facial pain increases superoxide anion production in the spinal trigeminal nucleus. Mol Cell Biochem 2010; 339(1-2): 14954.

[24] Paxinos G, Watson C. The rat brain in stereotaxic coordinates. 6th ed. Amsterdam: Elsevier 2007.

[25] Ferreira SH, Nakamura M. I - Prostaglandin hyperalgesia, a cAMP/Ca2 + dependent process. Prostaglandins 1979; 18(2): 17990.

[26] Gyires K, Knoll J. Inflammation and writhing syndrome inducing effect of PGE1, PGE2 and the inhibition of these actions. Pol J Pharmacol Pharm 1975; 27(3): 257-64.

[27] Jenkins DW, Sellers LA, Feniuk W, Humphrey PP. Characterization of bradykinin-induced prostaglandin E2 release from cultured rat trigeminal ganglion neurones. Eur J Pharmacol 2003; 469(1-3): 29-36.

[28] Moriyama T, Higashi T, Togashi K, et al. Sensitization of TRPV1 by EP1 and IP reveals peripheral nociceptive mechanism of prostaglandins. Mol Pain 2005; $1: 3$.

[29] Garry MG, Walton LP, Davis MA. Capsaicin-evoked release of immunoreactive calcitonin gene-related peptide from the spinal cord is mediated by nitric oxide but not by cyclic GMP. Brain Res 2000; 861(2): 208-19.

[30] Zhang RX, Mi ZP, Qiao JT. Changes of spinal substance P, calcitonin gene-related peptide, somatostatin, Met-enkephalin and neurotensin in rats in response to formalin-induced pain. Regul Pept 1994; 51(1): 25-32.

[31] Neeb L, Hellen P, Boehnke C, et al. IL-1beta stimulates COX-2 dependent PGE(2) synthesis and CGRP release in rat trigeminal ganglia cells. PLoS One 2011; 6(3): e17360.

[32] Jenkins DW, Langmead CJ, Parsons AA, Strijbos PJ. Regulation of calcitonin gene-related peptide release from rat trigeminal nucleus caudalis slices in vitro. Neurosci Lett 2004; 366(3): 241-4.

[33] Simmons DL, Botting RM, Hla T. Cyclooxygenase isozymes: the biology of prostaglandin synthesis and inhibition. Pharmacol Rev 2004; 56(3): 387-437.

[34] Gao Y, Duan YZ. Increased COX2 in the trigeminal nucleus caudalis is involved in orofacial pain induced by experimental tooth movement. Anat Rec (Hoboken) 2010; 293(3): 485-91.

[35] Kadoi J, Takeda M, Matsumoto S. Prostaglandin E2 potentiates the excitability of small diameter trigeminal root ganglion neurons projecting onto the superficial layer of the cervical dorsal horn in rats. Exp Brain Res 2007; 176(2): 227-36.

[36] Hoffmann J, Neeb L, Israel H, et al. Intracisternal injection of inflammatory soup activates the trigeminal nerve system. Cephalalgia 2009; 29(11): 1212-7.

[37] Myren M, Baun M, Ploug KB, Jansen-Olesen I, Olesen J, Gupta S. Functional and molecular characterization of prostaglandin E2 dilatory receptors in the rat craniovascular system in relevance to migraine. Cephalalgia 2010; 30(9): 1110-22.

[38] Patwardhan AM, Vela J, Farugia J, Vela K, Hargreaves KM. Trigeminal nociceptors express prostaglandin receptors. J Dent Res 2008; 87(3): 262-6.

[39] Price TJ, Patwardhan A, Akopian AN, Hargreaves KM, Flores CM. Modulation of trigeminal sensory neuron activity by the dual cannabinoid-vanilloid agonists anandamide, N-arachidonoyldopamine and arachidonyl-2-chloroethylamide. $\mathrm{Br} \mathrm{J}$ Pharmacol 2004; 141(7): 1118-30. 
[40] Jenkins DW, Feniuk W, Humphrey PP. Characterization of the prostanoid receptor types involved in mediating calcitonin generelated peptide release from cultured rat trigeminal neurones. $\mathrm{Br} \mathrm{J}$ Pharmacol 2001; 134(6): 1296-302.

[41] Matsumura K, Watanabe $\mathrm{Y}$, Imai-Matsumura K, Connolly M, Koyama Y, Onoe H. Mapping of prostaglandin E2 binding sites in rat brain using quantitative autoradiography. Brain Res 1992; 581(2): 292-8.

[42] Antonova M, Wienecke T, Olesen J, Ashina M. Prostaglandin E(2) induces immediate migraine-like attack in migraine patients without aura. Cephalalgia 2012; 32(11): 822-33.

[43] Bruzzone R, Hormuzdi SG, Barbe MT, Herb A, Monyer H. Pannexins, a family of gap junction proteins expressed in brain. Proc Natl Acad Sci USA 2003; 100(23): 13644-9.

[44] Zoidl G, Petrasch-Parwez E, Ray A, et al. Localization of the pannexin 1 protein at postsynaptic sites in the cerebral cortex and hippocampus. Neuroscience 2007; 146(1): 9-16.

[45] Pelegrin P, Surprenant A. Pannexin-1 mediates large pore formation and interleukin-1beta release by the ATP-gated P2X7 receptor. EMBO J 2006; 25(21): 5071-82.

[46] Takeda M, Tanimoto T, Kadoi J, et al. Enhanced excitability of nociceptive trigeminal ganglion neurons by satellite glial cytokine following peripheral inflammation. Pain 2007; 129(1-2): 155-66.

[47] Guo W, Wang H, Watanabe M, et al. Glial-cytokine-neuronal interactions underlying the mechanisms of persistent pain. J Neurosci 2007; 27(22): 6006-18.

[48] Takahashi K, Watanabe M, Suekawa Y, et al. IL-1beta in the trigeminal subnucleus caudalis contributes to extra-territorial allodynia/hyperalgesia following a trigeminal nerve injury. Eur $\mathrm{J}$ Pain 2011; 15(5): 467 e1-14.

[49] Oka T, Aou S, Hori T. Intracerebroventricular injection of interleukin-1 beta enhances nociceptive neuronal responses of the trigeminal nucleus caudalis in rats. Brain Res 1994; 656(2): 23644.

[50] Huang D, Li S, Dhaka A, Story GM, Cao YQ. Expression of the transient receptor potential channels TRPV1, TRPA1 and TRPM8 in mouse trigeminal primary afferent neurons innervating the dura. Mol Pain 2012; 8: 66.

[51] Lee SM, Cho YS, Kim TH, et al. An ultrastructural evidence for the expression of transient receptor potential ankyrin 1 (TRPA1) in astrocytes in the rat trigeminal caudal nucleus. J Chem Neuroanat 2012; 45(1-2): 45-9.

[52] Yamamoto Y, Hatakeyama T, Taniguchi K. Immunohistochemical colocalization of TREK-1, TREK-2 and TRAAK with TRP channels in the trigeminal ganglion cells. Neurosci Lett 2009; 454(2): 129-33.

[53] Akopian AN, Ruparel NB, Jeske NA, Patwardhan A, Hargreaves KM. Role of ionotropic cannabinoid receptors in peripheral antinociception and antihyperalgesia. Trends Pharmacol Sci 2009; 30(2): 79-84.

[54] Materazzi S, Benemei S, Fusi C, et al. Parthenolide inhibits nociception and neurogenic vasodilatation in the trigeminovascular system by targeting the TRPA1 channel. Pain 2013; 154(12): 27508.

[55] Leklem JE. Quantitative aspects of tryptophan metabolism in humans and other species: a review. Am J Clin Nutr 1971; 24(6): 659-72.

[56] Vecsei L, Miller J, MacGarvey U, Beal MF. Kynurenine and probenecid inhibit pentylenetetrazol- and NMDLA-induced seizures and increase kynurenic acid concentrations in the brain. Brain Res Bull 1992; 28(2): 233-8.

[57] Perwitasari O, Yan X, Johnson S, et al. Targeting organic anion transporter 3 with probenecid as a novel anti-influenza a virus strategy. Antimicrob Agents Chemother 2013; 57(1): 475-83.

[58] Chiba S, Ikawa $\mathrm{T}$, Takeshita $\mathrm{H}$, et al. Interactions of human organic anion transporter 1 (hOAT1) with substances associated with forensic toxicology. Leg Med 2011; 13(4): 180-5.

[59] Colin-Gonzalez AL, Santamaria A. Probenecid: an emerging tool for neuroprotection. CNS Neurol Disord Drug Targets 2013; 12(7): 1050-65.

[60] van Wijk M, Sebens JB, Korf J. Probenecid-induced increase of 5hydroxytryptamine synthesis in rat brain, as measured by formation of 5-hydroxytryptophan. Psychopharmacology 1979; 60(3): 22935 .

[61] Nasstrom J, Karlsson U, Post C. Antinociceptive actions of different classes of excitatory amino acid receptor antagonists in mice. Eur J Pharmacol 1992; 212(1): 21-9.

[62] Mecs L, Tuboly G, Nagy E, Benedek G, Horvath G. The peripheral antinociceptive effects of endomorphin- 1 and kynurenic acid in the rat inflamed joint model. Anesth Analg 2009; 109(4): 1297-304.

[63] Pardutz A, Fejes A, Bohar Z, Tar L, Toldi J, Vecsei L. Kynurenines and headache. J Neural Transm 2012; 119(2): 285-96.

[64] Vecsei L, Szalardy L, Fulop F, Toldi J. Kynurenines in the CNS: recent advances and new questions. Nat Rev Drug Discov 2013; 12(1): $64-82$

[65] Vamos E, Pardutz A, Varga H, et al. 1-kynurenine combined with probenecid and the novel synthetic kynurenic acid derivative attenuate nitroglycerin-induced nNOS in the rat caudal trigeminal nucleus. Neuropharmacology 2009; 57(4): 425-9.

[66] Vamos E, Fejes A, Koch J, et al. Kynurenate derivative attenuates the nitroglycerin-induced CamKIIalpha and CGRP expression changes. Headache 2010; 50(5): 834-43.

[67] Birch PJ, Grossman CJ, Hayes AG. Kynurenate and FG9041 have both competitive and non-competitive antagonist actions at excitatory amino acid receptors. Eur J Pharmacol 1988; 151(2): 313-5.

[68] Kessler M, Terramani T, Lynch G, Baudry M. A glycine site associated with N-methyl-D-aspartic acid receptors: characterization and identification of a new class of antagonists. $\mathrm{J}$ Neurochem 1989; 52(4): 1319-28.

[69] Pereira EF, Hilmas C, Santos MD, Alkondon M, Maelicke A, Albuquerque EX. Unconventional ligands and modulators of nicotinic receptors. J Neurobiol 2002; 53(4): 479-500.

[70] Wang J, Simonavicius N, Wu X, et al. Kynurenic acid as a ligand for orphan G protein-coupled receptor GPR35. J Biol Chem 2006; 281(31): 22021-8.

[71] Fejes A, Pardutz A, Toldi J, Vecsei L. Kynurenine metabolites and migraine: experimental studies and therapeutic perspectives. Curr Neuropharmacol 2011; 9(2): 376-87.

[72] Yeo JF, Ling SF, Tang N, Ong WY. Antinociceptive effect of CNS peroxynitrite scavenger in a mouse model of orofacial pain. Exp Brain Res 2008; 184(3): 435-8.

[73] Ali S, Diwakar G, Pawa S. Paraquat induces different pulmonary biochemical responses in Wistar rats and Swiss mice. Chem Biol Interact 2000; 125(2): 79-91.

[74] Tomita M, Okuyama T, Katsuyama H, Hidaka K, Otsuki T, Ishikawa $\mathrm{T}$. Gene expression in rat lungs during early response to paraquat-induced oxidative stress. Int J Mol Med 2006; 17(1): 3744. 
III. 


\title{
Evaluation of c-Fos immunoreactivity in the rat brainstem nuclei relevant in migraine pathogenesis after electrical stimulation of the trigeminal ganglion
}

\author{
Zsuzsanna Bohár • Annamária Fejes-Szabó • Lilla Tar • \\ Hedvig Varga · János Tajti · Árpád Párdutz · László Vécsei
}

Received: 8 August 2012/Accepted: 5 January 2013/Published online: 25 January 2013

(C) Springer-Verlag Italia 2013

\begin{abstract}
Migraine is a common neurological condition, causing high disability, but the pathomechanism of the disease is not yet fully understood. Activation of the trigeminovascular system could play a crucial role in the manifestation of the symptoms, but initial step of this activation remains unknown. Functional imaging studies have revealed that certain brainstem areas, referred to as migraine generators, are activated during a migraine attack, including the dorsal raphe, the periaqueductal gray, the locus coeruleus, and the nucleus raphe magnus. However, the studies performed to date have not demonstrated whether this activation is a trigger or a consequence of the migraine attack. With the aim of evaluating the functional relationship between activation of the trigeminal system and migraine generators, we examined the changes in c-Fos immunoreactivity in the above-mentioned nuclei after stimulation of the trigeminal ganglion, an animal model for trigeminovascular activation. The stimulation led to significant increases in the number of c-Fos immunoreactive cells in the nucleus raphe magnus and in the caudal part of the spinal trigeminal nucleus, 2 and $4 \mathrm{~h}$ after the stimulation. Activation of the trigeminal system failed to exhibit
\end{abstract}

Z. Bohár · A. Fejes-Szabó · L. Tar · J. Tajti · Á. Párdutz ·

L. Vécsei $(\square)$

Department of Neurology, Faculty of Medicine, Albert

Szent-Györgyi Clinical Center, University of Szeged,

Semmelweis u. 6, 6725 Szeged, Hungary

e-mail: vecsei.laszlo@med.u-szeged.hu

Z. Bohár · L. Vécsei

Neuroscience Research Group of the Hungarian Academy

of Sciences and University of Szeged, Szeged, Hungary

H. Varga

Institute of Psychology, University of Szeged, Szeged,

Hungary uniform activation of the brain stem nuclei related to migraine. Our results suggest that the activation of the trigeminal system in the rat by electrical stimulation of the trigeminal ganglion leads to the activation of the descending pain modulatory system, but not to the activation of "migraine generator" nuclei. Therefore, the activity pattern seen in functional studies may reflect a unique feature, exclusively present in migraine.

Keywords Migraine generators - C-Fos - Electrical stimulation of the trigeminal ganglion - Rat

\section{Introduction}

Migraine is a neurological disorder, with an unrevealed pathomechanism. Over the years, many theories have emerged as the cause of the disease; vascular, neurogenic, and genetic processes have been proposed, but none of these could provide a reliable explanation of all the events related to this disorder [1].

During a migraine attack, the trigeminovascular system is activated, neuropeptides and inflammatory mediators are released, leading to neurogenic inflammation with vasodilation and plasma extravasation which can activate the trigeminal nociceptors and cause pain [2].

The activation and sensitization of the trigeminal system play a crucial role in migraine pathomechanism. These activation processes affect the caudal part of the spinal trigeminal nucleus (TNC); therefore, numerous studies have examined the activation pattern of this nucleus in different animal models [3], but the very first step, which leads to these phenomena, remains unexplained.

Functional imaging studies have revealed activation of the dorsolateral pons and the dorsal midbrain during a 
migraine attack, this activation persisting even after successful treatment with antimigraine agents [4], which suggests that this brainstem activation is not related to the perception of pain itself. The experimental results tend to support this view since cranial pain elicited by capsaicin did not give rise to brainstem activation [5]. The data suggest that the dorsal midbrain and dorsolateral pons, including the periaqueductal gray (PAG), the dorsal raphe (DR), the nucleus raphe magnus (NRM), and the locus coeruleus (LC), are involved in migraine headache. Since most of the reported functional imaging studies were carried out in the course of a migraine attack, it has not been ascertained whether the activation of this region is a cause or an effect of the attack.

The NRM comprises part of the descending pain modulatory system receiving strong input from the cerebral cortex [6]. Stimulation of the NRM can modify the nociceptive input at the level of the spinal trigeminal nucleus [7], and the activity of the NRM can be modulated by agents effective in migraine therapy, suggesting a crucial role in the pathomechanism of migraine [8].

The DR, one of the largest serotonergic nuclei in the central nervous system, plays an important part in nociceptive processing and antinociception [9]. This nucleus is activated during trigeminal nociception, thus it is possibly involved in the pathomechanism of migraine [10].

The LC is the main source of norepinephrine in the central nervous system, sending projections both to higher brain structures and to the spinal cord dorsal horn [11]. The LC has been suggested to possess a function parallel to that of NRM in pain control [12]. It has strong projection to the TNC [13], with a possible regulatory role, and sends direct projections to intracranial and extracranial vessels, suggesting an involvement in the vascular events of migraine [14].

Electrical stimulation of the PAG in humans resulted in analgesia [15], but chronic implants, mainly situated in the ventrolateral PAG (VLPAG), caused migraine-like symptoms in some cases [16], implying a role in pain modulation and migraine generation. Experimental and clinical findings have revealed that the PAG is a member of the descending pain modulatory system, which receives input from the spinal cord dorsal horn and from higher brain structures [17]. The PAG can be divided into longitudinal columns, each with different functions, suggesting an integrative role in pain processing, emotional and autonomic regulation [17]. The VLPAG is activated by trigeminal pain in animals [18], indicating that this area has as an integrative role in trigeminal sensory modulation.

Electrical stimulation of the trigeminal ganglion causes structural alterations in calcitonin gene-related peptide positive sensory nerve terminals [19], furthermore it leads to the extravasation of albumin from the vessels of the dura mater [20]. These results indicate that electrical stimulation of the trigeminal ganglion could induce chemical and vascular changes similar to those during migraine pain.

It is clear that the above-mentioned nuclei play an important part during a migraine attack, but it is still unknown whether their role is active or passive. The aim of our study was to investigate their pattern of activation and to evaluate possible functional connections between them and the trigeminal system in an experimental trigeminal activation model, electrical stimulation of the trigeminal ganglion.

\section{Materials and methods}

The procedures of this study conformed to the guidelines of the International Association for the Study of Pain and the European Communities Council (86/609/ECC). They were approved by the Ethics Committee of the Faculty of Medicine, University of Szeged, and by the Committee of Animal Research at the University of Szeged (I-74-14-16/ 2008) and the Scientific Ethics Committee for Animal Research of the Protection of Animals Advisory Board (XI./15.1/02384/001/2007).

Adult male Sprague-Dawley rats (weighing between 280 and $340 \mathrm{~g}$ ) were used. The animals were raised and maintained under standard laboratory conditions, with tap water and regular rat chow available ad libitum, on a 12:12 h dark-light cycle.

\section{Stimulation}

Twenty-six rats were divided into two groups. The animals in the first group $(n=13)$ were deeply anaesthetized with chloral hydrate $(400 \mathrm{mg} / \mathrm{kg}$ ) and placed in a stereotaxic instrument (Stoelting Co., Wood Dale, USA). A hole was drilled with a dental drill $3.2-3.4 \mathrm{~mm}$ posterior to and 2.8-3.2 mm laterally from the bregma. A concentric bipolar electrode (FHC Inc., Bowdoin, USA, CBBRE75) was lowered to the right trigeminal ganglion, and maintained in it for $30 \mathrm{~min}$. After surgery, the animals were kept under deep anaesthesia, with additional doses of chloral hydrate, if needed, being given. They were returned back to their cages and were kept warm.

The animals in the second group $(n=13)$ underwent a similar surgical procedure, but were additionally stimulated for $30 \mathrm{~min}$ with square pulses at $10 \mathrm{~Hz}, 0.5 \mathrm{~mA}$, with a pulse duration of $5 \mathrm{~ms}$. The applied stimulation frequency induces depolarization, thus rapid firing of the pseudounipolar neurones of the trigeminal ganglion, and the intensity applied ensures that the area of the stimulation covers the entire trigeminal ganglion [21]. 
The correct placement of the electrode was checked via the twitching of the jaw when the electrode reached the ganglion, and was confirmed during the autopsy.

From both the groups, seven animals were perfused transcardially $2 \mathrm{~h}$ after the placement of the electrode, while six animals were perfused $4 \mathrm{~h}$ after the electrode placement, with $100 \mathrm{~mL}$ cold $0.1 \mathrm{M}$ phosphate buffered saline (PBS), followed by $500 \mathrm{~mL} 4 \%$ paraformaldehyde in $0.1 \mathrm{M}$ phosphate buffer. The brains and cervical spinal cord were removed and postfixed overnight in the same fixative.
Immunohistochemistry

After cryoprotection $30 \mu \mathrm{m}$ thick serial sections were cut $1 \mathrm{~mm}$ rostrally and $5 \mathrm{~mm}$ caudally from the obex, representing the TNC. The series were collected into 18 wells, and the distance between consecutive sections was $540 \mu \mathrm{m}$.

Similarly $30 \mu \mathrm{m}$ thick serial sections were cut from the brainstem, the series started $1.5 \mathrm{~mm}$ rostrally from the obex and 30 series were cut from each animal. The series
Fig. 1 Representative photos of the caudal part of the spinal trigeminal nucleus from a sham animal (a, c) and from a stimulated one

(b, d) approximately $0.5 \mathrm{~mm}$ caudal from the obex. In sham animals, there was no noticeable difference between the control and the electrode side regarding the number of c-Fos IR cells (a) However, in stimulated animals a remarkable increase in the number of c-Fos IR cells could be observed at the stimulated side (d) compared to the control side of the same animal (b) and also compared to the electrode side of sham animals (c). Rectangles on picture a and b indicate the origin of pictures $\mathbf{c}$ and $\mathbf{d}$, both from the sides of animals associated with the electrode. Scale bars indicate $500 \mu \mathrm{m}$ on $\mathbf{a}$ and $\mathbf{b}$, and $100 \mu \mathrm{m}$ on $\mathbf{c}$ and $\mathbf{d}$
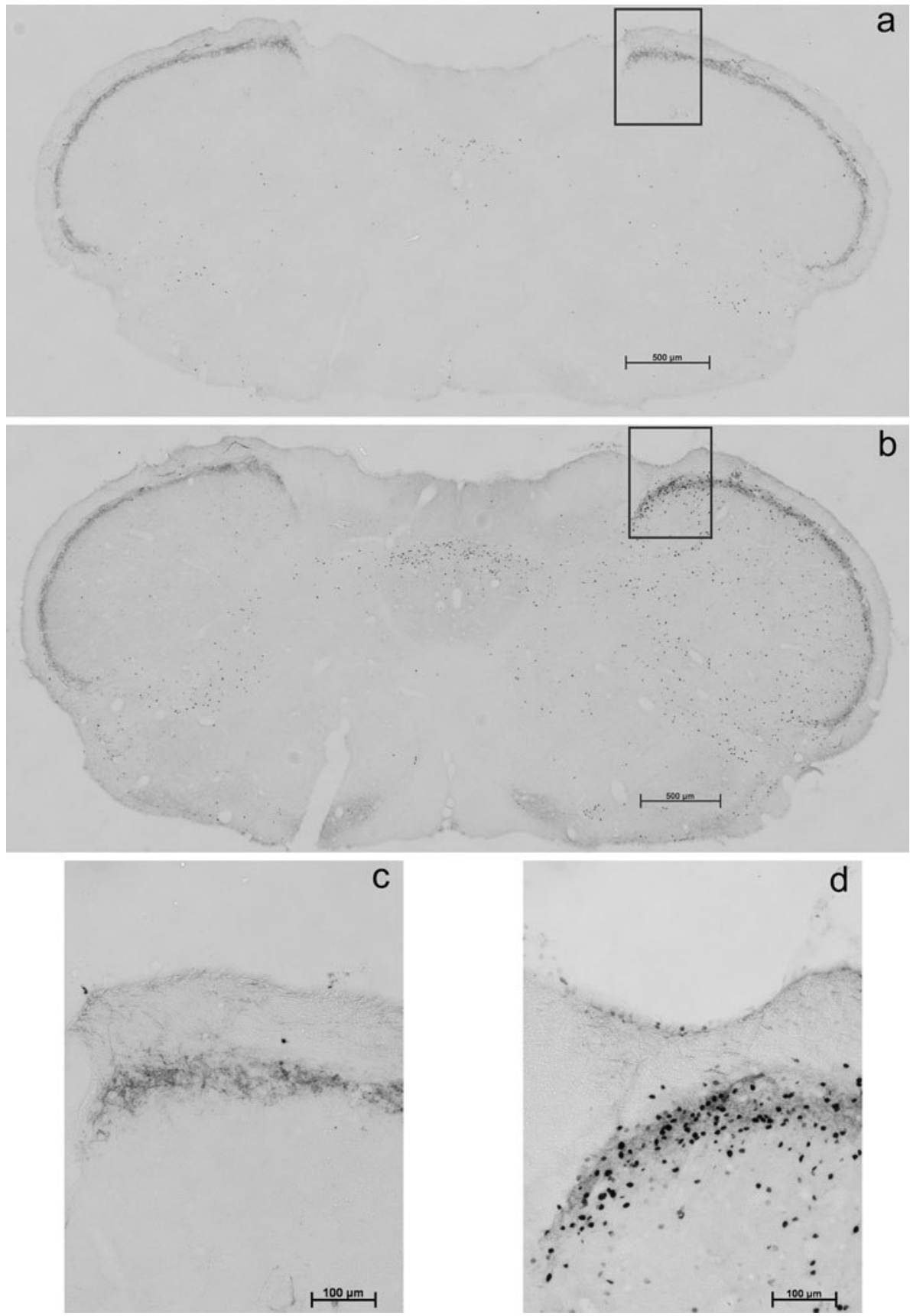
were collected into 10 wells, the distance between consecutive sections therefore being $300 \mu \mathrm{m}$.

The free-floating sections were rinsed in PBS and immersed in methanol containing $0.3 \% \mathrm{H}_{2} \mathrm{O}_{2}$ for 30 min. After several washes in PBS containing $1 \%$ Triton X-100 (PBS-T), the sections were blocked for $1 \mathrm{~h}$ in PBS-T containing $10 \%$ normal goat serum (NGS). Sections from the TNC were incubated for one night at room temperature in PBS-T containing $2 \% \mathrm{NGS}$ and 1:2,000 anti c-Fos primary antibody (Santa Cruz Biotechnology, Santa Cruz, USA, sc-52), while sections from the brainstem were incubated for two nights at $4{ }^{\circ} \mathrm{C}$ in PBS-T containing $2 \%$ NGS and 1:1,000 anti c-Fos primary antibody (Santa Cruz Biotechnology, Santa Cruz, USA, sc-52). The immunohistochemical reaction was visualized using the avidin-biotin kit of Vectastain (Vector Laboratories Inc., Burlingame, USA, PK-6101) and stained with nickel ammonium sulfate-intensified 3,3'-diaminobenzidine for sections of the TNC and 3,3'-diaminobenzidine without intensification for sections of the brainstem. The specificity of the immune reaction was checked by omitting the primary antiserum. On brainstem sections toluidine blue counterstaining was applied to facilitate cell counting.

\section{Cell counting}

The c-Fos-immunopositive cells were counted according to Paxinos and Watson [22] in the TNC, NRM, DR, VLPAG, lateral periaqueductal gray (LPAG), dorsolateral periaqueductal gray (DLPAG), dorsomedial periaqueductal gray (DMPAG), and LC separately. Sections were examined under a Zeiss Axio Imager M2 Upright Microscope (Carl Zeiss MicroImaging, Göttingen, Germany) supplied with an AxioCam MRc camera (Carl Zeiss MicroImaging, Göttingen, Germany). Photographs were taken on $20 \times$ magnification, using the MosaiX program feature of the AxioVision programme. Cells were counted and area of the different nuclei was delineated and measured on one series of sections from the TNC and from the brainstem in each animal. Cell counting was made by an investigator blinded to the stimulation procedure, furthermore stimulated and unstimulated sides of the sections were counted separately.

\section{Statistical analysis}

For the different nuclei, cell counts per side and per series were summed and divided by the corresponding area. In this way, the cell count per $\mu \mathrm{m}^{2}$ was calculated for each side and for each series in each animal.

In case of the TNC counts were averaged by animal and statistical comparison was made using ANOVA (SPSS Statistics 17.0 for Windows, Games-Howell post hoc test).
In case of other paired nuclei of the brainstem, the first step was to determine if there is any difference between the sham and stimulated sides using Student's $t$ test. There was no significant difference in any of the nuclei; therefore, data from separate sides were pooled. These data were than compared using ANOVA (SPSS Statistics 17.0 for Windows, Scheffe post hoc test).

Statistical comparison in the NRM and DR was also conducted by ANOVA (SPSS Statistics 17.0 for Windows, Scheffe post hoc test).

\section{Results}

The surgical procedure and lowering of the electrode to the trigeminal ganglion did not cause significant increase in the number of c-Fos immunoreactive (IR) cells on the ipsilateral side of the TNC neither in the $2 \mathrm{~h}$ nor in the $4 \mathrm{~h}$ survival group (Figs. 1c, 2). On the contralateral (control) side, no changes could be observed (Figs. 1a, 2). After electrical stimulation, a marked increase was observed on the ipsilateral and a slight increase on the contralateral side of the TNC at both survival times (Fig. 1b, d), from which only the changes on the ipsilateral side proved to be significant (Fig. 2). There was no difference in the activation of the TNC between the two stimulated groups.

It is interesting that we found differences in lateralization only in the TNC, in the other nuclei namely the VL, L, DL, DM PAG, and LC, there was no difference between the stimulated and unstimulated sides (data not shown).

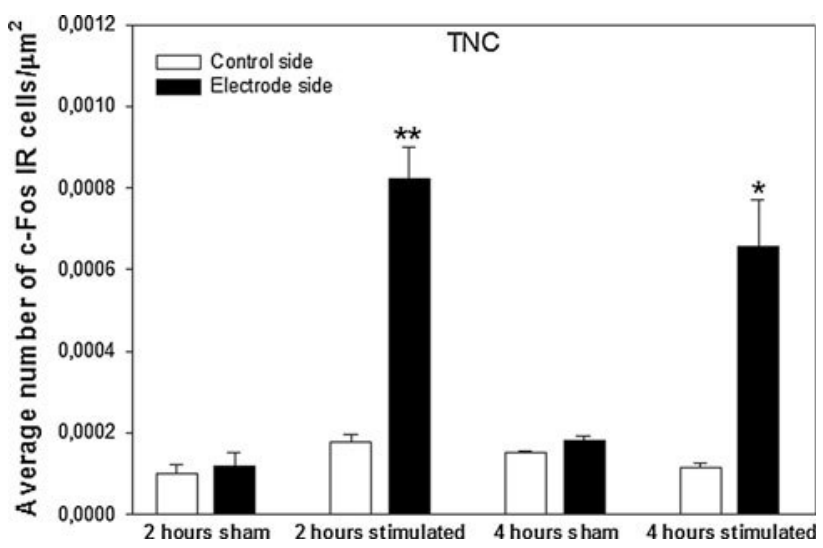

Fig. 2 Diagram showing the average number of c-Fos IR cells per $\mu \mathrm{m}^{2}$ in the caudal part of the spinal trigeminal nucleus (TNC, group means per side + SEM). After sham surgery only a slight nonsignificant increase can be detected, while after electrical stimulation of the trigeminal ganglion a significant increase is present in the TNC both at 2 and $4 \mathrm{~h}$ of survival $(* p<0.05 ; * * p<0.01)$ compared to sham stimulated animals. There is no significant difference between the stimulated sides of the two distinct survival times 
In the NRM, stimulation produced significant $(p<0.05)$ increase in the number of c-Fos IR cells, mainly in the rostral part of the nucleus at both survival times (Fig. 3c, e) compared to the animals with sham stimulation (Fig. 3b, d).

In case of the other nuclei, no statistically significant alteration could be observed between the sham and stimulated groups, although a tendency of increase in the DR and LC could be noticed (Fig. 4).

To evaluate if there is any relationship between the TNC and the NRM regarding the increase in the cell numbers, linear regression analysis was performed (SPSS Statistics 17.0 for Windows). No correlation was found between the two nuclei $\left(R^{2}=0.252 ; F=3.708 ; p=0.80\right)$.
Fig. 3 Representative photos of the nucleus raphe magnus from a stimulated (a, c, e) and a sham animal $(\mathbf{b}, \mathbf{d}) 2(\mathbf{a}, \mathbf{b}, \mathbf{c})$ and $4 \mathrm{~h}$ (d, e) after stimulation approximately $4 \mathrm{~mm}$ rostral from the obex. Rectangle in a indicates the location where $\mathbf{c}$; b, $\mathbf{d}$ and e were taken. Filled arrowheads indicate c-Fos positive cells, while unfilled arrowheads indicate c-Fos negative cells, counterstained with toluidine blue. After $2 \mathrm{~h}$ of survival, c-Fos immunoreactive cells are more numerous in the stimulated group (c). $4 \mathrm{~h}$ after the stimulation the activation of the nucleus raphe magnus is still present (e). Scale bars indicate $1,000 \mu \mathrm{m}$ on $\mathbf{a}$; and $200 \mu \mathrm{m}$ on $\mathbf{b}, \mathbf{c}, \mathbf{d}$ and $\mathbf{e}$

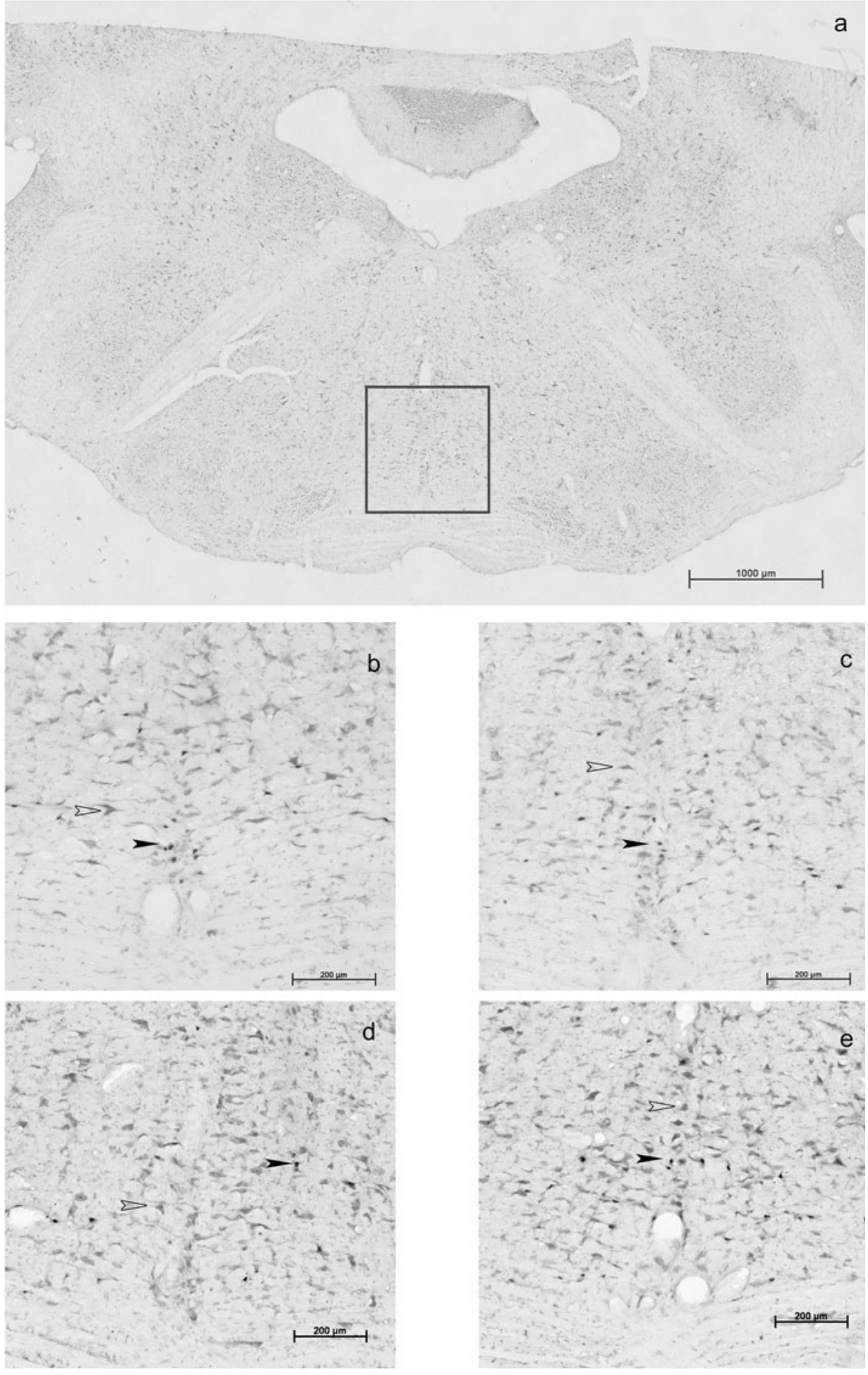




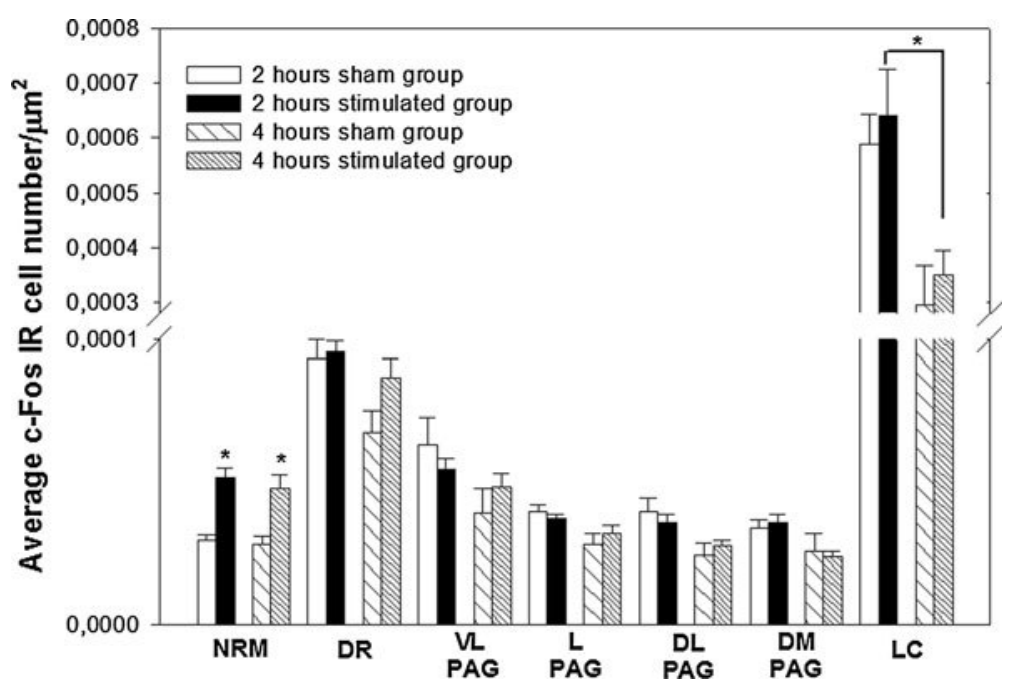

Fig. 4 Diagram showing the average number of c-Fos IR cells per $\mu \mathrm{m}^{2}$ in brainstem nuclei (group means $+\mathrm{SEM}$ ). The highest cell number per area ratio was present in the locus coeruleus, which is not surprising, considering the small extent of this nucleus. Statistically significant alteration between the sham and stimulated group could only be detected in the nucleus raphe magnus $(* p<0.05)$. There is a significant difference $\left({ }^{*} p<0.05\right)$ between the two stimulated groups

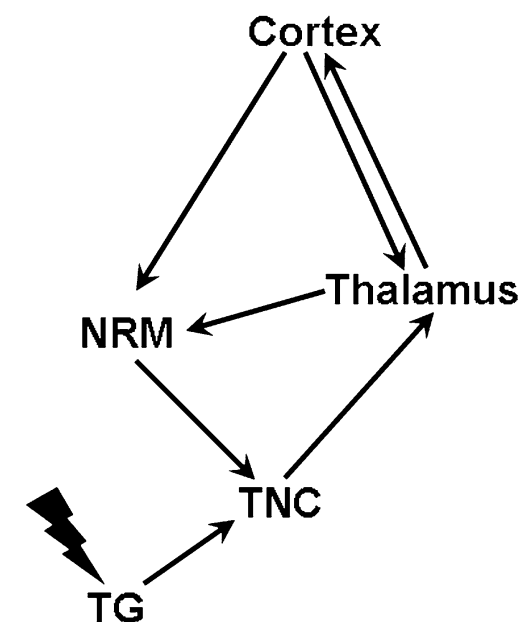

Fig. 5 Schematic route of activation based on our findings. Electrical stimulation activates the secondary trigeminal sensory nuclei in the TNC, the activation travels to the thalamus and the cortex. Activation of the NRM is probably secondarily related to the thalamus

\section{Discussion}

In this study, we first demonstrated that electrical stimulation of the trigeminal ganglion, i.e., activation of the trigeminal system, in the rat does not lead to the uniform activation of "migraine generator nuclei".

Electrical stimulation of the trigeminal ganglion has a direct effect on the primary trigeminal sensory neuron causing alterations in both the peripheral and the central endings. In the periphery, mediators from the nerve in case of the locus coeruleus, but no such difference could be observed in the other nuclei. In the nucleus raphe magnus the stimulation increased the number of c-Fos IR cells. $L C$ locus coeruleus, $D R$ dorsal raphe, $N R M$ nucleus raphe magnus, $V L P A G$ ventrolateral periaqueductal gray, $L P A G$ lateral periaqueductal gray, $D L P A G$ dorsolateral periaqueductal gray, $D M P A G$ dorsomedial periaqueductal gray

endings around the meningeal vessels are released, which results in plasma protein extravasation and eventually neurogenic inflammation [20]. In the central arm, there is a marked activation of the second-order neurones in the TNC [23]. Our results are in accordance with these previous findings as we found marked increase in the number of c-Fos IR cells in the ipsilateral TNC both 2 and $4 \mathrm{~h}$ after stimulation. This pronounced increase may arise directly from the electrical stimulation or is a secondary phenomenon originating from the periphery due to the triggered dural extravasation and inflammation [20]. The activation of second-order neurones is followed by activation of the third-order thalamic and cortical neurones [24].

We observed a significant increase in neuronal activity in the NRM in the $2 \mathrm{~h}$ survival group, which persisted even $4 \mathrm{~h}$ after the stimulation, however a tendency of decrease could be noticed. Direct TNC projections to the NRM are sparse [25], and the superficial laminae, which are mainly activated in response to electrical stimulation, display even fewer connections to the NRM [26]; accordingly activation of the TNC may not evoke a direct NRM activation. This hypothesis is further supported by the fact that we did not find any correlation between the increased cell activities in the two nuclei neither after 2 nor after $4 \mathrm{~h}$, suggesting that the activation of the NRM may not be a direct consequence of the activation of the TNC. Although there is no correlation between the activation patterns of these two nuclei, the increase in their activity is present simultaneously at both 2 and $4 \mathrm{~h}$ after stimulation. 
After intracisternal administration of capsaicin, there is an elevation in the number of c-Fos immunoreactive cells in the NRM [10], suggesting a role in trigeminal nociceptive processing. The NRM is thought to be the main output of the descending pain modulatory system, providing dense innervations to the spinal cord dorsal horn. We assume that activation of the NRM may be a consequence of activation of the descending pain modulatory system. This activation could result from the cortical and thalamic input of the NRM, or it could originate from the PAG [25].

There is no evidence that the TNC projects directly to the DR [27]. The DR forms part of the pain modulatory system in both ascending and descending directions [9], and the DR receives dense afferents from the NRM, but cortical input can also modulate the activity of this nucleus [27]. We did not find significant change in the number of c-Fos IR cells in the DR after electrical stimulation of the TG. Our results suggests that the activation of the trigeminal system does not cause the activation of the DR, and thus in the rat, this nucleus may not participate in the short-term regulation of trigeminal nociceptive processing.

The LC is activated after painful stimuli [10, 28], suggesting an important role in nociceptive processing and modulation, however, activation due to stressful circumstances is also possible [29]. Our results indicate that after electrical stimulation of the TG, activity of the cells in the LC does not change, although it is noticeable that the activity of the LC is already pronounced in the sham group $2 \mathrm{~h}$ after the stimulation, suggesting that the stress and the possible pain caused by the surgical procedure may obscure the changes caused by the electrical stimulation. After $4 \mathrm{~h}$ of survival, the activity level of the LC is decreasing both in the sham and stimulated groups, further supporting our view that the activation of the LC is due to the invasive procedure.

The available information relating to TNC and LC direct inputs is controversial, some authors reporting that the TNC sends only a sparse direct input to the LC [30], and others describing the existence of neuronal input from lamina I to the LC. Our results indicate that the TNC may not have direct input to the LC [31]. It is possible that the contribution of LC to the descending pain modulatory system is via the NRM [32]; however, it is a well-known fact that LC has a descending projection to the spinal cord, which could modulate nociceptive processing directly.

No change in the number of c-Fos immunoreactive nuclei were noticed in any region of the PAG. There is a direct connection between the TNC and the PAG, thus it is surprising that a strong stimulus like we applied caused no alteration in this area. Previous studies indicated an increase in activity of the PAG after nociceptive stimulation [18], although the stimulation parameters and settings differed from those we applied.
If it is assumed that the changes seen in our experiments are secondary, the lack of direct projections from the TNC to most of the above-mentioned nuclei supports this hypothesis. In this conception, the stimulation activates the ascending nociceptive routes to the thalamus and to the cortex, and these structures react to the nociceptive input by activating the descending modulatory system (Fig. 5). After trigeminal stimulation, the examined nuclei failed to exhibit the uniform activation detected during a migraine attack suggesting that this activation pattern of the migraine generator nuclei may be exclusively present in migraine.

Acknowledgments We thank Mrs. Valéria Vékony for her excellent technical assistance. The publication is supported by the European Union and co-funded by the European Social Fund. Project title: "Broadening the knowledge base and supporting the long-term professional sustainability of the Research University Centre of Excellence at the University of Szeged by ensuring the rising generation of excellent scientists". Project number: TÁMOP-4.2.2/B-10/1-20100012. Dr. Árpád Párdutz was supported by the Bolyai Scholarship Programme of the Hungarian Academy of Sciences. Financial support was provided by the Neuroscience Research Group of the Hungarian Academy of Sciences and University of Szeged.

Conflict of interest The authors declare that they have no conflict of interest.

\section{References}

1. Tfelt-Hansen PC, Koehler PJ (2011) One hundred years of migraine research: major clinical and scientific observations from 1910 to 2010. Headache 51(5):752-778. doi:10.1111/j.15264610.2011.01892.x

2. Moskowitz MA (1993) Neurogenic inflammation in the pathophysiology and treatment of migraine. Neurology 43(6 Suppl 3): S16-S20

3. Bergerot A, Holland PR, Akerman S, Bartsch T, Ahn AH, MaassenVanDenBrink A, Reuter U, Tassorelli C, Schoenen J, Mitsikostas DD, van den Maagdenberg AM, Goadsby PJ (2006) Animal models of migraine: looking at the component parts of a complex disorder. Eur J Neurosci 24(6):1517-1534. doi:10.1111/ j.1460-9568.2006.05036.x

4. Weiller C, May A, Limmroth V, Juptner M, Kaube H, Schayck RV, Coenen HH, Diener HC (1995) Brain stem activation in spontaneous human migraine attacks. Nat Med 1(7):658-660

5. May A, Kaube H, Buchel C, Eichten C, Rijntjes M, Juptner M, Weiller C, Diener HC (1998) Experimental cranial pain elicited by capsaicin: a PET study. Pain 74(1):61-66 pii: S0304-3959(97) 00144-9

6. Carlton SM, Leichnetz GR, Young EG, Mayer DJ (1983) Supramedullary afferents of the nucleus raphe magnus in the rat: a study using the transcannula HRP gel and autoradiographic techniques. J Comp Neurol 214(1):43-58. doi:10.1002/cne.902 140105

7. Basbaum AI, Fields HL (1984) Endogenous pain control systems: brainstem spinal pathways and endorphin circuitry. Annu Rev Neurosci 7:309-338. doi:10.1146/annurev.ne.07.030184.001521

8. Ellrich J, Messlinger K, Chiang CY, Hu JW (2001) Modulation of neuronal activity in the nucleus raphe magnus by the 5-HT(1)- 
receptor agonist naratriptan in rat. Pain 90(3):227-231 pii: S030 439590000405X

9. Wang QP, Nakai Y (1994) The dorsal raphe: an important nucleus in pain modulation. Brain Res Bull 34(6):575-585

10. Ter Horst GJ, Meijler WJ, Korf J, Kemper RH (2001) Trigeminal nociception-induced cerebral Fos expression in the conscious rat. Cephalalgia 21(10):963-975 pii: 285

11. Samuels ER, Szabadi E (2008) Functional neuroanatomy of the noradrenergic locus coeruleus: its roles in the regulation of arousal and autonomic function part I: principles of functional organisation. Curr Neuropharmacol 6(3):235-253. doi:10.2174/ 157015908785777229

12. Mokha SS, McMillan JA, Iggo A (1986) Pathways mediating descending control of spinal nociceptive transmission from the nuclei locus coeruleus (LC) and raphe magnus (NRM) in the cat. Exp Brain Res 61(3):597-606

13. Simpson KL, Altman DW, Wang L, Kirifides ML, Lin RC, Waterhouse BD (1997) Lateralization and functional organization of the locus coeruleus projection to the trigeminal somatosensory pathway in rat. J Comp Neurol 385(1):135-147. doi:10.1002/ (SICI) 1096-9861(19970818)385:1<135:AID-CNE8>3.0.CO;2-3

14. Lance JW, Lambert GA, Goadsby PJ, Duckworth JW (1983) Brainstem influences on the cephalic circulation: experimental data from cat and monkey of relevance to the mechanism of migraine. Headache 23(6):258-265

15. Hosobuchi Y, Adams JE, Linchitz R (1977) Pain relief by electrical stimulation of the central gray matter in humans and its reversal by naloxone. Science 197(4299):183-186

16. Raskin NH, Hosobuchi Y, Lamb S (1987) Headache may arise from perturbation of brain. Headache 27(8):416-420

17. Behbehani MM (1995) Functional characteristics of the midbrain periaqueductal gray. Prog Neurobiol 46(6):575-605 pii: 03010082(95)00009-K

18. Hoskin KL, Bulmer DC, Lasalandra M, Jonkman A, Goadsby PJ (2001) Fos expression in the midbrain periaqueductal grey after trigeminovascular stimulation. J Anat 198(Pt 1):29-35

19. Knyihar-Csillik E, Tajti J, Mohtasham S, Sari G, Vecsei L (1995) Electrical stimulation of the Gasserian ganglion induces structural alterations of calcitonin gene-related peptide-immunoreactive perivascular sensory nerve terminals in the rat cerebral dura mater: a possible model of migraine headache. Neurosci Lett 184(3):189-192 pii: 030439409411203U

20. Markowitz S, Saito K, Moskowitz MA (1987) Neurogenically mediated leakage of plasma protein occurs from blood vessels in dura mater but not brain. J Neurosci 7(12):4129-4136

21. Samsam M, Covenas R, Ahangari R, Yajeya J, Narvaez JA, Tramu G (1999) Alterations in neurokinin A-, substance P- and calcitonin gene-related peptide immunoreactivities in the caudal trigeminal nucleus of the rat following electrical stimulation of the trigeminal ganglion. Neurosci Lett 261(3):179-182 pii: S030 4394098009896

22. Paxinos G, Watson C (2007) The Rat Brain in Stereotaxic Coordinates, 6th edn. Academic Press, London

23. Knyihar-Csillik E, Tajti J, Samsam M, Sary G, Slezak S, Vecsei L (1997) Effect of a serotonin agonist (sumatriptan) on the peptidergic innervation of the rat cerebral dura mater and on the expression of $\mathrm{c}$-fos in the caudal trigeminal nucleus in an experimental migraine model. J Neurosci Res 48(5):449-464. doi:10.1002/(SICI)1097-4547(19970601)48:5<449:AID-JNR6> 3.0.CO;2-E

24. Michaud JC, Alonso R, Gueudet C, Fournier M, Calassi R, Breliere JC, Le Fur G, Soubrie P (1998) Effects of SR140333, a selective non-peptide NK1 receptor antagonist, on trigeminothalamic nociceptive pathways in the rat. Fundam Clin Pharmacol 12(1):88-94 pii: S0767398198802338

25. Hermann DM, Luppi PH, Peyron C, Hinckel P, Jouvet M (1997) Afferent projections to the rat nuclei raphe magnus, raphe pallidus and reticularis gigantocellularis pars alpha demonstrated by iontophoretic application of choleratoxin (subunit b). J Chem Neuroanat 13(1):1-21 pii: S0891-0618(97)00019-7

26. Sugiyo S, Takemura M, Dubner R, Ren K (2005) Trigeminal transition zone/rostral ventromedial medulla connections and facilitation of orofacial hyperalgesia after masseter inflammation in rats. J Comp Neurol 493(4):510-523. doi:10.1002/cne.20797

27. Marchand JE, Hagino N (1983) Afferents to the periaqueductal gray in the rat. A horseradish peroxidase study. Neuroscience 9(1):95-106 pii: 0306-4522(83)90049-0

28. Baulmann J, Spitznagel H, Herdegen T, Unger T, Culman J (2000) Tachykinin receptor inhibition and c-Fos expression in the rat brain following formalin-induced pain. Neuroscience 95(3): 813-820 pii: S0306452299004789

29. McDevitt RA, Szot P, Baratta MV, Bland ST, White SS, Maier SF, Neumaier JF (2009) Stress-induced activity in the locus coeruleus is not sensitive to stressor controllability. Brain Res 1285:109-118 pii: S0006-8993(09)01168-8

30. Luppi PH, Aston-Jones G, Akaoka H, Chouvet G, Jouvet M (1995) Afferent projections to the rat locus coeruleus demonstrated by retrograde and anterograde tracing with cholera-toxin B subunit and Phaseolus vulgaris leucoagglutinin. Neuroscience 65(1):119-160 pii: 0306-4522(94)00481-J

31. Craig AD (1992) Spinal and trigeminal lamina I input to the locus coeruleus anterogradely labeled with Phaseolus vulgaris leucoagglutinin (PHA-L) in the cat and the monkey. Brain Res 584(1-2):325-328 pii: 0006-8993(92)90915-V

32. Tanaka M, Matsumoto Y, Murakami T, Hisa Y, Ibata Y (1996) The origins of catecholaminergic innervation in the rostral ventromedial medulla oblongata of the rat. Neurosci Lett 207(1): 53-56 pii: 0304394096124873 